\title{
THE TRANSITIONAL PROTOPLANETARY DISK FREQUENCY AS A FUNCTION OF AGE: DISK EVOLUTION IN THE CORONET CLUSTER, TAURUS, AND OTHER 1-8 Myr OLD REGIONS
}

\author{
Thayne Currie $^{1}$ And Aurora Sicilia-Aguilar ${ }^{2}$ \\ ${ }^{1}$ NASA-Goddard Space Flight Center, Greenbelt, MD, USA \\ ${ }^{2}$ Max-Planck-Institute for Astronomy, Königstuhl 17, 69117 Heidelberg, Germany \\ Received 2010 April 15; accepted 2011 February 22; published 2011 April 11
}

\begin{abstract}
We present Spitzer 3.6-24 $\mu \mathrm{m}$ photometry and spectroscopy for stars in the 1-3 Myr old Coronet Cluster, expanding upon the survey of Sicilia-Aguilar et al. Using sophisticated radiative transfer models, we analyze these new data and those from Sicilia-Aguilar et al. to identify disks with evidence for substantial dust evolution consistent with disk clearing: transitional disks. We then analyze data in Taurus and others young clusters-IC 348, NGC 2362, and $\eta$ Cha-to constrain the transitional disk frequency as a function of time. Our analysis confirms previous results finding evidence for two types of transitional disks - those with inner holes and those that are homologously depleted. The percentage of disks in the transitional phase increases from $\sim 15 \%-20 \%$ at $1-2 \mathrm{Myr}$ to $\geqslant 50 \%$ at 5-8 Myr; the mean transitional disk lifetime is closer to $\sim 1 \mathrm{Myr}$ than $0.1-0.5 \mathrm{Myr}$, consistent with previous studies by Currie et al. and Sicilia-Aguilar et al. In the Coronet Cluster and IC 348, transitional disks are more numerous for very low mass M3-M6 stars than for more massive K5-M2 stars, while Taurus lacks a strong spectral-typedependent frequency. Assuming standard values for the gas-to-dust ratio and other disk properties, the lower limit for the masses of optically thick primordial disks is $M_{\text {disk }} \approx 0.001-0.003 M_{\star}$. We find that single color-color diagrams do not by themselves uniquely identify transitional disks or primordial disks. Full spectral energy distribution modeling is required to accurately assess disk evolution for individual sources and inform statistical estimates of the transitional disk population in large samples using mid-IR colors.
\end{abstract}

Key words: planets and satellites: formation - protoplanetary disks - stars: pre-main sequence

Online-only material: color figures

\section{INTRODUCTION}

Transitional protoplanetary disks identify important stages in planet formation, linking epochs when disks are optically thick primordial protoplanetary disks, rich in gas and containing massive amounts of small dust, and when they are gas-poor/free, optically thin debris disks and thus are in the post gas planetbuilding stage (Currie et al. 2009; Strom et al. 1989). Compared to primordial disks, transitional disks have weaker near-to-midIR emission indicating that they are depleted in warm, micronsized dust within several AU of the host star (e.g., Strom et al. 1989; Calvet et al. 2002). If this dust traces the bulk mass of solids, transitional disks then show evidence for the removal of planet-building material.

Though transitional disks are not primarily identified by their levels of gas, they typically have a lower rate/frequency of stellar accretion (Sicilia-Aguilar et al. 2006a; Muzerolle et al. 2010; Cieza et al. 2010). Transitional disks may also be losing much of their gas from a photoevaporative wind driven by stellar EUV photons (Pascucci \& Sterzik 2009), may lack gas-rich inner disks (Hughes et al. 2008; Salyk et al. 2009), or may have a low density of cold outer disk gas (Hughes et al. 2010). Thus, transitional disks are also plausibly depleted of circumstellar gas responsible for the envelopes of gas/ice giant planets and may be signposts for the last phases of protoplanetary disk evolution and gas giant planet formation.

Spitzer studies have better constrained the morphologies and lifetimes of transitional disks around solar to slightly subsolar-mass stars $\left(M_{\star} \sim 0.5-1.4 M_{\odot}\right.$; see Currie 2010 and Muzerolle 2010 for reviews). Transitional disks exhibit one of two general morphologies: (1) disks with weak or negligible levels of near-to-mid-IR emission indicative of inner dust holes but more strongly emitting, typically optically thick outer disks consistent with an inside-out clearing of dust (Calvet et al. 2005) or (2) disks lacking evidence for inner holes but having weaker emission at all IR wavelengths consistent with a more homologous depletion of dust (Currie et al. 2009). Transitional disks comprise $\lesssim 10 \%-20 \%$ of the disk population in $\approx 1 \mathrm{Myr}$ old clusters (e.g., Muzerolle et al. 2010; Currie \& Kenyon 2009) but are relatively more frequent by $\sim 5 \mathrm{Myr}$, consistent with a transitional disk phase comprising a substantial fraction of the total protoplanetary disk lifetime (e.g., $\sim 1 \mathrm{Myr}$ out of 3-5 Myr; Currie et al. 2009; Sicilia-Aguilar et al. 2009).

The 1-3 Myr old Coronet Cluster (Neuhauser \& Forbrich 2008; Meyer \& Wilking 2009, and references therein) may provide a particularly useful probe of transitional disk properties in the youngest clusters and as a function of stellar mass. SiciliaAguilar et al. (2008) find that transitional disks comprise nearly half of the disk population, much larger than frequencies derived for other clusters of comparable age (e.g., Currie \& Kenyon 2009; Muzerolle et al. 2010). At first glance, these results appear to be in conflict, but Sicilia-Aguilar et al. (2008) note that the members in their sample have systematically low masses (see also López-Martí et al. 2010). Compared to solar and slightly subsolar-mass stars, disks around very low mass stars and brown dwarfs may have very different structures and different dispersal timescales (e.g., Lada et al. 2006; Hartmann et al. 2006). Thus, it is unclear whether the high reported transitional disk frequency in the Coronet Cluster is anomalous or identifies a stellar-massdependent transitional disk frequency. Addressing this issue requires analyzing disks around higher-mass Coronet stars and assessing the transitional disk frequency as a function of stellar mass for many clusters.

In this paper, we investigate the transitional disk population in the Coronet Cluster as a function of spectral type/stellar mass by presenting Spitzer photometry and spectroscopy for additional, 
predominantly solar-mass members. By comparing results for solar-mass and subsolar-mass members, we determine whether the Coronet Cluster disk population shows evidence for a stellarmass-dependent frequency of transitional disks. Section 2 describes our new observations, data reduction, and photometry/ spectroscopy. In Section 3, we model the spectral energy distributions (SEDs) of disk-bearing stars to determine plausible disk evolutionary states following previous methods (e.g., Currie et al. 2009). Finally, in Section 4 we compare these results to those for other clusters to investigate the duration of the transitional disk phase.

\section{DATA}

\subsection{Spitzer IRAC and MIPS Photometry}

To the sample of stars analyzed by Sicilia-Aguilar et al. (2008), we add IRAC and MIPS photometry of other Coronet Cluster members located on the processed IRAC and MIPS mosaics obtained from program IDs 6 (AORs 3650816 and 3664640), 248 (AOR 13469696), and 30784 (AOR 17672960). Image processing and photometry for these stars was performed following methods described in Sicilia-Aguilar et al. (2008). Briefly, the basic calibration data (BCD) frames were mosaiced together using MOPEX using standard input parameters for the overlap correction, pixel interpolation, and outlier rejection. Aperture photometry was performed with APEX using a 3 pixel aperture, a 12-20 pixel background radius, and aperture corrections listed in the IRAC data handbook $(1.112,1.113,1.125$, and 1.218 for the four channels, respectively). For MIPS, we used a 5 pixel aperture with a $8-13$ pixel background annulus, and the aperture correction listed in the MIPS data handbook (1.167).

We select cluster stars from the catalog of Forbrich \& Preibisch (2007), which identifies X-ray bright sources with near-IR/optical counterparts as Coronet Cluster members. We add 32 cluster members from Forbrich \& Preibisch (2007) to the list of stars analyzed in Sicilia-Aguilar et al. (2008). The IRAC and MIPS coverage areas do not completely overlap, and many cluster members lack photometry in two of the four IRAC channels ([3.6] and [5.8] or [4.5] and [8]) or lack MIPS data. Other sources, particularly Class I protostars and bright, higher-mass stars (e.g., R CrA) saturate the IRAC and/or MIPS detector. Table 1 lists the names and coordinates for these targets. Table 2 lists their photometry. Sources with an "FP" prefix for their names were classified as members only by Forbrich \& Preibisch (2007): the numbers following this prefix correspond to the row number in Table 2 of Forbrich \& Preibisch (2007).

To further assess photometric quality, we inspected the processed IRAC and MIPS mosaics and compared the known target positions to the computed target centroids. Most sources lie in regions of low background and are thus uncontaminated by nebulosity. Most sources located in high-background regions are much brighter than the background, which is typically uniform. However, two sources_-TY CrA and HD 176386B — are located in regions of high and highly variable background. In the longer wavelength $5.8 \mu \mathrm{m}$ and $8 \mu \mathrm{m}$ channels, the computed centroid positions are well offset from the apparent star positions, especially for HD 176386B. Figure 1 illustrates this offset for the $5.8 \mu \mathrm{m}$ channel.

To investigate how the mid-IR fluxes from TY CrA and HD 176386 are affected by nebular emission, we recomputed the flux for these sources using much smaller apertures and background annuli, ranging from our default assumptions to a small 2 pixel aperture radius and 2-6 pixel background annulus.
Table 1

Members Listed in Forbrich \& Preibisch (2007) and Included in This Work

\begin{tabular}{|c|c|c|}
\hline Name & R.A. (2000) & Decl. (2000) \\
\hline S CrA & 19:01:08.60 & $-36: 57: 21.3$ \\
\hline F-3 & 19:01:15.86 & $-37: 03: 44.3$ \\
\hline FP-6 & 19:01:19.39 & $-37: 01: 42.0$ \\
\hline FP-8 & $19: 01: 22.40$ & $-37: 00: 55.4$ \\
\hline CrA-134 & 19:01:25.75 & $-36: 59: 19.3$ \\
\hline CrA-135 & $19: 01: 27.15$ & $-36: 59: 8.6$ \\
\hline V709 & $19: 01: 34.84$ & $-37: 00: 56.7$ \\
\hline HD 176386B & 19:01:39.15 & $-36: 53: 29.6$ \\
\hline FP-18 & 19:01:39.34 & $-37: 02: 07.8$ \\
\hline TY CrAabcd & 19:01:40.81 & $-36: 52: 34.0$ \\
\hline IRS2 & 19:01:41.55 & $-36: 58: 31.6$ \\
\hline HBC-677 & 19:01:41.62 & $-36: 59: 53.1$ \\
\hline FP-23 & 19:01:43.12 & $-36: 50: 20.9$ \\
\hline IRS5ab & $19: 01: 48.02$ & $-36: 57: 22.4$ \\
\hline FP-25 & 19:01:48.46 & $-36: 57: 14.5$ \\
\hline IRS6A & 19:01:50.45 & $-36: 56: 38.1$ \\
\hline V710 & 19:01:50.66 & $-36: 58: 09.9$ \\
\hline IRS8 & 19:01:51.11 & $-36: 54: 12.5$ \\
\hline IRS9 & 19:01:52.63 & $-36: 57: 00.2$ \\
\hline $\mathrm{R} \mathrm{CrA}$ & 19:01:53.67 & $-36: 57: 08.3$ \\
\hline IRS7w & 19:01:55.31 & $-36: 57: 22.0$ \\
\hline FP-33 & 19:01:55.61 & $-36: 56: 51.1$ \\
\hline FP-34 & 19:01:55.76 & $-36: 57: 27.7$ \\
\hline FP-35 & 19:01:55.85 & $-36: 52: 04.3$ \\
\hline IRS7e & 19:01:56.39 & $-36: 57: 28.4$ \\
\hline FP-37 & 19:01:57.46 & $-37: 03: 11.9$ \\
\hline FP-38 & 19:01:58.32 & $-37: 00: 27.5$ \\
\hline T CrA & 19:01:58.79 & $-36: 57: 50.1$ \\
\hline V702 & 19:02:01.92 & $-37: 07: 43.0$ \\
\hline B 1858 & 19:02:01.94 & $-36: 54: 00.1$ \\
\hline HBC-679 & 19:02:22.13 & $-36: 55: 41.0$ \\
\hline CrA-159 & 19:02:33.07 & $-36: 58: 21.1$ \\
\hline
\end{tabular}

Notes. Sources with an "FP" prefix followed by a number identify Coronet Cluster members uniquely identified by Forbrich \& Preibisch (2007). The number following "FP" identifies the row number of the source in Table 2 of that paper.

Depending on the choice of aperture radii and background annuli, their fluxes at $5.8 \mu \mathrm{m}$ and $8 \mu \mathrm{m}$ vary by $\sim 50 \%-200 \%$. Therefore, we consider the $5.8 \mu \mathrm{m}$ and $8 \mu \mathrm{m}$ photometry for these sources to be unreliable. Since the nebular background emission increases with wavelength, it also renders fluxes for these sources uncertain at $24-100 \mu \mathrm{m}$.

\subsection{Spitzer IRS Spectroscopy}

To supplement our photometric data, we add spectroscopic data from the Infrared Spectrograph (Houck et al. 2004) spectroscopy for several intermediate-mass members: S CrA, V709, T CrA, IRS7w, and IRS5. The spectra were processed starting from the BCD data and using the spectral extraction tools developed for the FEPS Spitzer science legacy team (Bouwman et al. 2008), based on the SMART software package (Higdon et al. 2004). The spectra were extracted using 6 and 5 pixel wide apertures in the spatial dimension for short- $(7.5-14 \mu \mathrm{m})$ and the long-wavelength $(14-35 \mu \mathrm{m})$ modules, respectively. The background was subtracted using associated pairs of imaged spectra from the two nodded positions along the slit, also eliminating stray light contamination and anomalous dark currents. The long-wavelength modules were not available for $\mathrm{S}$ CrA, and in the cases of IRS7w, IRS5, and T CrA, strong background contamination and/or extended emission makes the 


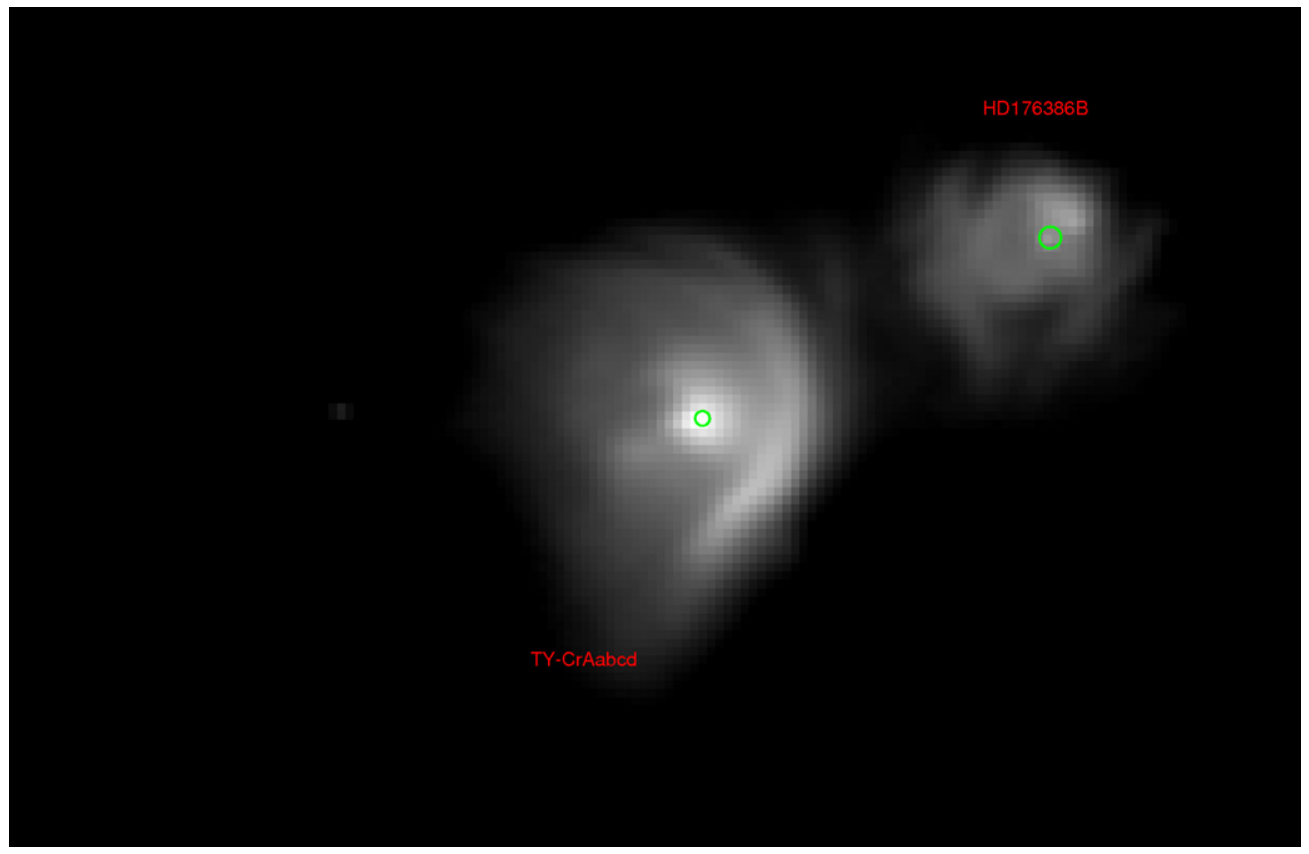

Figure 1. Region of the IRAC [5.8] mosaic showing nebular emission surrounding TY CrA and HD 176386b. The positions of both sources are shown as green circles. Nebular emission contaminates both stars and is responsible for positional offsets between the $2.2-4.5 \mu \mathrm{m}$ centroids and $5.8-8 \mu \mathrm{m}$ centroids. (A color version of this figure is available in the online journal.)

Table 2

Photometry for Members Listed in Forbrich \& Preibisch (2007)

\begin{tabular}{|c|c|c|c|c|c|c|c|c|c|c|c|c|c|c|c|c|}
\hline Name & $J$ & $\sigma(J)$ & $H$ & $\sigma(H)$ & $K_{s}$ & $\sigma\left(K_{s}\right)$ & {$[3.6]$} & $\sigma([3.6])$ & [4.5] & $\sigma([4.5])$ & {$[5.8]$} & $\sigma([5.8])$ & [8] & $\sigma([8])$ & [24] & $\sigma([24])$ \\
\hline$\overline{\mathrm{S} \mathrm{CrA}}$ & 8.19 & 0.02 & 7.05 & 0.024 & 6.10 & 0.02 & 5.060 & 0.005 & 4.386 & 0.005 & 3.699 & 0.005 & 2.969 & 0.004 & & \\
\hline FP-3 & $\ldots$ & $\ldots$ & $\ldots$ & $\ldots$ & $\ldots$ & $\ldots$ & 13.971 & 0.014 & 13.371 & 0.012 & 12.092 & 0.020 & 11.616 & 0.029 & & \\
\hline FP-6 & $\ldots$ & $\ldots$ & $\ldots$ & $\ldots$ & $\ldots$ & $\ldots$ & $\ldots$ & $\ldots$ & $\ldots$ & $\ldots$ & 12.299 & 0.017 & 11.458 & 0.033 & 6.106 & 0.005 \\
\hline FP-8 & $\ldots$ & $\ldots$ & $\ldots$ & $\ldots$ & $\ldots$ & $\ldots$ & $\ldots$ & $\ldots$ & $\ldots$ & $\ldots$ & $\ldots$ & $\ldots$ & 12.697 & 0.092 & $\ldots$ & $\ldots$ \\
\hline CrA-134 & 11.61 & 0.02 & 9.78 & 0.03 & 8.77 & 0.023 & 7.876 & 0.002 & 7.367 & 0.002 & 6.862 & 0.003 & 6.537 & 0.002 & $\ldots$ & $\ldots$ \\
\hline CrA-135 & 10.81 & 0.02 & 10.11 & 0.02 & 9.89 & 0.02 & 9.599 & 0.005 & 9.601 & 0.004 & 9.551 & 0.007 & 9.611 & 0.007 & $\ldots$ & \\
\hline V709 & 8.67 & 0.03 & 7.97 & 0.04 & 7.71 & 0.02 & 7.863 & 0.002 & 7.717 & 0.003 & 7.533 & 0.004 & 7.512 & 0.004 & 6.794 & 0.010 \\
\hline $\mathrm{HD}^{\mathrm{a}}$ & 7.60 & $\ldots$ & 7.49 & 0.09 & 7.14 & 0.08 & 7.837 & 0.003 & 7.781 & 0.003 & 6.243 & 0.003 & 4.706 & 0.003 & $\ldots$ & $\ldots$ \\
\hline \multicolumn{17}{|l|}{176386} \\
\hline FP-18 & 11.34 & 0.03 & 10.66 & 0.03 & 10.37 & 0.03 & 10.110 & 0.005 & 10.097 & 0.005 & 9.979 & 0.007 & 9.927 & 0.010 & 9.364 & 0.104 \\
\hline $\begin{array}{l}\text { TY CrA }{ }^{\mathrm{a}} \\
\text { abcd }\end{array}$ & 7.49 & 0.02 & 6.97 & 0.03 & 6.67 & 0.02 & 6.217 & 0.005 & 6.085 & 0.005 & 4.478 & 0.004 & $\ldots$ & $\ldots$ & $\ldots$ & $\ldots$ \\
\hline IRS2 & 13.75 & 0.02 & 9.74 & 0.02 & 7.07 & 0.02 & 5.181 & 0.004 & 4.281 & 0.003 & $\ldots$ & . & 2.230 & 0.003 & $\ldots$ & $\ldots$ \\
\hline HBC-677 & 10.47 & 0.02 & 9.04 & 0.02 & 8.09 & 0.03 & 7.725 & 0.003 & 7.201 & 0.003 & 6.528 & 0.004 & 5.859 & 0.004 & $\ldots$ & \\
\hline FP-23 & 16.92 & 0.18 & 15.23 & 0.11 & 14.77 & 0.10 & $\ldots$ & $\ldots$ & $\ldots$ & $\ldots$ & $\ldots$ & $\ldots$ & $\ldots$ & $\ldots$ & $\ldots$ & $\ldots$ \\
\hline IRS5ab & 16.9 & $\ldots$ & 13.73 & 0.01 & 10.59 & 0.01 & 7.456 & 0.002 & 6.293 & 0.001 & & $\ldots$ & 3.937 & 0.003 & $\ldots$ & \\
\hline FP-25 & 15.56 & $\ldots$ & 13.14 & $\ldots$ & 12.49 & 0.15 & $\ldots$ & $\ldots$ & 9.115 & 0.003 & & $\cdots$ & 7.017 & 0.003 & $\cdots$ & $\cdots$ \\
\hline IRS6A & 16.39 & 0.20 & 12.14 & .. & 10.31 & .. & 8.480 & 0.003 & 7.687 & 0.004 & $\ldots$ & $\ldots$ & 6.286 & 0.003 & $\ldots$ & $\ldots$ \\
\hline V710 & 15.96 & 0.10 & 11.13 & 0.03 & 7.62 & 0.04 & $\ldots$ & $\ldots$ & 3.659 & 0.003 & $\ldots$ & $\ldots$ & $\ldots$ & $\ldots$ & $\ldots$ & $\ldots$ \\
\hline IRS8 & 14.92 & 0.04 & 12.69 & 0.03 & 11.37 & 0.02 & 9.911 & 0.005 & 9.358 & 0.005 & 8.744 & 0.006 & 7.946 & 0.005 & $\ldots$ & $\ldots$ \\
\hline IRS9 & $\ldots$ & $\ldots$ & $\ldots$ & $\ldots$ & & $\ldots$ & 8.031 & 0.003 & 7.313 & 0.003 & $\ldots$ & $\ldots$ & $\ldots$ & $\ldots$ & $\ldots$ & $\ldots$ \\
\hline $\mathrm{R}$ CrA & 6.952 & 0.021 & 4.955 & 0.024 & 2.910 & 0.318 & $\ldots$ & $\ldots$ & $\ldots$ & $\ldots$ & $\ldots$ & $\ldots$ & $\ldots$ & $\ldots$ & $\cdots$ & $\cdots$ \\
\hline IRS7w & 12.55 & $\ldots$ & 13.25 & 0.32 & 10.76 & $\ldots$ & 8.635 & 0.004 & 7.073 & 0.003 & $\ldots$ & $\ldots$ & $\ldots$ & $\ldots$ & $\ldots$ & $\ldots$ \\
\hline FP-33 & $\ldots$ & $\ldots$ & $\ldots$ & $\ldots$ & $\ldots$ & $\ldots$ & 10.181 & 0.005 & 9.025 & 0.004 & $\ldots$ & $\ldots$ & 6.860 & 0.004 & $\ldots$ & $\ldots$ \\
\hline FP-34 & $\ldots$ & $\ldots$ & $\ldots$ & $\ldots$ & $\ldots$ & $\ldots$ & 9.730 & 0.004 & 8.293 & 0.004 & $\ldots$ & $\ldots$ & $\ldots$ & $\ldots$ & $\ldots$ & $\ldots$ \\
\hline FP-35 & $\ldots$ & $\ldots$ & $\ldots$ & $\ldots$ & $\ldots$ & $\ldots$ & $\ldots$ & $\ldots$ & 13.387 & 0.016 & 11.660 & 0.018 & 10.921 & 0.020 & 7.532 & 0.021 \\
\hline IRS7e & & $\ldots$ & $\ldots$ & $\ldots$ & $\ldots$ & $\ldots$ & 9.417 & 0.004 & $\ldots$ & $\ldots$ & $\ldots$ & $\ldots$ & $\ldots$ & $\ldots$ & $\ldots$ & $\ldots$ \\
\hline FP-37 & 15.58 & 0.07 & 14.02 & 0.04 & 13.19 & 0.04 & 12.320 & 0.007 & 12.264 & 0.006 & 11.925 & 0.089 & 12.086 & 0.037 & & \\
\hline FP-38 & 16.961 & $\ldots$ & 15.275 & 0.139 & 13.704 & 0.060 & 12.426 & 0.007 & 12.102 & 0.008 & 11.508 & 0.017 & 12.008 & 0.050 & $\ldots$ & \\
\hline $\mathrm{T}$ CrA & 8.93 & 0.03 & 7.70 & 0.04 & 6.60 & 0.02 & $\ldots$ & $\ldots$ & 4.819 & 0.005 & 4.217 & 0.005 & $\ldots$ & $\ldots$ & $\cdots$ & $\cdots$ \\
\hline V702 & 8.90 & 0.02 & 8.48 & 0.04 & 8.35 & 0.03 & 8.444 & 0.004 & 8.329 & 0.003 & 8.282 & 0.0048 & 8.280 & 0.003 & 8.122 & 0.034 \\
\hline $\begin{array}{l}\text { B185839.6 } \\
-3658\end{array}$ & $\ldots$ & $\ldots$ & $\ldots$ & $\ldots$ & $\ldots$ & $\ldots$ & 14.099 & 0.019 & 14.766 & 0.054 & $\ldots$ & $\ldots$ & $\ldots$ & $\ldots$ & 8.617 & 0.048 \\
\hline HBC-679 & 10.33 & 0.03 & 9.50 & 0.04 & 9.23 & 0.03 & 9.031 & 0.003 & 8.967 & 0.004 & 8.901 & 0.005 & 8.868 & 0.006 & 8.258 & 0.039 \\
\hline CrA-159 & 10.59 & 0.03 & 9.26 & 0.02 & 8.45 & 0.020 & 7.565 & 0.002 & 7.016 & 0.003 & 6.372 & 0.003 & 5.887 & 0.004 & 3.704 & 0.001 \\
\hline
\end{tabular}

Note. $^{\text {a }}$ (1) Inspection of the IRAC mosaic shows that TY CrAabcd and HD 176386B are contaminated by nebular emission at $5.8 \mu \mathrm{m}$ and $8 \mu \mathrm{m}$. 
Table 3

Provisional Evolutionary States for Coronet Cluster Disks with New Spitzer Photometry Based on 1-24 $\mu \mathrm{m}$ Data

\begin{tabular}{|c|c|c|c|c|c|c|}
\hline ID & ST & ST Ref & $A_{V}$ (best) & $\tau_{\text {mid-IR }}$ & Inner Hole? $\left(R_{\text {sub }}\right)$ & Disk State \\
\hline $\mathrm{S}$ CrA & $\mathrm{G} 5 / \mathrm{K}^{\mathrm{a}}$ & 1 & 2 & Thick & $\mathrm{n}$ & PD \\
\hline CrA-159 & $\mathrm{M} 2^{\mathrm{b}}$ & est. & 3 & Thick & $\mathrm{n}$ & PD \\
\hline IRS2 & $\mathrm{K} 2$ & 2 & $\ldots$ & Thick & $\mathrm{n}$ & PS \\
\hline CrA-134 & K3 & 2 & 11 & Thick & $\mathrm{n}$ & PD \\
\hline CrA-135 & M4 & 2 & 0.5 & $\ldots$ & $\ldots$ & Star \\
\hline V709 & K1 & 7 & 0.5 & Thin/- & $\ldots$ & $\mathrm{Star} / \mathrm{TD}(\mathrm{HD}) / \mathrm{DD} ?$ \\
\hline HD 176396B & K7 & 2 & 0.75 & ? & $\mathrm{n}$ & $?$ \\
\hline FP-18 & M3 & est. & 0.4 & $\ldots$ & $\ldots$ & Star \\
\hline TY CrA & $\mathrm{B} 8 / \mathrm{K} 2$ & 3,4 & 3.5 & $?$ & $\mathrm{n}$ & $?$ \\
\hline HBC-677 & M2 & 2 & 3.9 & Thick & $\mathrm{n}$ & PD \\
\hline IRS5ab & K5 & 2,5 & $\ldots$ & Thick & $\mathrm{n}$ & PS \\
\hline FP-25 & M? & 7 & $\ldots$ & Thick & $\mathrm{n}$ & PS \\
\hline IRS6 & M1 & 2 & 10.5 & Thick & $\mathrm{n}$ & PD \\
\hline V710 & K5-M0 & 5 & $\ldots$ & Thick & $\mathrm{n}$ & PS \\
\hline IRS7 & $?$ & $\ldots$ & $\ldots$ & Thick & $\mathrm{n}$ & PS \\
\hline IRS8 & M2 & est. & 13.5 & Thick & $\mathrm{n}$ & PD \\
\hline $\mathrm{R} \mathrm{CrA}$ & A5 & 7 & $?$ & Thick & $\mathrm{n}$ & PD \\
\hline T CrA & F0 & 6 & 0.3 & Thick & $\mathrm{n}$ & PD \\
\hline V702 & G-5 & 7 & 0.25 & $\ldots$ & $\ldots$ & Star \\
\hline HBC-679 & K2 & 7 & 1.5 & $\ldots$ & $\ldots$ & Star \\
\hline
\end{tabular}

Notes. The disk states are identified as follows: PS: protostar, PD: primordial disk, and TD: transitional disk, star: stellar photosphere (no circumstellar material). The transitional disks are further divided into those with inner holes (IH) and those that are homologously depleted (HD). The disk states here are considered to be provisional since these Coronet Cluster stars lack sensitive far-IR/submillimeter data. The spectral-type references are the following: (1) Carmona et al. 2007, (2) Meyer \& Wilking 2009, (3) Marraco \& Rydgren 1981, (4) Casey et al. 1998, (5) Nisini et al. 2005, (6) Patten 1998, and (7) Forbrich \& Preibisch 2007. "Est" means that the spectral type was estimated by modeling the optical to near-IR SED using the Robitaille et al. (2006) grid and the Currie et al. (2010) effective temperature scale.

${ }^{a}$ The fit to the photosphere for this star is generally poor; the best-fit $A_{V}$ corresponds to a K0 stellar photosphere model. However, the exact value has no bearing on our classification.

b Patten (1998) lists the spectral type for this star as M3-M5. However, we cannot reproduce the observed optical to near-IR SED with an M3-M5 photosphere using either the T. Currie et al. (2011, in preparation) Te scale and dwarf colors or with the Robitaille et al. (2006) grid: all good-fitting models $\left(\chi^{2}-\chi_{\text {best }}^{2}<3\right)$ predict that its photosphere is hotter than $\sim 3800 \mathrm{~K}$ and thus its spectral type is earlier than M0-M2. Since other stars classified as M3-M5 by Patten (1998) are reclassified as M0-M2 by Meyer \& Wilking (2009) based on higher signal-to-noise data, we list this star as an M2 star.

spectral extraction uncertain at $\lambda>14 \mu \mathrm{m}$. Therefore, we only include in the analysis the short-wavelength region for these sources.

\subsection{Ancillary Data}

To compare the stellar and circumstellar properties of all Coronet Cluster members, we add IRAC/MIPS photometry and IRS spectroscopy presented in Sicilia-Aguilar et al. (2008); optical photometry from López-Martí et al. (2005), Torres et al. (2006), and from the SIMBAD Astronomical Database (various sources); near-IR photometry from Two Micron All Sky Survey (2MASS), Skrutskie et al. (2006), and Meyer \& Wilking (2009). Spectral types for most cluster stars with new Spitzer data derive from Meyer \& Wilking (2009) or Nisini et al. (2005). Most stars studied in Sicilia-Aguilar et al. (2008), have spectral types derived from VLT/FLAMES.

We also consider new, higher signal-to-noise VLT/FLAMES spectra of Coronet Cluster members, which yield new spectral types for several low-mass cluster stars-G-1, CrA-4107, CrA4109, CrA-432, CrA-468, CrA-452, G-85, and G-87. These data will be discussed and analyzed in detail in a later contribution (A. Sicilia-Aguilar et al. 2011, in preparation). Briefly, the medium resolution $(R \sim 6000)$ spectra were centered on three bands covering $0.6-0.9 \mu \mathrm{m}\left(\lambda_{c}=0.682,0.773\right.$, and $\left.0.881 \mu \mathrm{m}\right)$. For spectral classification, we first determined whether the stars were likely earlier or later than M3 using standard spectral indices. For later stars, we derived spectral types using spectral indices of TiO bands following Sicilia-Aguilar et al. (2008). Earlier stars were classified by comparing their spectra to Cep OB2 spectra described in Sicilia-Aguilar et al. (2005).

We estimate the spectral types of stars without spectra by deriving the best-fit effective temperature from model the optical to near-IR SED using the Robitaille et al. (2006) radiative transfer grid and then using the Currie et al. (2010) effective temperature scale for stars M2 or earlier and Luhman et al. (2003) for later stars. For some sources, we cannot derive a spectral type because the source lacks optical data or is a protostar. Tables 3 and 4 list the spectral types for each star.

To probe the mid-IR fluxes of bright stars that saturate the IRAC and MIPS detectors, we combined our source list with IRAS data for R CrA, TY CrA, and S CrA from Wilking et al. $(1985,1992)$. Our IRAC and MIPS mosaics indicate that the emission from $\mathrm{R} \mathrm{CrA}$ and S CrA clearly dominates over the background nebular emission and thus are only weakly contaminated. As mentioned previously, TY CrA is heavily contaminated by background emission. Given the large beam size of IRAS compared to Spitzer, the IRAS flux quoted for TY $\mathrm{CrA}$ is also likely unreliable. 

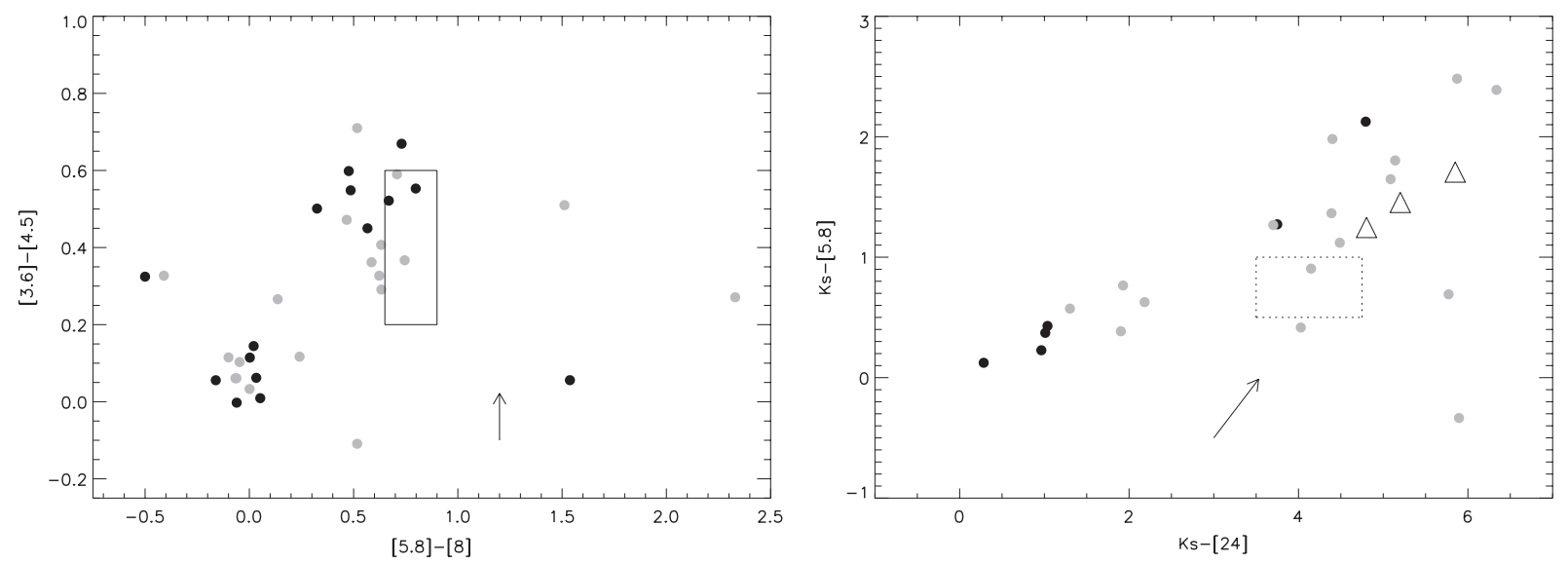

Figure 2. Left panel: IRAC color-color diagram of Coronet Cluster stars with new photometry (black circles) and those analyzed in Sicilia-Aguilar et al. (2008, gray circles). Right panel: $K_{s}-[5.8]$ vs. $K_{s}-[24]$ color-color diagram for Coronet Cluster stars. In each panel, we overplot a reddening vector of $A_{V}=10$, assuming the extinction relations of Indebetouw et al. (2005) for 2MASS and IRAC and Flaherty et al. (2007) for MIPS. In the left panel, the box corresponds to the range of colors for most stars in Taurus (Hartmann et al. 2005); the dotted box in the right-hand panel corresponds to the range of colors for most homologously depleted transitional disks in NGC 2362 identified by Currie et al. (2009). The triangles in the right panel correspond to (from bottom to top) the lower-quartile Taurus SED, median Taurus SED, and upper-quartile Taurus SED for K5-M2 stars from Furlan et al. (2006).

Table 4

Provisional Evolutionary States for Coronet Cluster Disks Studied in Sicilia-Aguilar et al. (2008) Based on 1-70 $\mu \mathrm{m}$ Data

\begin{tabular}{|c|c|c|c|c|c|c|c|c|}
\hline ID & ST & ST Ref. & $A_{V}$ (best) & $\tau_{\text {mid-IR }}$ & Inner Hole? $\left(R_{\text {sub }}\right)$ & $\begin{array}{c}\text { Disk State } \\
\quad(\mathrm{Si08)}\end{array}$ & $\begin{array}{c}\text { Disk State } \\
\text { (Er09) }\end{array}$ & $\begin{array}{c}\text { Disk State } \\
\text { (This Work) }\end{array}$ \\
\hline $\mathrm{CrA}-205$ & M4-M6 & 8,9 & 0.25 & Thin & $\mathrm{y}, 2000$ & $\mathrm{TD}(\mathrm{IH})$ & PD & $\mathrm{TD}(\mathrm{IH})$ \\
\hline $\mathrm{CrA}-432$ & M5-M7 & 8,9 & 0.5 & Thick & $\mathrm{n}$ & PD & $\ldots$ & PD \\
\hline $\mathrm{CrA}-453$ & M4.5 & 10 & 1.5 & & $\ldots$ & Star & $\ldots$ & Star \\
\hline CrA-465 & M5-M7.5 & 8,10 & 0 & Thick & $\mathrm{n}$ & PD & $\ldots$ & PD \\
\hline CrA-466 & M2 & 10 & 6.6 & Thick & $\mathrm{n}$ & $\mathrm{TD}(\mathrm{IH})$ & PD & PD \\
\hline CrA-4107 & $\geqslant \mathrm{M} 4.5$ & 8,9 & 1 & Thick & $\mathrm{n}$ & PD & $\ldots$ & PD \\
\hline CrA-4109 & $>\mathrm{M} 5$ & 9 , est. & 1 & Thin & $\mathrm{y}, 300$ & $\mathrm{TD}(\mathrm{IH})$ & $\mathrm{TD}(\mathrm{IH})$ & $\mathrm{TD}(\mathrm{IH})$ \\
\hline CrA-4110 & M5 & 9 & 0.4 & Thick & $\mathrm{n}$ & PD & & $\mathrm{PD}$ \\
\hline CrA-4111 & M4.5 & 9 & 0 & Thin & $\mathrm{y}, 70$ & $\mathrm{TD}(\mathrm{IH})$ & $\mathrm{TD}(\mathrm{IH})$ & $\mathrm{TD}(\mathrm{IH})$ \\
\hline G-1 & M0 & 9 , est. & 3 & Thin & 2 & PD & $\ldots$ & $\mathrm{TD}(\mathrm{HD})$ \\
\hline G-14 & M4.5 & 10 & 1.9 & Thin & $\mathrm{n}$ & $\mathrm{TD}(\mathrm{IH})$ & PD & $\mathrm{TD}(\mathrm{HD})$ \\
\hline G-30 & M3.5 & 10 & 0.09 & Thin & $\mathrm{n}$ & $\mathrm{TD}(\mathrm{IH}) / \mathrm{DD}$ & $\ldots$ & $\mathrm{TD}(\mathrm{HD})$ \\
\hline G-32 & $>$ M5 & 10, est. & $8-15$ & Thick & $\mathrm{n}$ & PD & $\ldots$ & PD \\
\hline G-49 & M4 & 10 & 0.07 & $\ldots$ & $\ldots$ & Star & $\ldots$ & Star \\
\hline G-65 & M1-M2 & 10 & $14-15$ & Thick & $\mathrm{n}$ & $\mathrm{TD}(\mathrm{IH})$ & PD & PD \\
\hline G-85 & M0.5 & 9 , est. & 17 & Thick & $\mathrm{n}$ & PD & $\ldots$ & PD \\
\hline G-87 & M1.5 & 10 & 14 & Thin & 1 & $\mathrm{TD}(\mathrm{IH})$ & PD & $\mathrm{TD}(\mathrm{HD})$ \\
\hline G-94 & M3.5 & 10 & 0.6 & Thin & $\mathrm{n}$ & $\mathrm{TD}(\mathrm{IH}) / \mathrm{DD}$ & $\ldots$ & $\mathrm{TD}(\mathrm{HD}) / \mathrm{DD}$ \\
\hline G-95 & M1 & 10 & 5 & Thin & $\mathrm{y}, 20$ & $\mathrm{TD}(\mathrm{IH}) / \mathrm{DD}$ & $\ldots$ & $\mathrm{TD}(\mathrm{IH}) / \mathrm{DD}$ \\
\hline G-102 & M5 & 10 & 0.7 & Thin & $\mathrm{n}$ & $\mathrm{TD}(\mathrm{IH}) / \mathrm{DD}$ & $\ldots$ & $\mathrm{TD}(\mathrm{HD}) / \mathrm{DD}$ \\
\hline
\end{tabular}

Notes. References for spectral types are the following: (8) López-Martí et al. 2005, (9) A. Sicilia-Aguilar et al. 2011, in preparation, (10) Sicilia-Aguilar et al. 2008. "Est" means that the spectral type was estimated by modeling the optical to near-IR SED using the Robitaille et al. (2006) grid and the Currie et al. (2010) effective temperature scale.

\subsection{IRAC and MIPS Colors and Observed SEDs of Coronet Cluster Stars}

To provide a first-order investigation of the Coronet Cluster disk population, we analyze the distribution of near-to-midIR colors and optical/IR SEDs. The left panel of Figure 2 displays the observed IRAC colors of cluster stars with new photometry (black dots) and stars analyzed by Sicilia-Aguilar et al. (2008, gray dots). The distributions appear similar. Many stars concentrate near zero color (as expected for bare stellar photospheres) and around [3.6] $-[4.5]=0.3-0.6$ and $[5.8]-[8]=0.5-0.7$. Compared to the typical IRAC colors of primordial disks in 1-2 Myr old clusters (solid box; Hartmann et al. 2005), colors for Coronet Cluster stars may be slightly bluer by $\sim 0.2$ mag.
The longer wavelength colors for cluster stars exhibit a wider diversity (Figure 2, right panel). At least three sources, all from the Sicilia-Aguilar et al. (2008) sample, show evidence for weak/negligible $5.8 \mu \mathrm{m}$ excess but a $\gtrsim 4$ mag excess at $24 \mu \mathrm{m}$ characteristic of transitional disks with inner holes. Others have $24 \mu \mathrm{m}$ and/or $5.8 \mu \mathrm{m}$ excess emission characteristic of more homologously depleted transitional disks, such as those found in NGC 2362 (dotted enclosed region; Currie et al. 2009), which is weaker than emission for the lower-quartile median Taurus SED from Furlan et al. (2006, lower-left triangle). The cluster also includes many stars with strong IRAC and MIPS excesses comparable to the median Taurus and upper-quartile Taurus SED. Because only six stars in the new sample have $K_{s}$, [5.8] and [24] detections, it is less clear from the $K_{s}-[5.8] / K_{s}-[24]$ 




Figure 3. Spectral energy distributions of Coronet Cluster stars with new Spitzer photometry. In most cases, the SEDs can be modeled as a stellar photosphere (green) plus excess emission from a disk. The stellar photospheres plotted come from the MARCS stellar atmosphere models (Gustafsson et al. 2008, and references therein). Sources not shown lack near-to-mid-IR data required to constrain their SEDs.

(A color version of this figure is available in the online journal.)

diagram whether the distribution of colors from the new cluster sample displays the same diversity.

Examining the SEDs of new sources clearly shows that they have a wide range of morphologies (Figure 3). Many sources (e.g., IRS2, IRS5ab, and IRS7w) have rising near-to-mid-IR SEDs indicative of Class I protostars. Well-known sources with optically thick disks, such as the intermediate-mass stars S CrA and R CrA, have nearly flat SEDs from $1 \mu \mathrm{m}$ to $8 \mu \mathrm{m}$.

IRS spectra strengthen our identification of sources whose photometric data alone make them difficult to classify. In particular, V709 shows evidence for a weak excess based on its MIPS $24 \mu \mathrm{m}$ flux and IRS spectra. Its SED gradually peels away from the stellar photosphere from $\sim 8 \mu \mathrm{m}$ to $30-35 \mu \mathrm{m}$. Based on their $10 \mu \mathrm{m}$ absorption features, IRS5 and IRS7 $\mathrm{w}$ are likely protostars with cool envelopes (e.g., Willner et al. 1982; Andre \& Montmerle 1994), consistent with previous classifications (Henning et al. 1994; Chini et al. 2003; Groppi et al. 2007).

\section{ANALYSIS}

To assess the evolutionary states of Coronet Cluster disks, we analyze source SEDs from the new sample and the SiciliaAguilar et al. (2008) sample using both simple theoretical comparisons and sophisticated radiative transfer modeling. We follow a slightly modified version of the analysis methods used in Currie et al. (2009) to distinguish disks in different states. These classifications are based solely on 1-24 $\mu \mathrm{m}$ data: since far-IR/submillimeter data provide crucial constraints on disk properties, in particular the inferred disk mass, we consider these classifications to be provisional. We will revisit these classifications later after analyzing stars in other clusters with longer wavelength data (Section 4).

In our classification, we consider protostars and disks with three main evolutionary states: primordial disks, transitional disks, and debris disk candidates. We identify protostars as sources with rising SEDs from the near-IR to mid-IR consistent with emission predominantly from a cold dusty envelope $(T \leqslant$ 200-300 K). Primordial disks are disks that are optically thick $\left(\tau_{\mathrm{IR}} \gg 1\right)$ and thus lack evidence for dust evolution consistent with active disk clearing.

Transitional disks are disks that show substantial dust evolution consistent with active disk clearing. They can have one of two morphologies. First, they can exhibit a drop in the optical depth ( $\tau \approx 1$ or less) of emitting dust either at all IR wavelengths consistent with a reduced disk mass (a homologously depleted transitional disk). Second, they can also have photospheric or weak, optically thin emission at shorter wavelengths (e.g., 3.6-5.8 $\mu \mathrm{m}$ ) and more optically thick emission at longer 

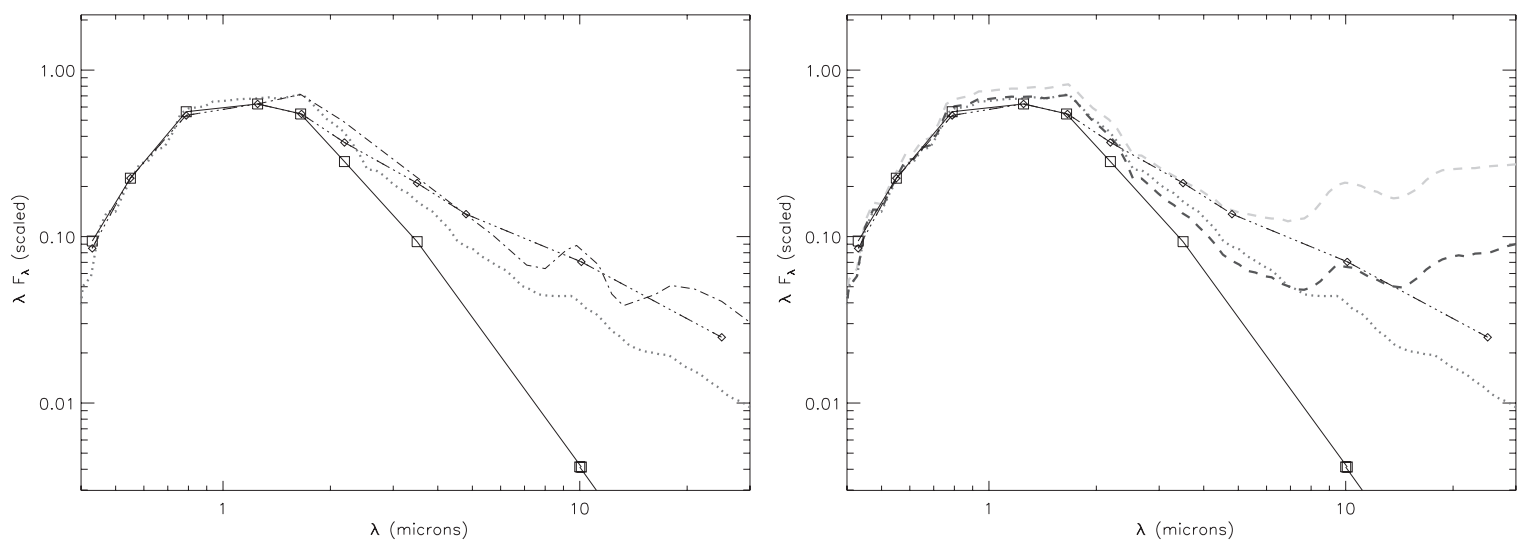

Figure 4. Comparisons between various empirical and theoretical SEDs and our fiducial flat disk models used for analysis-the Kenyon \& Hartmann (1987) flat, reprocessing disk and our non-flaring, optically thick reprocessing disk from the Whitney et al. (2003a) Monte Carlo radiative transfer code. The models shown are for M0, $T_{e}=3850 \mathrm{~K}$ stars. In both panels, the Kenyon \& Hartmann (1987) disk is shown as a dashed-triple-dots-diamonds locus, the Whitney et al. model is shown as a gray dotted line, and the stellar photosphere is shown as a solid line connected by squares. Left: comparisons with the lower-quartile Taurus SED from Furlan et al. (dash/dots 2006). Right: comparisons with a standard flared disk model (gray dashed line) and a flared but "settled"/shadowed disk model (black dashed line). The Whitney radiative transfer models deviate from the stellar photosphere and Kenyon \& Hartmann flat disk in the near-IR because it includes strong, hot, optically thick emission from the puffed up inner disk regions.

Table 5

IRS Flux Density Measurements Used in Modeling Coronet Cluster Stars from Sicilia-Aguilar et al. (2008)

\begin{tabular}{lcc}
\hline \hline \multicolumn{1}{c}{ ID } & Wavelengths Sampled $(\mu \mathrm{m})$ & Flux Density $(\mathrm{mJy})$ \\
\hline CrA-205 & $9,13,17,21,27$ & $1.03 \pm 0.06,0.70 \pm 0.08,0.91 \pm 0.30,1.10 \pm 0.30,2.08 \pm 0.26$ \\
G-14 & " & $4.95 \pm 0.20,4.70 \pm 0.15,5.53 \pm 0.45,4.75 \pm 0.34,3.62 \pm 0.49$ \\
G-65 & $6,7,9,10,11,12$ & $53.06 \pm 12.80,48.30 \pm 30.30,49.96 \pm 13.27,47.9 \pm 12.30,48.10 \pm 23.98,43.1 \pm 32.70$ \\
G-87 & $9,13,17,21,27$ & $10.02 \pm 0.31,12.56 \pm 0.29,15.55 \pm 4.84,19.04 \pm 4.88,25.37 \pm 6.19$ \\
\hline
\end{tabular}

Note. The wavelength sampling for G-65 is different than the others because G-65 lacks data longward of $\sim 15 \mu \mathrm{m}$.

wavelengths consistent with inside-out disk clearing (a transitional disk with an inner hole). In this latter group, we include the so-called pre-transitional disks (Espaillat et al. 2007). These disks have a small amount of high optical depth material very close to the star but otherwise have large, dust-poor inner regions (e.g., large gaps) and optically thick outer regions and thus also identify an inside-out disk clearing.

Debris disk candidates lack evidence for accretion and have very optically thin emission $\left(\tau_{\mathrm{IR}} \ll 1\right)$ from a low mass $\left(\lesssim 0.01 M_{\text {lunar }}\right.$; Chen et al. 2005) of second-generation dust (Backman \& Paresce 1993; Currie et al. 2008; Kenyon \& Bromley 2008). Many of the Coronet Cluster stars are $\mathbf{M}$ dwarfs with very low masses. Even if these stars have tenuous emission and lack evidence for accretion, they also have grain removal timescales comparable to the host star's age. Thus, their dust need not be second generation (Currie \& Kenyon 2009), they could be very late stage transitional disks, and at best can only be considered debris disk candidates. Our basis for identifying sources with tenuous $24 \mu \mathrm{m}$ excess emission as debris disk candidates follows Carpenter et al. (2009) and is purely empirical. They identify debris disk candidates around late-type stars as those with $0.25-0.524 \mu \mathrm{m}$ excesses. In our analysis, we consider the evolutionary state of these tenuous disks around Coronet Cluster stars as indeterminable and simply label them as transitional disks/debris disk candidates. ${ }^{3}$

\footnotetext{
3 While some debris disks have much stronger emission, nearly all of them surround what are (or will be) main-sequence A stars or early F stars (e.g., Rieke et al. 2005; Currie et al. 2008) that have masses $\gtrsim 1.5 M_{\odot}$. Debris disks detected around lower-mass stars (the focus of this paper) are far more infrequent and typically have $24 \mu \mathrm{m}$ excesses of only a few tenths of magnitudes (e.g., Plavchan et al. 2009). Our results are insensitive as to whether or not stars with tenuous excesses are considered to have debris disks or transitional disks.
}

For most sources, only photometric data are available. For sources with IRS data from Sicilia-Aguilar et al. (2008), we add the IRS flux densities, which when combined with our photometry yields flux densities at equally spaced wavelengths (Table 5). The inclusion of the IRS data provides better sampling of the SED from 5 to $24 \mu \mathrm{m}$ and extends the wavelength coverage.

\subsection{Method for Disk Identification}

\subsubsection{Fiducial SEDs}

We first compare source SEDs to geometrically flat, optically thick models to identify disks with evidence for a reduced optical depth of emitting material $\left(\tau_{\mathrm{IR}} \lesssim 1\right)$. Figure 4 shows two flattened reprocessing disk models appropriate for a $T_{e}=$ $3850 \mathrm{~K}$ primary (M0 spectral type), though SEDs for earlier and later stars (e.g., K5-M6) yield similar mid-IR fluxes relative to the stellar photosphere. The first model (dash-tripledots/diamonds) is the standard, razor-thin flat reprocessing disk model from Kenyon \& Hartmann (1987, see also Adams et al. 1987), truncating the inner disk at a dust sublimation temperature of $T=1500 \mathrm{~K}$. The second model (thick gray dashed line) is produced from the Whitney-Monte Carlo radiative transfer code (Whitney et al. 2003a, 2003b; Robitaille et al. 2006). The model assumes a disk mass of $0.05 M_{\odot}$, no flaring $(H / r=$ constant), no accretion, and no protostellar envelope emission. The disk is optically thick to its own radiation over spatial scales relevant for our study ( $\lesssim 10 \mathrm{AU})$.

The Whitney disk model consistently has weaker disk emission than the simple Kenyon \& Hartmann flat disk model, because stellar photons in the Whitney model are partially attenuated before they reach the $\tau=1$ surface, due to the disk's 

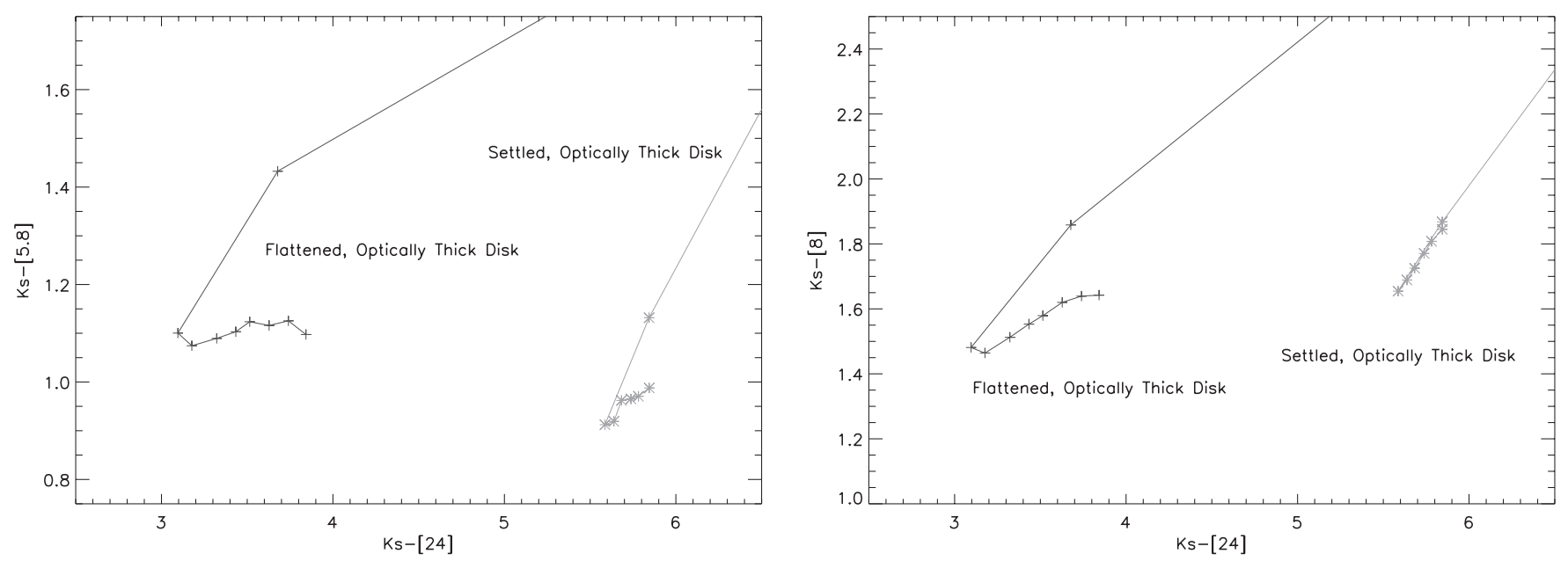

Figure 5. Loci of colors for an optically thick flattened disk around an M0 star (black solid line) and an optically thick "settled" disk around an M0 star (gray solid line) as a function of disk inclination. Disks viewed edge-on have extremely red colors and lie off the boundary of the plot. The rightmost point in each plot corresponds to the face-on case. Note that since the model predicts some $K$-band excess, the colors compared with that of the stellar photosphere are redder by an additional $\sim 0.1-0.2 \mathrm{mag}$.

finite thickness. Thus, the disk is colder at a given location and reradiates less energy. To provide an empirical comparison for source SEDs, we overplot the lower-quartile Taurus SED (Figure 4, left panel) from Furlan et al. (2006), which has been used in other work to help distinguish disks in different states (Cieza et al. 2008; Currie et al. 2009; Currie \& Kenyon 2009). The lower-quartile Taurus SED almost perfectly tracks the Kenyon \& Hartmann flat disk model.

In the right panel of Figure 4, we compare our fiducial SEDs to a flared optically thick disk model (thick gray long-dashed line) with $H / r=r^{2 / 7}$ (e.g., Chiang \& Goldreich 1997, 1999) using the Whitney code and a disk that has undergone significant dust settling (thick black long-dashed line). Following Lada et al. (2006), we quantify dust settling by reducing the disk scale height by a factor of three compared to the thermal equilibrium value adopted in the flared disk model. ${ }^{4}$ The Whitney settled disk model predicts emission roughly equal to the Whitney flat disk model through $\sim 7 \mu \mathrm{m}$ and greater emission at longer wavelengths in spite of being flared: this probably happens because the puffed up inner wall effectively attenuates flux from disk regions slightly exterior (e.g., the disk only comes out of shadow at regions that effectively emit at $\lambda>7 \mu \mathrm{m})$.

In Figure 5, we plot the $K_{s}-[5.8,8]$ versus $K_{s}-[24]$ colors for the flattened and settled disk models as a function of disk inclination, produced by convolving the model output with 2MASS and Spitzer filter functions provided as a part of the Whitney code. There is little variation in color, especially for the flat model, except when the disk is viewed nearly edge-on. In this case, the disk heavily extincts the star, causing its observed colors to be far redder than in face-on cases. The loci of colors indicate that flattened optically thick disks should typically have $K_{s}-[5.8,8,24]>1.1,1.5,3.5$. Settled disks have slightly bluer $K_{s}-[5.8]$ colors $(\sim 0.85-1)$ but redder $K_{s}-[8,24]$ colors (1.6-1.9). For the models where the disk is not viewed edge-

\footnotetext{
4 Technically, we assume less "settling" than in other "settled disk" models, like those presented in D'Alessio et al. (2006). However, their treatment of settling assumes that some small, strongly emitting grains remain at larger heights above the disk midplane, whereas the Whitney code lacks this superheated grain population and thus may underpredict near-to-mid-IR disk emission. Furthermore, some of the D'Alessio et al. (2006) "settling" models likely produce weak emission because they assume low disk masses, in addition to being "settled."
}

on, the disks have $K$-band excess emission of $\sim 0.1-0.2 \mathrm{mag}$, consistent with a visual inspection of Figure 4. Thus, optically thick, flattened disks lacking $K$-band excess (e.g., because of submicron-sized grain growth), should then typically have $K_{s}-$ [5.8] $\gtrsim 1.2$ and $K_{s}-[8] \gtrsim 1.6-1.7$. The colors for an optically thick flared disk lie off the plot range (e.g., $K_{s}-[8]=$ $\left.\left.2.75, K_{s}-24\right]=6.75\right)$. The disk emission at $24 \mu \mathrm{m}$ varies wildly with disk flaring, so MIPS $24 \mu \mathrm{m}$ does not effectively probe the disk optical depth. The colors from disk models for K5-M6 stars agree to within $\sim 0.05-0.1 \mathrm{mag}$.

Since the Whitney flat disk model produces weaker emission through $8 \mu \mathrm{m}$ than the other fiducial models, it defines a conservative limit for disks with optically thick emission. Because we identify primordial disks as those with optically thick IR emission, disks with 2-8 $\mu \mathrm{m}$ emission greater than this flat disk model are consistent with being primordial disks. Conversely, disks with weaker emission correspond to later evolutionary stages: transitional disks and debris disk candidates.

We emphasize that our adopted optically thick disk limit is conservative. Emission in excess of this limit at $\lambda \sim 5-8 \mu \mathrm{m}$ may be explained by disk flaring. While the model used to produce this limit lacks flaring, real disks almost assuredly have some flaring. Furthermore, the IRAC $8 \mu \mathrm{m}$ channel overlaps with the $10 \mu \mathrm{m}$ silicate feature, complicating comparisons with the flat disk limit (see Muzerolle et al. 2010). The model also lacks accretion luminosity, which contributes some IR flux, but many disks we model show unambiguous accretion signatures.

\subsubsection{SED Modeling}

After comparing source SEDs to our flat reprocessing disk SED, we model optical-to-mid-IR fluxes with the grid of radiative transfer disk models from Robitaille et al. (2006), which yields estimates for disk properties (e.g., mass and inner radius) consistent with other independent work (see Robitaille et al. 2007). By identifying the best-fitting models $\left(\min \left(\chi^{2}\right)\right)$, we determine whether the disk plausibly has an inner region cleared of dust and is thus consistent with being a transitional disk with an inner hole. The computed inner disk radius distinguishes disks that lack inner holes (primordial, homologously depleted transitional disk) and transitional disk with inner holes. To be conservative, we set the division at 10 times the dust 


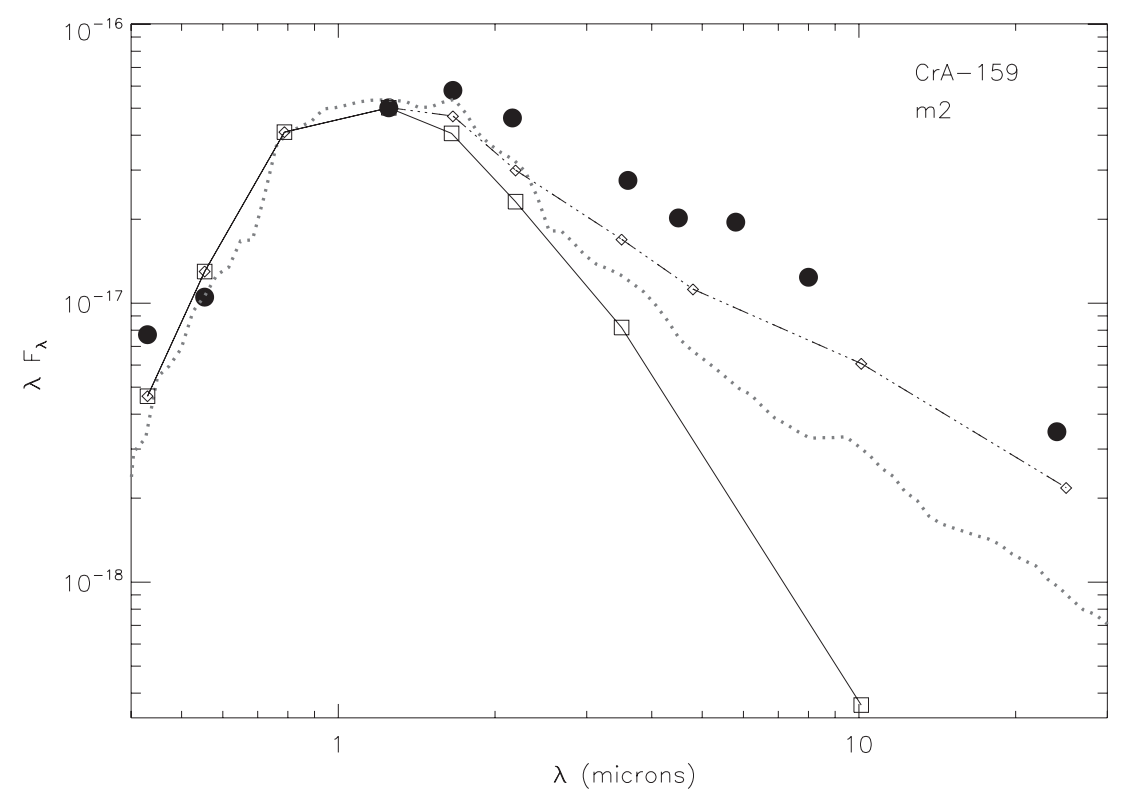

Figure 6. SED analysis for CrA-159, comparing its emission to the razor-thin flat optically thick disk model (e.g., Kenyon \& Hartmann 1987, triple-dash dots/ diamonds) and a flat, optically thick disk model constructed from the Whitney radiative transfer code (dotted line). For reference, we overplot the stellar photosphere as a solid line. CrA-159 has disk emission consistently lying above the Whitney flat disk model and thus we classify it as having a primordial disk.

sublimation radius: disks with $R_{\text {in }} \geqslant 10 R_{\text {sub }}$ are identified as transitional disks with inner holes. To account for flux calibration uncertainties, photometric errors, and variability, we assume a minimum $10 \%$ flux uncertainty in each photometric filter and IRS monochromatic flux density.

We restrict ourselves to disk models which provide a good fit, which we define as $\chi^{2}-\chi^{2}$ best $<3$, where $\chi^{2}$ best corresponds to the minimum $\chi^{2}$ per data point. This criterion is very similar to that used in previous studies (e.g., Robitaille et al. 2007; Currie et al. 2009; Ercolano et al. 2009). Finally, we incorporate systematic uncertainties in the extinction $A_{V}$ and distance. For the extinction, we nominally assume a $20 \%$ systematic uncertainty with a "floor" of $\pm 0.5 \mathrm{mag}$. For the distance, we assume $150 \mathrm{pc}$ with an uncertainty of $\pm 20 \mathrm{pc}$.

\subsection{Results}

Tables 3 and 4 describe our provisional disk modeling results. The combined sample from both tables consists of five protostars, 16 stars with primordial disks, six with homologously depleted transitional disks, four that have transitional disks with inner holes, and six bare stellar photospheres. Sources not analyzed here lack sufficient photometric data to model their SEDs and/or determine their plausible range of spectral types (e.g., FP-8, FP-33, FP-37, and FP-38), making it impossible to identify their disk evolutionary states. Below we discuss modeling of several sources illustrating how we determine disk states.

\subsubsection{Disks in Different Evolutionary States}

Figure 6 compares the SED for CrA-159 from the new sample to the Kenyon \& Hartmann razor-thin flat disk model and the flat disk model produced from the Whitney radiative transfer code. The observed SED is dereddened from $A_{V}=3$. CrA159 has an infrared excess detectable from $1.6-2 \mu \mathrm{m}$ to $24 \mu \mathrm{m}$, which consistently lies well above the Whitney flat disk model, indicating that its emission is optically thick. According to our criteria, this source then has a primordial disk. With the exception of one star (V709), all disks in the new Coronet sample, predominately comprised of higher-mass stars, yield emission lying above the flat, optically thick disk limit.

The disk population for the Sicilia-Aguilar et al. (2008) sample is far more diverse, including many transitional disks. We show comparisons between three homologously depleted transitional disk SEDs and flat disk models in Figure 7. Each source has a reduced level of disk emission compared flat disk models. Likewise, all transitional disks with inner holes have weak/negligible 3.6-5.8 $\mu \mathrm{m}$ emission but optically thick $24 \mu \mathrm{m}$ emission.

The top panel of Figure 8 shows model fits for CrA-205, which has a transitional disk with an inner hole. CrA-205 exhibits no excess emission shortward of $\sim 10 \mu \mathrm{m}$ but has a rising SED clearly departing from the stellar photosphere by $\sim 15 \mu \mathrm{m}$. All of the best-fitting Robitaille models have inner disk radii $\left(R_{\text {in }}>1000 R_{\text {sub }}\right)$ much larger than our threshold identifying transitional disks with inner holes $\left(10 R_{\mathrm{sub}}\right)$. Moreover, the grid sampling of inner disk radii (gray shaded region) is heavily peaked at $1 R_{\text {sub }}$ (e.g., no inner hole). In spite of this intrinsic bias against selecting disk models with holes, all disk models lacking inner holes fail our $\chi^{2}$ threshold.

Our results for the Sicilia-Aguilar et al. sample are intermediate between those of Sicilia-Aguilar et al. (2008) and Ercolano et al. (2009) who disagreed over the fraction of transitional disks with inner holes. CrA-466 and G-65 have best-fit models that do not require an inner hole (Figure 8 , middle and bottom panels). Like Ercolano et al. (2009), we identify them as primordial disks. However, our modeling supports the claim by SiciliaAguilar et al. $(2008,2009)$ that CrA-205 has an inner hole, in fact the largest one in our sample. Ercolano et al. (2009) did not to identify CrA-205's inner hole probably because they do not include the IRAC $8 \mu \mathrm{m}$ data nor any IRS data in their fitting.

While we also agree with Ercolano et al. (2009) that G-14 and G-87 lack evidence for an inner hole, our modeling suggests that both have weaker emission than a perfectly flat, optically thick reprocessing disk. Thus, like Sicilia-Aguilar et al. (2008) we identify these as transitional disks, albeit ones that are homologously depleted. The Ercolano et al. (2009) 

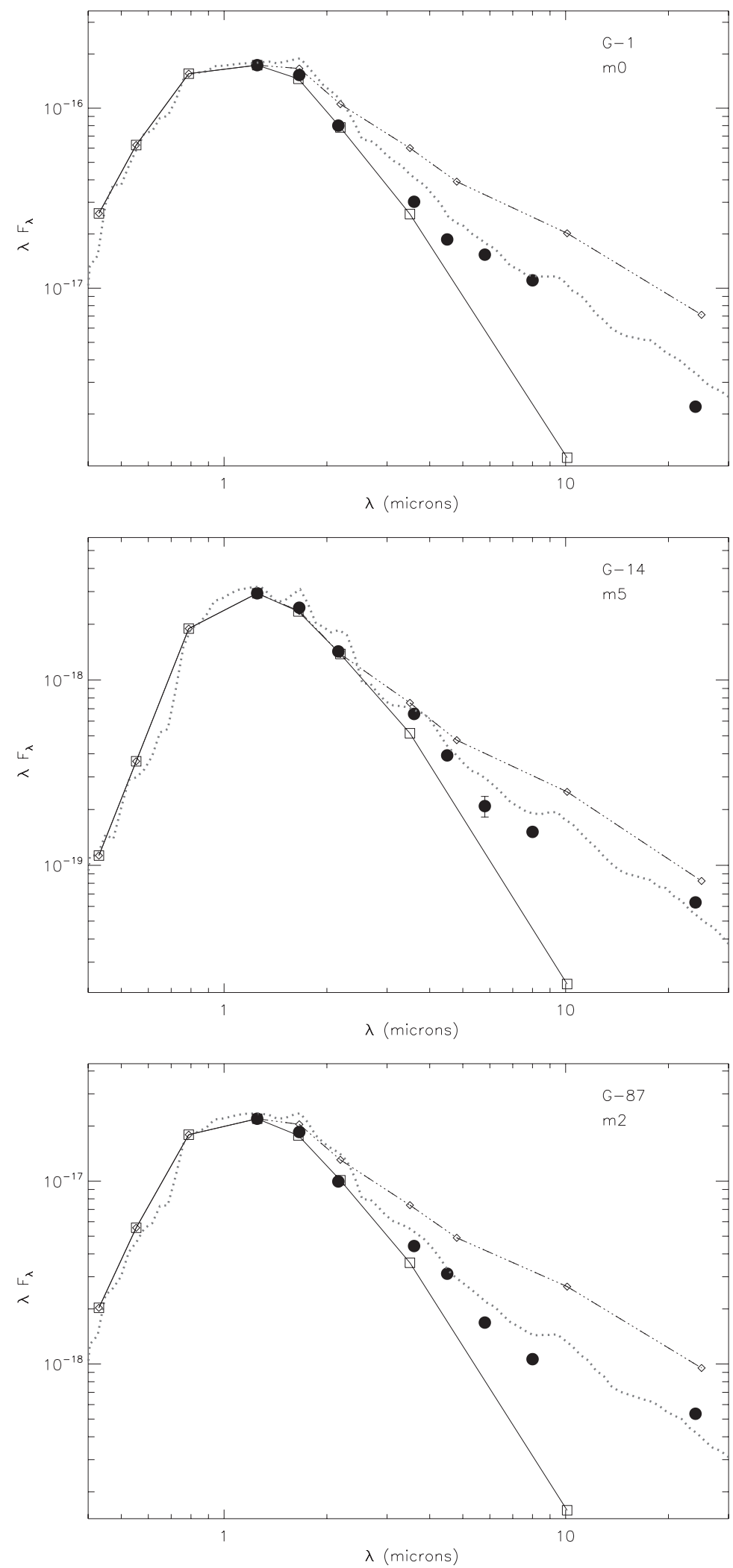

Figure 7. SEDs G-1 (top), G-14 (middle), and G-87 (bottom). We fit the optical/near-IR portion of the SED to a stellar SED constructed from the T. Currie et al. (2011, in preparation) intrinsic colors (solid line), overplot the flat, optically thick disk model constructed from the Whitney radiative transfer code (dotted line) and overplot the flat, reprocessing disk SED from Kenyon \& Hartmann (1987, triple-dash dots). All three stars have homologously depleted transitional disks.

study focused on identifying transitional disks with inner holes. Therefore, we identify more transitional disks because we adopt a more expansive definition for what constitutes a transitional disk.
Two of the sources labeled as homologously depleted transitional disks may be debris disk candidates, while one of the transitional disks with inner holes may be a debris disk candidate. The identity of V709 is particularly questionable: while 

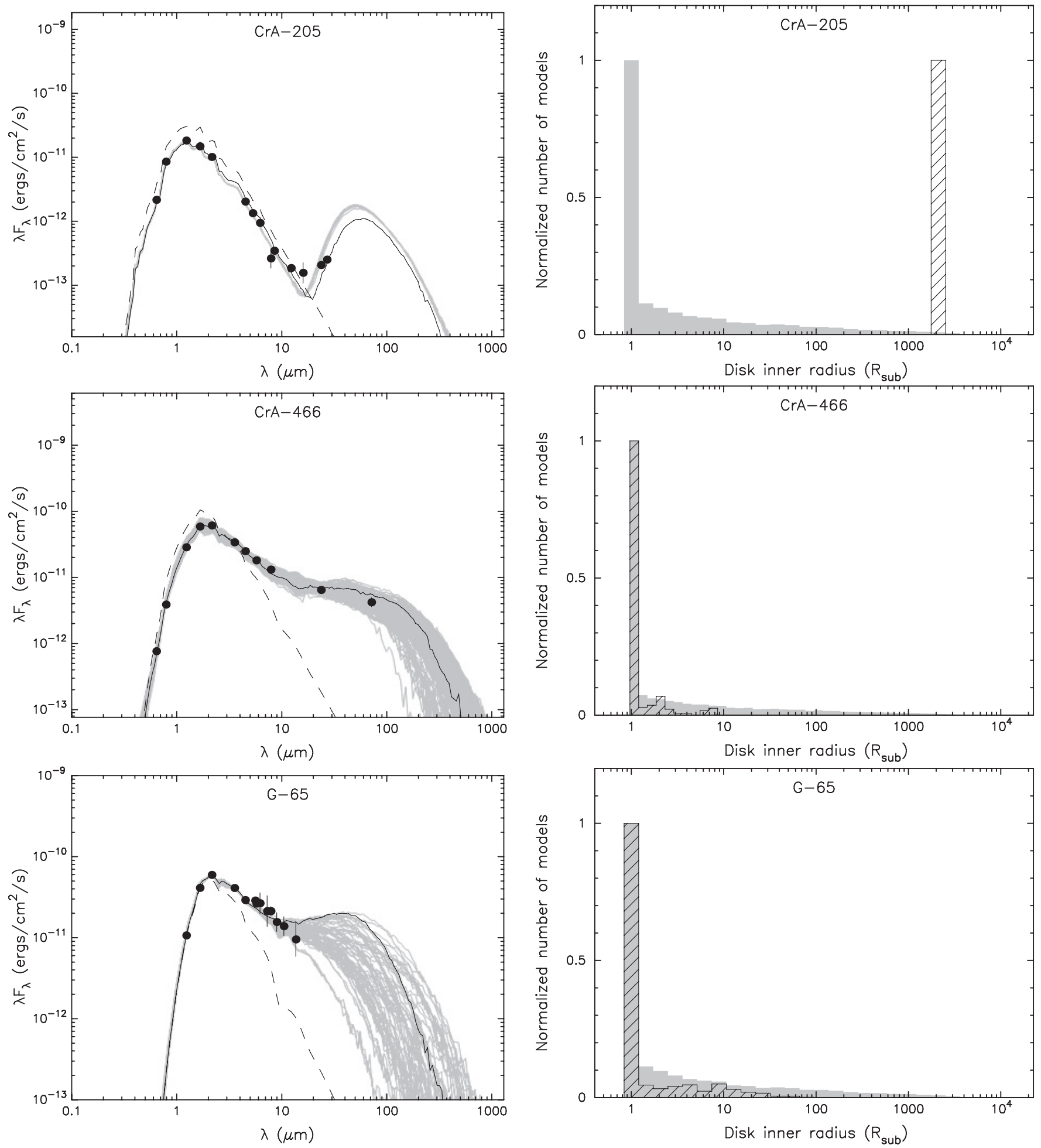

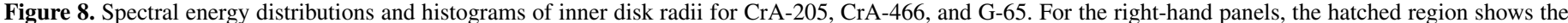

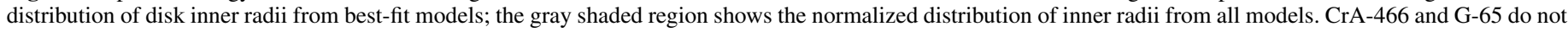

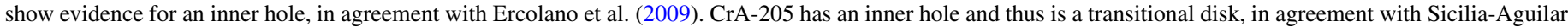
et al. (2008).

we identify it as a disk-bearing star, it is possible that its weak excess could be due to nebular contamination or an offset between the near-IR data and the MIPS data/IRS spectra caused by variability such as that produced by an undetected eclipsing companion. More importantly, if it has a disk, it is unclear whether it is a debris disk or transitional disk.
Cluster members with new Spitzer photometry are mostly protostars or stars with primordial disks (5 and 8, respectively, Table 3). The disk population for $\mathrm{M}$ stars sample studied in Sicilia-Aguilar et al. (2008) is more evenly divided between primordial disks (8) and transitional disks (3-4 with inner holes and 4-6 homologously depleted). Unlike the new sample, many 
stars in the Sicilia-Aguilar et al. (2008) sample have homologously depleted transitional disks. Thus, based on modeling $1-24 / 70 \mu \mathrm{m}$ data, the percentage of protoplanetary (primordial + transitional) disks in our combined sample that are transitional disks is $\sim 30 \%$ (7/23). The percentage rises to $41 \%$ if debris disk candidates are classified as transitional disks, including V709 $(11 / 27)$.

\subsubsection{Disk Properties as a Function of Stellar Mass}

To examine the spectral-type/stellar-mass dependence of disk properties, we bin our sample into three spectral-type groups: earlier than K5, K5-M2, and later than M2. Assuming the Baraffe (1998) isochrones (mixing length $=1.9 H_{p}$ ) and the $T_{e}$ versus spectral-type scale from Currie et al. (2010), the corresponding stellar-mass division is $M_{\star}>1 M_{\odot}, M_{\star}=$ $0.5-1 M_{\odot}$, and $M_{\star}<0.5 M_{\odot} .^{5}$

The transitional disk frequency for Coronet Cluster members may be spectral-type dependent. At most one and probably none of the disks around stars earlier than K5 appear to be transitional disks (0-1/5). Stars between K5 and M2 also mostly have primordial disks (7/10) with 2-3 stars harboring transitional disks and 0-1 with debris disks. Accounting for the uncertain status of debris disk candidates, the relative fraction of transitional disks to all protoplanetary disks for stars M2 or earlier with plausible masses equal to $0.5 M_{\odot}$ or greater is then $f(\mathrm{TD}) /(f(\mathrm{TD})+f(\mathrm{PD})) \approx 0.15-0.27(2 / 13,4 / 15)$. For K5-M2 stars only, $22 \%-30 \%$ of the protoplanetary disks are transitional disks. Transitional disks are more prevalent around stars later than M2, comprising 50\%-58\% of the protoplanetary disk population $(5 / 10$ or $7 / 12)$. This high frequency is consistent with previous results from Sicilia-Aguilar et al. (2008).

However, given the small population of Coronet Cluster stars studied here it is unclear whether our analysis truly identifies a statistically significant stellar-mass-dependent frequency of transitional disks characteristic of most young clusters. Furthermore, it is possible that some transitional disks provisionally identified from modeling optical-to-mid-IR data may instead be primordial disks whose weak emission is due to the growth and settling of submicron-sized grains (e.g., Furlan et al. 2006). Analyzing data from clusters where far-IR and submillimeter data is available is required to more conclusively break these degeneracies. To explore these issues further, and provide a context for our results, we now analyze disks in other clusters.

\section{TRANSITIONAL DISK FREQUENCIES AND LIFETIMES AS A FUNCTION OF STELLAR MASS: A COMPARISON WITH TAURUS, IC 348, NGC 2362, AND $\eta$ CHA}

\subsection{Sample and Wavelength Ranges Used For Analysis}

To investigate the evolution of the transitional disk frequency with time, we analyze data in other clusters spanning an age range of 1-8 Myr. Comparing our results for the Coronet Cluster with 1-2 Myr old Taurus and 2-3 Myr old IC 348 addresses whether a stellar-mass-dependent transitional disk frequency is a general feature of 1-3 Myr old clusters. By comparing

\footnotetext{
5 In making these divisions, we are grouping together intermediate-mass $\left(M_{\star} \sim 2-4 M_{\odot}\right)$ and solar-mass T Tauri stars. Grouping together these stars as the first spectral-type bin may hide additional evolutionary trends since disk evolution for intermediate-mass stars $\left(\gtrsim 2-3 M_{\odot}\right)$ and solar-mass stars may proceed at different rates even by 1-3 Myr (e.g., Currie \& Kenyon 2009). Any differences between $1-4 M_{\odot}$ stars and lower-mass stars then applies only to the ensemble average of $1-4 M_{\odot}$ stars.
}

these results with those for 5 Myr old NGC 2362 and 6-8 Myr old $\eta$ Cha, we investigate how the frequency of transitional disks evolves with time and estimate the typical transitional disk lifetime.

Taurus. Members of the Taurus-Auriga star-forming region are listed in catalogs from Kenyon et al. (2008), Rebull et al. (2010), and Luhman et al. (2009), which are updates of the classic Kenyon \& Hartmann (1995) catalog. We reanalyze Taurus members studied in Luhman et al. (2010) to provide direct comparisons with their disk analysis. For Spitzer photometry, we use the IRAC and MIPS $24 \mu \mathrm{m}$ data from Luhman et al. (2010) and MIPS $70 \mu \mathrm{m}$ and $160 \mu \mathrm{m}$ data from Rebull et al. (2010). We adopt optical photometry from Kenyon \& Hartmann (1995), White \& Ghez (2001), and Audard et al. (2007) and submillimeter measurements from Andrews \& Williams (2005), Jewitt (1994), Jensen et al. (1994), and Beckwith \& Sargent (1991). To deredden stars, we determine optical extinctions for Taurus members, using estimates from Furlan et al. (2006), Rebull et al. (2010), Kenyon \& Hartmann (1995), and Luhman et al. (2010) as starting points and then fit the optical/near-IR portion of the SED to a synthetic SED produced using the Currie et al. (2010) intrinsic colors and the Currie et al. (2010) and Luhman et al. (2003) effective temperature scales.

A large number of Taurus sources have complete SEDs from optical to far-IR/submillimeter wavelengths. We model the SEDs of 25 K5-M6 stars whose IR colors plausibly identify both primordial disks and transitional disks based on various color-color selection criteria in the literature (e.g., Lada et al. 2006; Currie \& Kenyon 2009; Luhman et al. 2010; Muzerolle et al. 2010). ${ }^{6}$ Quantitatively, our selection criterion includes K5-M6 sources with $K_{s}-$ [8] (dereddened) $=1.25-2$. We further restrict our sample to sources whose far-IR/submillimeter data can yield good constraints on the range of plausible disk masses (see the Appendix). Specifically, we require that the source has published submillimeter data or a detection in the farIR MIPS bandpasses $(70 \mu \mathrm{m}$ or $160 \mu \mathrm{m})$. While this criterion may bias our selection against including the most depleted disks, especially for the lowest mass stars, we include it to be conservative.

IC 348. For IC 348, we compile optical, near-IR, and Spitzer/ IRAC photometry, spectral types and extinctions presented in Lada et al. (2006, and references therein) and MIPS photometry and upper limits presented in Currie \& Kenyon (2009) for 307 members listed in both studies as well as data for 41 additional spectroscopically confirmed members from Muench et al. (2007). We restrict our analysis to sources with MIPS $24 \mu \mathrm{m}$ detections. Currie \& Kenyon (2009) identified mid-IR colors for disks in different evolutionary states by modeling the SEDs of newly detected disks in IC 348, but their sample is comprised mainly of stars later than M3-M4. Here, we model the SEDs of 25 IC 348 stars previously detected by Lada et al. (2006) and Muench et al. (2007) with full SEDs through $24 \mu \mathrm{m}$ focusing on those with the same range of mid-IR colors analyzed in Taurus. Most of these 25 stars are earlier than M3-M4.

As with the Taurus sample, we use the published extinction estimates as a starting point and fit the optical/near-IR portion of the SED to arrive at a final value. In most cases, the best-fit optical extinction $\left(A_{V}\right)$ matches that listed by Lada et al. (2006). Exceptions include IDs 26, 58, 97, 110, and 9024: their new extinction estimates are generally larger.

\footnotetext{
6 We do not present modeling results for sources such as DM Tau, whose classification as a transitional disk has broad agreement.
} 

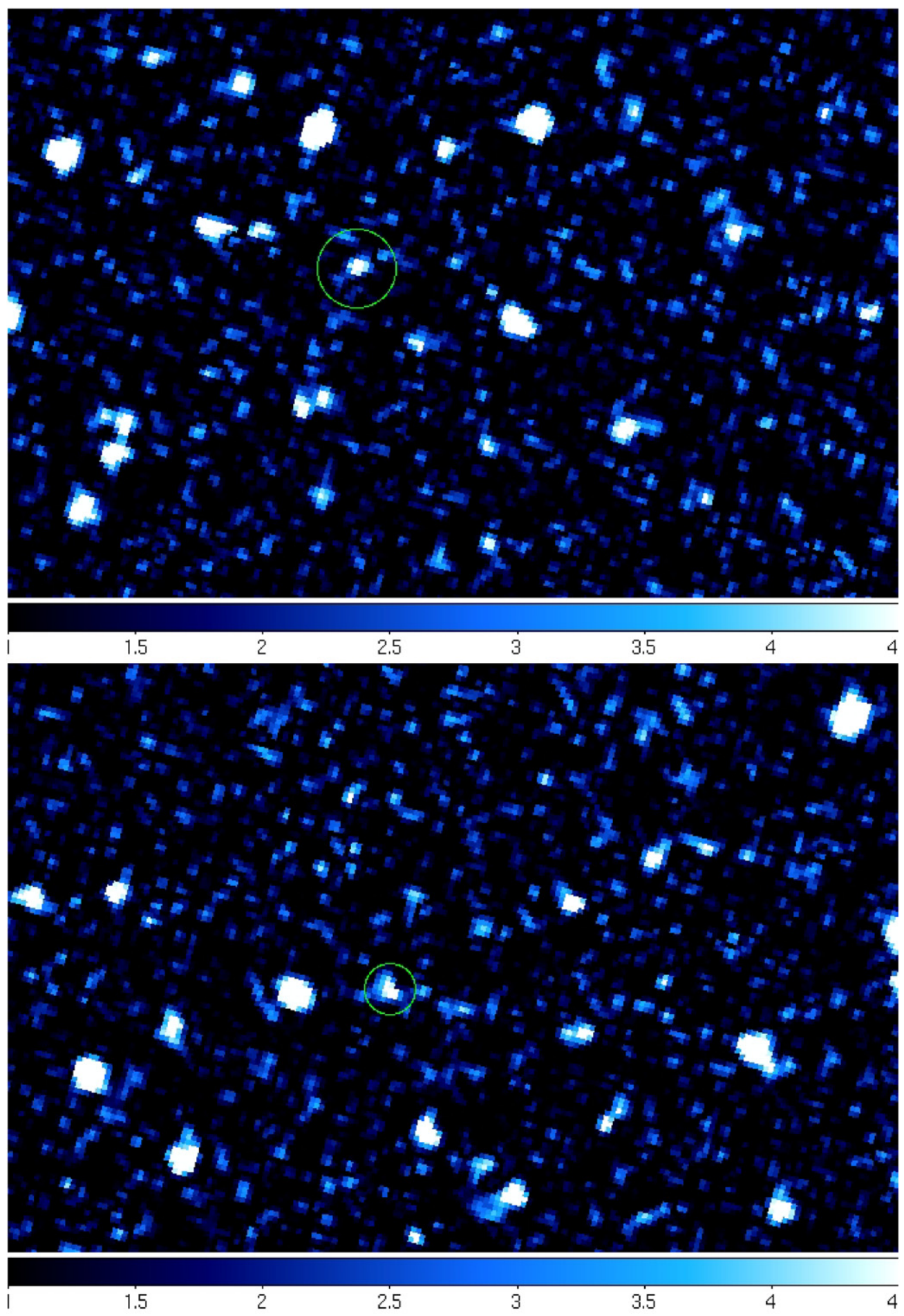

Figure 9. Signal-to-noise maps constructed from our reduced NGC 2362 MIPS mosaic image with the positions of ID-41 (top) and ID-63 (bottom) circled. The color stretch in each panel goes from $1 \sigma$ to $4.5 \sigma$ (black to blue to white). The signal to noise for the integrated flux is $\sim 5$ for both ID-41 and 63 . Luhman et al. (2010) claim "from inspection of the mosaic" that both of these sources have signal to noise ratio $(\mathrm{S} / \mathrm{N})<3$. If that were the case, none of the pixels centered on these targets would be light blue or white. However, their removal of two stars (IDs 663 and 1091 but not IDs 809 or 931) from the Irwin et al. sample not considered here is better justified: one does has a low signal to noise and the other is blended with a different source.

(A color version of this figure is available in the online journal.)

NGC 2362. For NGC 2362, we analyze the Spitzer IRAC and MIPS photometry presented by Currie et al. (2009). Currie et al. (2009) present data for two membership lists for NGC 2362: members/probable members identified by Dahm (2005) and candidate members identified by Irwin et al. (2008). Here, we analyze Spitzer data only for members/probable members identified by Dahm (2005) to be conservative. We include the two sources-IDs 41 and 63-not considered in analysis by Luhman et al. (2010), because both have $\approx 5 \sigma$ detections and thus should be included in our analysis (Figure 9).

Currie et al. (2009) already model the SEDs for NGC 2362 stars with the Robitaille et al. (2006) radiative transfer 
grid to identify disks with inner holes. Therefore, we only compare NGC 2362 SEDs through $24 \mu \mathrm{m}$ to the appropriate flat reprocessing disk models. As starting points for our flat disk comparisons, we use extinction estimates listed in Currie et al. (2009) that were based off of comparisons with the Kenyon \& Hartmann (1995) colors and off of output from fits with the Robitaille et al. models treating the extinction as a free parameter.

$\eta$ Cha. For $\eta$ Cha, we use the IRAC and MIPS $24 \mu \mathrm{m}$ and $70 \mu \mathrm{m}$ photometry and spectral types from Sicilia-Aguilar et al. (2009), MIPS $70 \mu \mathrm{m}$ upper limits and MIPS $160 \mu \mathrm{m}$ photometry/upper limits from Gautier et al. (2008), and IRS data presented in Bouwman et al. (2006) and Sicilia-Aguilar et al. (2009). To model $\eta$ Cha sources, we compare source SEDs to appropriate flat disk models and fit their SEDs through $160 \mu \mathrm{m}$ with the Robitaille model grid to identify inner holes and estimate disk masses.

\subsection{Classification Method}

Our disk classification method is an expanded, more thorough version of that used in Section 3. As in Section 3, we identify transitional disks with inner holes from fitting source SEDs with the Robitaille radiative transfer models. The difference between our method here and in Section 3 is in our differentiation between primordial disks and homologously depleted transitional disks. In Section 3, members of the latter group were identified as those having optically thin mid-IR emission (from comparing their emission to the flat disk limit) but lacking evidence for inner holes. As stated in Section 3, we considered the classification of these disks as provisional since weak midIR emission could be due to a low overall mass of submicron to submillimeter-sized dust or the growth and settling of just the smallest, submicron-sized grains to micron sizes or larger. Modeling based on 1-24 $\mu \mathrm{m}$ data alone is unable to break this degeneracy.

However, SED modeling of sources with far-IR/submillimeter data helps break this degeneracy (e.g., Wood et al. 2002). Taurus and $\eta$ Cha include many sources with far-IR and/or submillimeter data. Thus, SED modeling of Taurus and $\eta$ Cha sources can identify which ones with optically thin mid-IR emission but no inner hole have (1) a low inferred disk mass or (2) an inferred disk mass comparable to optically thick primordial disks. The former group are classified as homologously depleted transitional disks (Currie et al. 2009); the latter group could be primordial disks undergoing extreme grain growth/ dust settling/shadowing (Furlan et al. 2006).

To identify the range of disk masses for optically thick primordial disks and thus most appropriate limit for dividing between primordial disks and homologously depleted transitional disks, we refer to SED modeling results for Taurus (Andrews \& Williams 2005) which are discussed in detail in the Appendix. Briefly, typical primordial disk masses as a fraction of stellar mass are $M_{\text {disk }} \sim 0.01 M_{\star}$ for Taurus: the interquartile range covers $M_{\text {disk }} \sim 0.003-0.03 M_{\star}$ (see the Appendix, Figure 7; Andrews \& Williams 2007). ${ }^{7}$ About $92 \%-96 \%$ of the primordial disks in the Andrews \& Williams Taurus sample have inferred masses greater than $0.001 M_{\star}$ (cf. the Appendix). As discussed in the Appendix, far-IR data $(\lambda \gtrsim 70 \mu \mathrm{m})$ are sufficient to estimate the disk mass at a precision useful for our study.

\footnotetext{
7 Andrews \& Williams $(2005,2007)$ use the term "Class II objects" to describe sources with optically thick disk emission, though their sample includes some transitional disks with inner holes (e.g., UX Tau, CoKu Tau/4, GM Aur).
}

Thus, if we define the range of primordial disk masses by the interquartile range, the appropriate limit differentiating optically thick primordial disks from homologously depleted transitional disks is $M_{\text {disk }}=0.003 M_{\star}$. If we use the full range of primordial disk masses, the appropriate limit is $M_{\text {disk }}=$ $0.001 M_{\star}$. As discussed in the Appendix, these estimates for typical masses of optically thick primordial disks, and thus our criteria for separating primordial and transitional disks, are not compromised by incompleteness. In our analysis, the disk mass is the median value of masses from the best-fit Robitaille et al. (2006) models; to determine the fractional disk mass, we use the Baraffe (1998) isochrones and the effective temperature scales from Currie et al. (2010) for K5-M2 stars and Luhman et al. (2003) for later stars.

Combining our criteria from Section 3 with additional criteria presented in this section, we consider a disk in Taurus or $\eta$ Cha to be a primordial disk if it meets the following two conditions:

1. It lacks evidence for an inner hole/gap as inferred from SED modeling.

2. Its mid-IR emission exceeds the optically thick, flat disk limit $o r$ it has an inferred disk mass of $M_{d}>0.003 M_{\star}$.

Disks with inferred masses of greater than $0.001 M_{\star}$ and up to $0.003 M_{\star}$ but no inner hole are considered to be borderline cases, as their classification depends on whether the interquartile range or full range of optically thick primordial disk masses is used to separate primordial disks from homologously depleted transitional disks. Disks with masses $\leqslant 0.001 M_{\star}$, optically thin mid-IR emission, and no inner hole are classified as homologously depleted transitional disks. We then determine the transitional disk frequency by dividing the number of transitional disks by the number of transitional + primordial disks.

To analyze disks in clusters lacking far-IR/submillimeter data-IC 348 and NGC 2362-we first assign provisional classifications based on Section 3 methods, which yield provisional frequencies for transitional disks. Since mid-IR data alone are unable to differentiate between disks with a low dust mass and those with substantial dust settling, some the disks identified as transitional based on mid-IR data could instead be primordial disks. Thus, we use the Taurus results to identify the mid-IR colors of transitional disks and determine the "contamination rate" of the transitional disk population selected by mid-IR data alone. Transitional disk frequencies for IC 348 and NGC 2362 are then revised after taking into account this contamination rate.

\subsection{Transitional Disks in Taurus}

Modeling results for 25 Taurus members are summarized in Tables 6 and 7. Several of these members have disks with inner holes-e.g., UX Tau, LkCa 15, and J04333905+2227207. Many others-e.g., VY Tau-lack clear evidence for an inner hole but have emission falling below the flat, optically thick disk limit (Figure 10). They also have weak submillimeter emission (Table 7). The weak near-IR through submillimeter emission from these disks identifies a substantial depletion of the entire dust grain population from hot inner disk regions to cold outer disk regions. Nearly all sources $(94 \%)$ with $K_{s}-[8] \leqslant 1.75$ have emission weaker than the optically thick, flat disk limit.

\subsubsection{Best-estimated Masses of Disks in Taurus}

Figure 11 displays the SEDs and histogram distribution of masses from best-fit models for several disks that lack inner 
Table 6

Properties of Selected Taurus Sources

\begin{tabular}{|c|c|c|c|c|c|c|c|c|}
\hline Name & ST & $\begin{array}{c}M_{\star} \\
\left(M_{\odot}\right)\end{array}$ & $A_{V}$ & $A_{V}$ Range & $K_{s}-[8]$ & $K_{s}-[24]$ & $\tau_{\text {mid-IR }}$ & $\begin{array}{l}\text { Inner } \\
\text { Hole? }\end{array}$ \\
\hline UX Tau & K5 & 1.0 & 0.75 & $0.25-1.25$ & 1.56 & 5.42 & Thin & $\mathrm{y}$ \\
\hline V807 Tau & K5 & 1.0 & 1.4 & $0.9-1.9$ & 1.46 & 3.91 & Thin & $\mathrm{n}$ \\
\hline LkCa 15 & K5 & 1.0 & 1.2 & $0.7-1.7$ & 1.58 & 5.06 & Thin & $\mathrm{y}$ \\
\hline FY Tau & K5 & 1.0 & 5 & $4-6$ & 1.68 & 3.93 & Thin & $\mathrm{n}$ \\
\hline JH 112 & K6 & 0.95 & 3.4 & $2.72-4.08$ & 1.97 & 5.34 & Thick & $\mathrm{n}$ \\
\hline V836 Tau & K7 & 0.85 & 1.05 & $0.55-1.55$ & 1.76 & 4.77 & Thin & $\mathrm{n}$ \\
\hline DN Tau & M0 & 0.75 & 0.7 & $0.2-1.2$ & 1.99 & 5.00 & Thin & $\mathrm{n}$ \\
\hline VY Tau & M0 & 0.75 & 1.1 & $0.6-1.6$ & 1.53 & 4.35 & Thin & $\mathrm{n}$ \\
\hline GO Tau & M0 & 0.75 & 2 & $1.5-2.5$ & 1.85 & 4.93 & Thin & $\mathrm{n}$ \\
\hline IP Tau & M0 & 0.75 & 0.6 & $0.1-1.1$ & 1.68 & 4.74 & Thick & $\mathrm{n}$ \\
\hline HO Tau & M0.5 & 0.65 & 1.3 & $0.8-1.8$ & 1.90 & 4.79 & Thick & $\mathrm{n}$ \\
\hline DH Tau & M1 & 0.6 & 1.9 & $1.4-2.4$ & 1.28 & 4.79 & Thin & $?$ \\
\hline FX Tau & M1.5 & 0.57 & 2 & $1.5-2.5$ & 1.90 & 4.85 & Thick & $\mathrm{n}$ \\
\hline CY Tau & M1.5 & 0.57 & 1.7 & $1.2-2.2$ & 1.89 & 4.11 & Thick & $\mathrm{n}$ \\
\hline $\begin{array}{r}04333905 \\
+2227207\end{array}$ & M1.75 & 0.57 & 7.9 & $2.4-3.6$ & 1.49 & 5.81 & Thin & $\mathrm{y}$ \\
\hline JH 223 & M2 & 0.55 & 2.2 & $1.6-2.6$ & 1.72 & 4.27 & Thin & $\mathrm{n}$ \\
\hline GH Tau & M2 & 0.55 & 1 & $0.5-1.5$ & 1.79 & 4.51 & Thick & $\mathrm{n}$ \\
\hline ZZ Tau & M3 & 0.4 & 1.2 & $0.7-1.7$ & 1.39 & 3.91 & Thin & $\mathrm{n}$ \\
\hline XEST13-10 & M3 & 0.4 & 6 & $4.8-7.2$ & 1.52 & 4.40 & Thin & $\mathrm{n}$ \\
\hline CIDA-8 & M3.5 & 0.3 & 2.25 & $1.75-2.75$ & 1.49 & 4.30 & Thin & $\mathrm{n}$ \\
\hline $\begin{array}{r}04231822 \\
+2641156\end{array}$ & M3.5 & 0.3 & 8 & $6.4-9.6$ & 1.53 & 5.11 & Thin & $\mathrm{n}$ \\
\hline $\begin{array}{r}04153916 \\
+2818586\end{array}$ & M3.75 & 0.275 & 2 & $1.5-2.5$ & 1.62 & 4.93 & Thin & $\mathrm{n}$ \\
\hline FP Tau & M4 & 0.25 & 0.5 & $0-1$ & 1.52 & 4.53 & Thin & $\mathrm{n}$ \\
\hline ITG-15 & M5 & 0.175 & 3.25 & $2.6-3.9$ & 1.62 & 4.79 & Thin & $\mathrm{n}$ \\
\hline $\begin{array}{r}04202555 \\
+2700355\end{array}$ & M5.25 & 0.15 & 2.1 & $1.6-2.6$ & 1.69 & 5.37 & Thin & $?$ \\
\hline
\end{tabular}

Notes. The $K_{s}-[8]$ and $K_{s}-[24]$ colors listed are dereddened colors as determined by Luhman et al. (2010). The stellar masses are computed using the T. Currie et al. (2011, in preparation) effective temperature scale for K5-M2 stars and the Luhman et al. (2003) scale for later stars and the Baraffe (1998) isochrones. $A_{V}$ was determined individually by fitting the optical-to-near-IR SED for stellar photospheres using the T. Currie et al. (2011, in preparation) intrinsic colors for a given effective temperature and using the T. Currie et al. (2011, in preparation) and Luhman et al. (2003) effective temperature scales. The range in $A_{V}$ assumes a nominal $20 \%$ extinction uncertainty with a minimum uncertainty of $0.5 \mathrm{mag}$.

holes but have weak near-to-mid-IR emission-VY Tau, JH 223, ZZ Tau, and FP Tau-and indicates that they have low inferred masses $\left(M_{\text {disk }} \leqslant 0.001 M_{\star}\right)$. Such sources fit our criteria for being a homologously depleted transitional disk. Several others (e.g., V807 Tau) lacking inner holes have optically thin near-to-mid-IR emission and $M_{\text {disk }}=0.001-0.003 M_{\star}$ : these are borderline cases. The intrinsic sampling of the Robitaille grid is heavily weighted toward disk models with masses $\sim 0.001-0.01 M_{\odot}$ (shaded region). In spite of this bias, the inferred disk masses for sources like those in Figure 11 are at least an order of magnitude lower.

Mass estimates based on submillimeter data alone (Andrews $\&$ Williams 2005) provide a simple but independent check on the estimates derived from radiative transfer modeling. All of the homologously depleted transitional disks with submillimeter data that are listed in Table 7 have non-detections at $350-850 \mu \mathrm{m}$, and thus their masses have a wide range of possible values. However, the submillimeter-derived upper limits constrain the mass of optically thin emitting dust, which is useful since all of these sources have optically thin near-to-mid-IR dust emission and optical depth decreases with increasing wavelength. For the homologously depleted disks, submillimeter-derived upper limits range from $5.5 \times 10^{-4} M_{\star}$ to $10^{-3} M_{\star}$. In other words, submillimeter data alone provide evidence that these disks are less massive than primordial disks.

\subsubsection{Frequency of Transitional Disks in Taurus}

To compute the relative frequency of transitional disks around K5-M6 stars, we combine the transitional disk population identified by Luhman et al. (2010) and newly identified transitional disks from Table 7. For the primordial disk population, we consider all Taurus members with Spitzer photometry that are not Class I protostars. Based on mid-IR colors, Luhman et al. (2010) identified 18 transitional disks around K5-M6 stars: nine around K5-M2 stars and nine around later stars. To these, we add 6-7 transitional disks around K5-M2 stars and 3-7 transitional disks around later stars identified from SED modeling.

Combining both transitional disk samples, the frequency of transitional disks is $0.19-0.22(27-32 / 145)$ for the entire population, $0.20-0.21(15-17 / 77)$ for K5-M2 stars, and 0.18-0.24 (12-16/68) for M3-M6 stars. Two transitional disks identified by Luhman et al. (2010) have extremely tenuous $24 \mu \mathrm{m}$ excess emission $\left(K_{s}-[24] \sim 0.5-1\right)$ and could be young debris disks (Carpenter et al. 2009). If we remove these sources, the transitional disk frequency for the entire population drops to 0.18 and 0.22 , while the frequency for K5-M2 stars drops to $\sim 0.16-0.18$. Thus, we find a slightly higher percentage of transitional disks around K5-M6 stars than Luhman et al. (2010) find for K5-M5 stars $(\sim 20 \%$ versus their $13 \%(15 / 113))$. 
Table 7

Disk Evolutionary States for Selected Taurus Sources

\begin{tabular}{|c|c|c|c|c|c|c|c|c|}
\hline Name & $M_{\text {disk }}$ & $\frac{M_{\text {disk }}}{M_{\star}}$ & $\begin{array}{c}\frac{M_{\text {disk }}}{M_{\star}} \\
(\mathrm{IQR})\end{array}$ & $f\left(<1.3 \times 10^{-3}\right)$ & $\begin{array}{c}\text { Submm. } \\
\text { Ref. }\end{array}$ & $\begin{array}{c}M_{\text {disk }} \\
\left(M_{\odot, \text { submm }}\right)\end{array}$ & $\begin{array}{c}\frac{M_{\text {disk }}}{M_{\star}} \\
\text { (submm) }\end{array}$ & Disk Class \\
\hline UX Tau & 0.02 & 0.02 & {$[0.017,0.022]$} & 0,0 & 1 & $5.1 \times 10^{-3}$ & $5.1 \times 10^{-3}$ & $\mathrm{TD}(\mathrm{IH})$ \\
\hline V807 Tau & $3 \times 10^{-3}$ & $3 \times 10^{-3}$ & {$\left[3 \times 10^{-3}, 4 \times 10^{-3}\right]$} & $0,0.5$ & 1 & $10^{-3}$ & $10^{-3}$ & $\mathrm{PD} / \mathrm{TD}(\mathrm{HD})$ \\
\hline LkCa 15 & 0.01 & 0.01 & {$[0.01,0.025]$} & 0,0 & 1 & 0.05 & 0.05 & $\mathrm{TD}(\mathrm{IH})$ \\
\hline FY Tau & $10^{-4}$ & $10^{-4}$ & {$\left[6 \times 10^{-6}, 1 \times 10^{-3}\right]$} & $0.76,0.82$ & 1 & $7 \times 10^{-4}$ & $7 \times 10^{-4}$ & $\mathrm{TD}(\mathrm{HD})$ \\
\hline JH 112 & $8 \times 10^{-3}$ & $9.4 \times 10^{-3}$ & {$\left[4 \times 10^{-3}, 0.01\right]$} & $0.06,0.12$ & 1 & $10^{-3}$ & $1.05 \times 10^{-3}$ & PD \\
\hline V836 Tau & 0.01 & 0.012 & {$[0.012,0.014]$} & 0,0 & 1 & 0.01 & 0.012 & PD \\
\hline DN Tau & 0.01 & 0.013 & {$[0.013,0.03]$} & 0,0 & 1,4 & $3 \times 10^{-2}$ & $4 \times 10^{-2}$ & PD \\
\hline VY Tau & $2 \times 10^{-4}$ & $2.7 \times 10^{-4}$ & {$\left[3.4 \times 10^{-5}, 9 \times 10^{-4}\right]$} & $0.76,0.91$ & 1 & $<5 \times 10^{-4}$ & $<6.7 \times 10^{-4}$ & $\mathrm{TD}(\mathrm{HD})$ \\
\hline GO Tau & $6 \times 10^{-3}$ & $8 \times 10^{-3}$ & {$\left[5 \times 10^{-3}, 0.023\right]$} & 0,0 & 1 & 0.07 & 0.09 & PD \\
\hline IP Tau & $8 \times 10^{-3}$ & 0.011 & {$\left[7 \times 10^{-3}, 0.016\right]$} & $0,0.05$ & 1 & $3 \times 10^{-3}$ & $4 \times 10^{-3}$ & PD \\
\hline HO Tau & $8 \times 10^{-3}$ & 0.012 & {$[0.013,0.013]$} & 0,0 & 1 & $2 \times 10^{-3}$ & $3.1 \times 10^{-3}$ & $\mathrm{PD}$ \\
\hline DH Tau & $5 \times 10^{-3}$ & 0.01 & {$[0.01,0.018]$} & $0,0.27$ & 1 & $3 \times 10^{-3}$ & $5 \times 10^{-3}$ & $\mathrm{PD} / \mathrm{TD}(\mathrm{IH})$ \\
\hline FX Tau & $4 \times 10^{-3}$ & $7 \times 10^{-3}$ & {$\left[5 \times 10^{-3}, 0.013\right]$} & $0.09,0.22$ & 1 & $9 \times 10^{-4}$ & $1.5 \times 10^{-3}$ & $\mathrm{PD}$ \\
\hline CY Tau & $8 \times 10^{-3}$ & 0.014 & {$[0.014,0.025]$} & 0,0 & 1,4 & $6 \times 10^{-3}$ & 0.015 & PD \\
\hline $\begin{array}{r}04333905 \\
+2227207\end{array}$ & $4 \times 10^{-4}$ & $7 \times 10^{-4}$ & {$\left[1.7 \times 10^{-4}, 2.7 \times 10^{-3}\right]$} & $0.63,0.68$ & $\ldots$ & $\ldots$ & $\ldots$ & $\mathrm{TD}(\mathrm{IH})$ \\
\hline JH 223 & $7.9 \times 10^{-5}$ & $1.4 \times 10^{-4}$ & {$\left[8.5 \times 10^{-5}, 1 \times 10^{-3}\right]$} & $0.75,0.82$ & 1 & $<3 \times 10^{-4}$ & $<5.5 \times 10^{-4}$ & $\mathrm{TD}(\mathrm{HD})$ \\
\hline GH Tau & $4.1 \times 10^{-3}$ & $7.5 \times 10^{-3}$ & {$\left[4 \times 10^{-3}, 0.01\right]$} & $0.03,0.16$ & 1 & $7 \times 10^{-4}$ & $1.3 \times 10^{-3}$ & PD \\
\hline ZZ Tau & $1 \times 10^{-4}$ & $2.5 \times 10^{-4}$ & {$\left[2 \times 10^{-5}, 8 \times 10^{-4}\right]$} & $0.77,0.86$ & 1,3 & $<4 \times 10^{-4}$ & $<10^{-3}$ & $\mathrm{TD}(\mathrm{HD})$ \\
\hline XEST13-10 & $4 \times 10^{-4}$ & $1 \times 10^{-3}$ & {$\left[2.5 \times 10^{-4}, 3 \times 10^{-3}\right]$} & $0.5,0.72$ & $\ldots$ & $\ldots$ & $\ldots$ & $\mathrm{TD}(\mathrm{HD})$ \\
\hline CIDA-8 & $8 \times 10^{-3}$ & 0.03 & {$[0.03,0.04]$} & 0,0 & 1 & $10^{-3}$ & $3.3 \times 10^{-3}$ & PD \\
\hline $\begin{array}{l}04231822 \\
+2641156\end{array}$ & $5 \times 10^{-4}$ & $1.7 \times 10^{-3}$ & {$\left[3 \times 10^{-4}, 5 \times 10^{-3}\right]$} & $0.43,0.66$ & $\ldots$ & $\ldots$ & $\ldots$ & $\mathrm{PD} / \mathrm{TD}(\mathrm{HD})$ \\
\hline $\begin{array}{r}04153916 \\
+2818586\end{array}$ & $4 \times 10^{-4}$ & $1.5 \times 10^{-3}$ & {$\left[4 \times 10^{-4}, 6 \times 10^{-3}\right]$} & $0.39,0.59$ & . & . & $\ldots$ & $\mathrm{PD} / \mathrm{TD}(\mathrm{HD})$ \\
\hline FP Tau & $2.5 \times 10^{-4}$ & $1 \times 10^{-3}$ & {$\left[4 \times 10^{-4}, 4 \times 10^{-3}\right]$} & $0.53,0.68$ & 2 & $<1.3 \times 10^{-4}$ & $<5.2 \times 10^{-4}$ & $\mathrm{TD}(\mathrm{HD})$ \\
\hline ITG-15 & $3 \times 10^{-4}$ & $1.7 \times 10^{-3}$ & {$\left[3.6 \times 10^{-4}, 9 \times 10^{-3}\right]$} & $0.49,0.65$ & $\ldots$ & $\ldots$ & $\ldots$ & $\mathrm{PD} / \mathrm{TD}(\mathrm{HD})$ \\
\hline $\begin{array}{r}04202555 \\
+2700355\end{array}$ & $4 \times 10^{-4}$ & $2.7 \times 10^{-3}$ & {$\left[1 \times 10^{-3}, 3 \times 10^{-3}\right]$} & $0.07,0.53$ & $\ldots$ & $\ldots$ & $\ldots$ & $\mathrm{PD} / \mathrm{TD}(\mathrm{IH})$ \\
\hline
\end{tabular}

Notes. IQR refers to the interquartile range of fractional disk masses from best-fit models. The identification "Lu10" refers to Luhman et al. (2010), where the classification listed is based on applying the Luhman et al. (2010) color criteria. The references for submillimeter data and (sub)millimeter-derived disk masses are the following: (1) Andrews \& Williams 2005, (2) Jewitt 1994, (3) Jensen et al. 1994, and (4) Beckwith \& Sargent 1991. The fractional disk masses are computed using the T. Currie et al. (2011, in preparation) effective temperature scale for K5-M2 stars and the Luhman et al. (2003) scale for later stars and the Baraffe (1998) isochrones. Sources with disk evolutionary states listed as PD/TD(IH) or PD/TD(HD) have uncertain states: either SED modeling is inconclusive as to whether the disks likely have inner holes $(\mathrm{PD} / \mathrm{TD}(\mathrm{IH}))$ or the classification depends on whether the full range or interquartile range of disk masses is used to identify primordial disks (PD/TD(HD)). Luhman et al. (2010) label all of these disks as primordial disks.

\subsubsection{Mid-IR Colors of Primordial Disks and Transitional Disks}

To guide our analysis of other clusters lacking farIR/submillimeter data (e.g., IC 348 and NGC 2362), we determine the mid-IR colors $\left(K_{s}-[8]\right)$ that identify primordial disks and transitional disks. Figure 12 plots the mid-IR colors of Taurus members separated by disk evolutionary state. Only members with primordial disks (black circles) have $K_{s}-[8]>1.75$. Taurus members with $K_{s}-[8]$ bluer than the horizontal dashed line $\left(K_{s}-[8]=1.25\right)$ do not have primordial disks. Members with intermediate colors include primordial disks, transitional disks (gray circles enclosed by squares), and borderline cases (black circles enclosed by squares). Thus, there are two regions of mid-IR color-color space containing transitional disks: an "uncontaminated" region with blue colors consisting only of transitional disks and a "contaminated" region with redder colors which includes both transitional disks and primordial disks.

Comparing the number of transitional disks and primordial disks in the "contaminated" color region for Taurus members with far-IR/submillimeter data provides an estimate for the level of contamination in other clusters lacking far-IR/submillimeter data. As shown by Figure 12 and Table 6, there are nine K5-M2 members with $K_{s}-[8]=1.25-1.75$ : six sources identified as transitional disks, two identified as primordial disks, and one borderline case (V807 Tau). Thus, 22\%-33\% (2-3/9) of the sources with $K_{s}-[8]=1.25-1.75$ and provisionally identified as transitional disks from mid-IR data alone could in fact be primordial disks with substantial dust settling. For M3-M6 stars with $K_{s}-[8]=1.25-1.75$, three sources listed in Table 7 plus two identified by Luhman et al. (2010)—J04330945 and J04213459-are transitional disks, one is a primordial disk, and four are borderline cases. Thus, the contamination rate for M3-M6 stars with $K_{s}-[8]=1.25-1.75$ is $10 \%-50 \%(1-5 / 10)$.

\subsubsection{Incorporating Uncertainties in Disk Mass Estimates}

Here, we quantitatively assess how the intrinsic uncertainty in disk mass estimates affects our conclusions about the frequency of transitional disks in Taurus and the contamination level from selecting transitional disks by mid-IR colors. First, we compare the interquartile range of disk masses from best-fit models for each star. Figure 13 shows that the range of disk masses for homologously depleted disks and primordial disks are distinct (see also Table 6). The upper quartile of disk masses for homologously depleted disks ranges from $\sim 9 \times 10^{-4} M_{\star}$ to $4 \times 10^{-3} M_{\star}$. The lower quartile of masses for primordial disks 

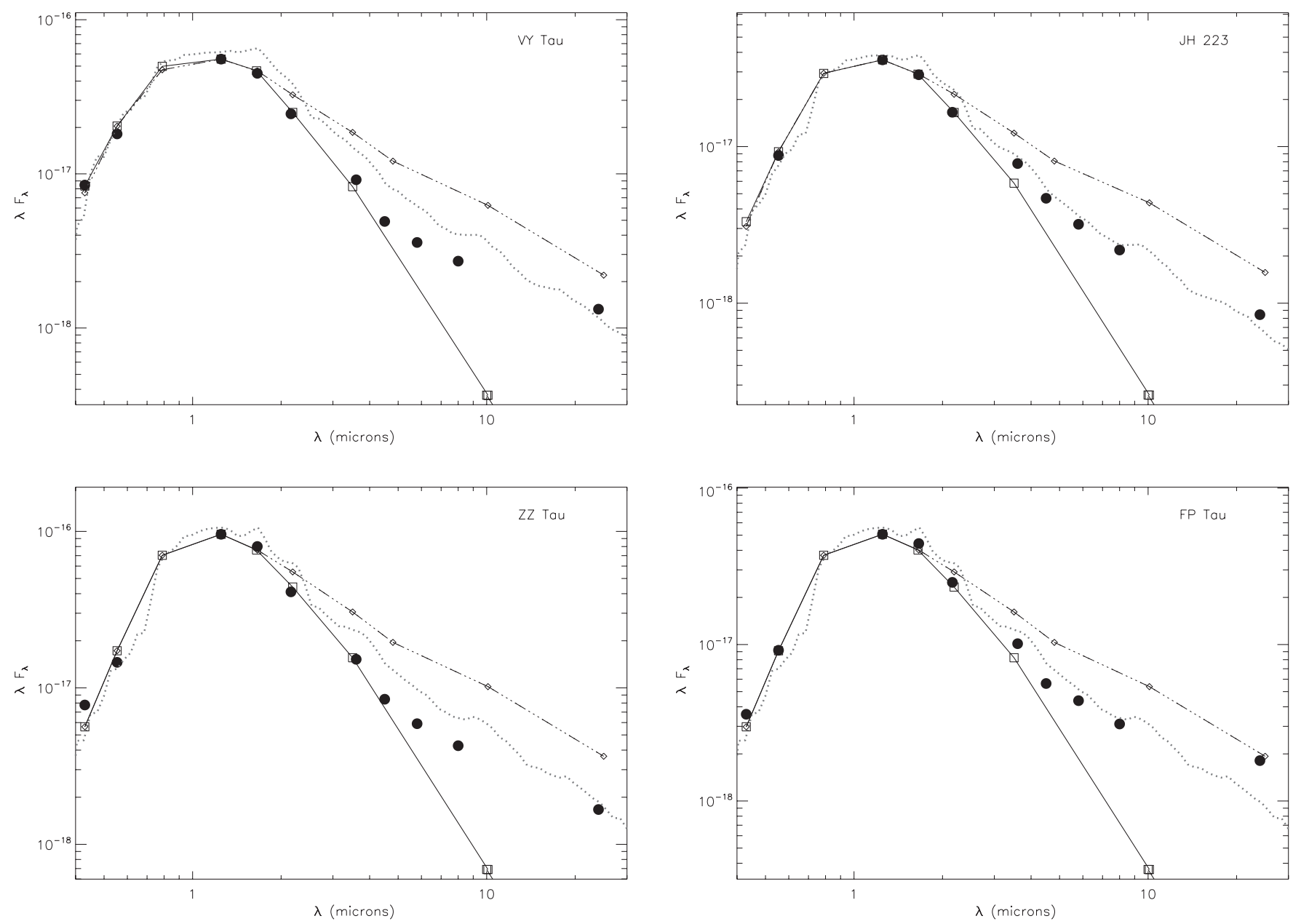

Figure 10. SEDs for four homologously depleted transitional disks in Taurus, showing optical-to-mid-IR photometric data compared to the flat, optically thick reprocessing disk limit and the lower-quartile Taurus SED.

is systematically larger and does not overlap, ranging from $4 \times$ $10^{-3} M_{\star}$ to $10^{-2} M_{\star}$. The masses of disks in borderline cases are also systematically low, though the upper quartiles for three of the five stars-J04231822+264116, J04153916+2818586, and ITG-15-overlap slightly with the lower-quartile masses for several primordial disks (e.g., GH Tau).

Second, we consider the full distribution of disk masses from best-fit models for each star to identify the fraction of models yielding $M_{\text {disk }} / M_{\star} \leqslant 0.001$ and $0.003\left(f<1,3 \times 10^{-3}\right)$, our thresholds identifying homologously depleted transitional disks and borderline cases. Our goal here is to derive a purely probabilistic estimate of the transitional disk population from Table 6 to combine with the transitional disks listed by Luhman et al. (2010) and then to compare with previous estimates of the total population in this section. Here, the number of transitional disks around $n$ stars listed in Table 6 each with $f\left(\mathrm{TD}_{i}\right)$ of their disk models fulfilling our definition of a transitional disk is

$$
n(\mathrm{TD})=\sum_{\mathrm{i}=0}^{\mathrm{n}} \mathrm{f}\left(\mathrm{TD}_{\mathrm{i}}\right)
$$

For disks with inner holes-e.g., LkCa 15-determined by imaging and SED modeling $f\left(\mathrm{TD}_{i}\right)=1$, otherwise it equals $f\left(<1,3 \times 10^{-3}\right)$ as listed in Table 6. This exercise yields $n(\mathrm{TD})=10.6-13.8$ or $\sim 11-14(n(\mathrm{TD})=5.5-7.1$ for $\mathrm{K} 5-\mathrm{M} 2$ stars, 5.2-6.7 for M3-M6 stars).
Combining our probabilistic estimate for the transitional disks population drawn from Table 6 and the 18 transitional disks identified by Luhman et al. (2010) yields total population of 29-32 (18+[11, 14]), in agreement with estimates derived in Section 4.3.2. The estimates divided by spectral-type range are likewise similar: $15-16(9+[6,7])$ for K5-M2 stars and 14-16 $(9+[5,7])$ for M3-M6 stars. Finally, the resulting contamination estimates agree well with previous ones: $22 \%-33 \%$ (2-3/9) for K5-M2 stars and $10 \%-30 \%(1-3 / 10)$ for M3-M6 stars. ${ }^{8}$

\subsection{Frequency of Transitional Disks in IC 348}

Table 8 summarizes our modeling results for selected IC 348 members; SEDs for members representing a range of mid-IR disk emission and provisional evolutionary states are shown in Figure 14. As with Taurus, we find that all but one of the members with $K_{s}-[8] \leqslant 1.75$ have optically thin mid-IR disk emission. Since our Taurus analysis finds that most members with these colors have low disk masses or inner holes (29-32/ 35 ), we infer that most IC 348 members with these colors also have low disk masses or inner holes. Of the 25 IC 348 stars

\footnotetext{
8 For K5-M2 stars, there are nine sources within our contamination region, our exercise predicts that $\sim 6-7$ are transitional disks, so the contamination rate is 2-3/9. For M3-M6 stars, there are 10 sources within the contamination region. Two of these are identified as transitional disks from Luhman et al. (2010), whereas our exercise predicts that 5-7 out of the eight others are transitional disks. Thus, the contamination rate is $1-3 / 10$.
} 

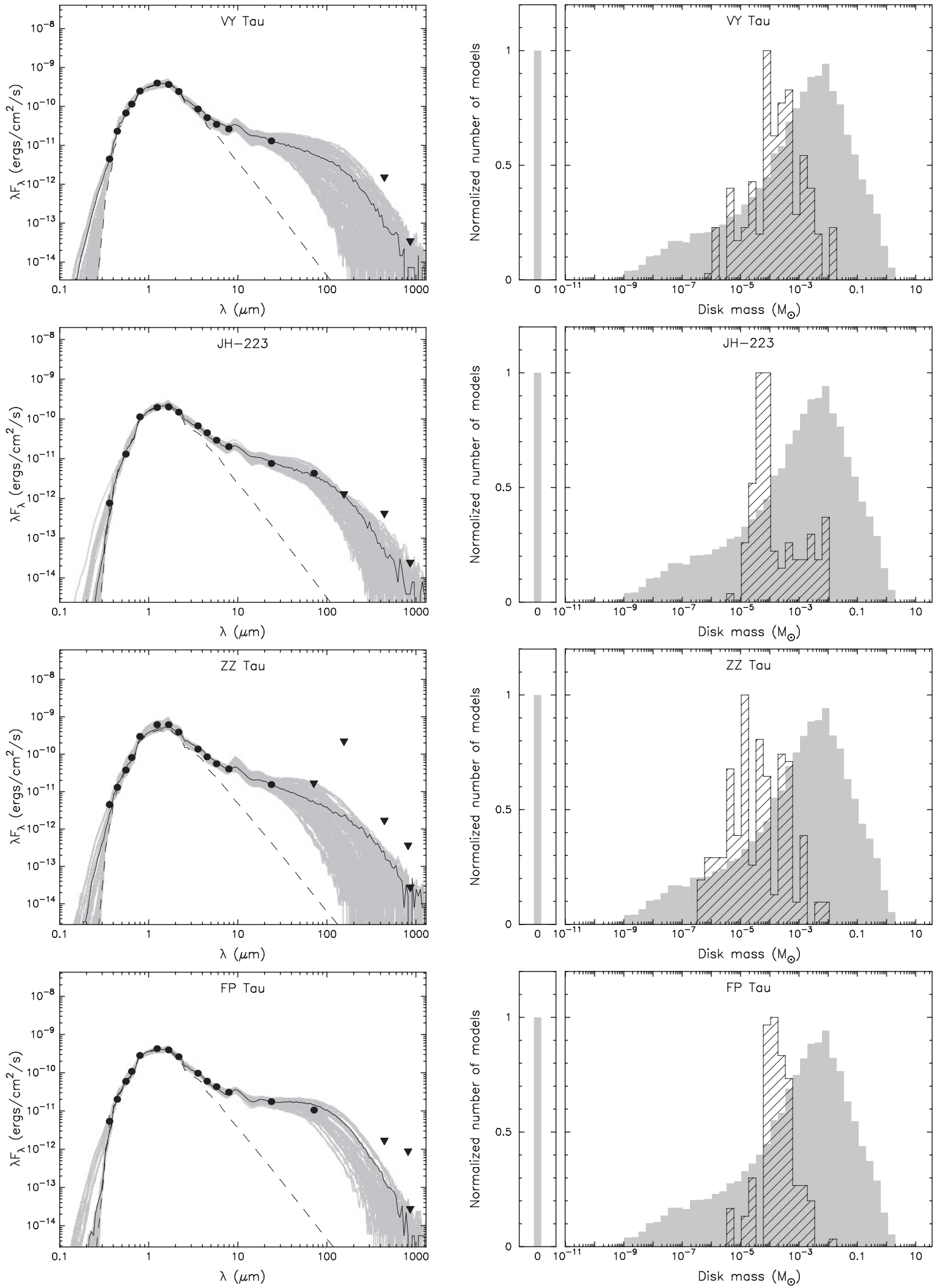

Figure 11. SED modeling of sources shown in previous figure, displaying the best-fit SEDs from the Robitaille grid along with the corresponding masses for the best-fit models. For the right-hand panels, the hatched region shows the normalized distribution of masses from best-fit models; the shaded gray region shows the normalized distribution of masses from all available models. The $x$-axis of the right-hand side figures lists the disk mass, not the fractional disk mass. The division corresponding to $M_{\text {disk }} / M_{\star}=10^{(-3)}$ for VY Tau, JH 223, ZZ Tau, and FP Tau occurs at $M_{\text {disk }}=7.5 \times 10^{-4}, 5.5 \times 10^{-4}, 4 \times 10^{-4}$, and $2.5 \times 10^{-4}$, respectively 



Figure 12. $K_{s}-[8]$ vs. $K_{s}-[24]$ colors for K5-M2 (left panel) and M3-M6 (right panel) Taurus members with data from Luhman et al. (2010) and Rebull et al. (2010). Gray circles surrounded by squares represent transitional disks identified from SED modeling, black dots represent primordial disks identified from SED modeling, and black dots surrounded by squares identify disks that could either be primordial or transitional. SED modeling was not performed for members represented as gray dots only. Sources between the horizontal dashed line $\left(K_{s}-[8]=1.25\right)$ and the horizontal dotted line $\left(K_{s}-[8]=1.75\right)$ include both primordial disks and transitional disks. Those above the horizontal dotted line include only primordial disks while primordial disks are absent below the horizontal dashed line.

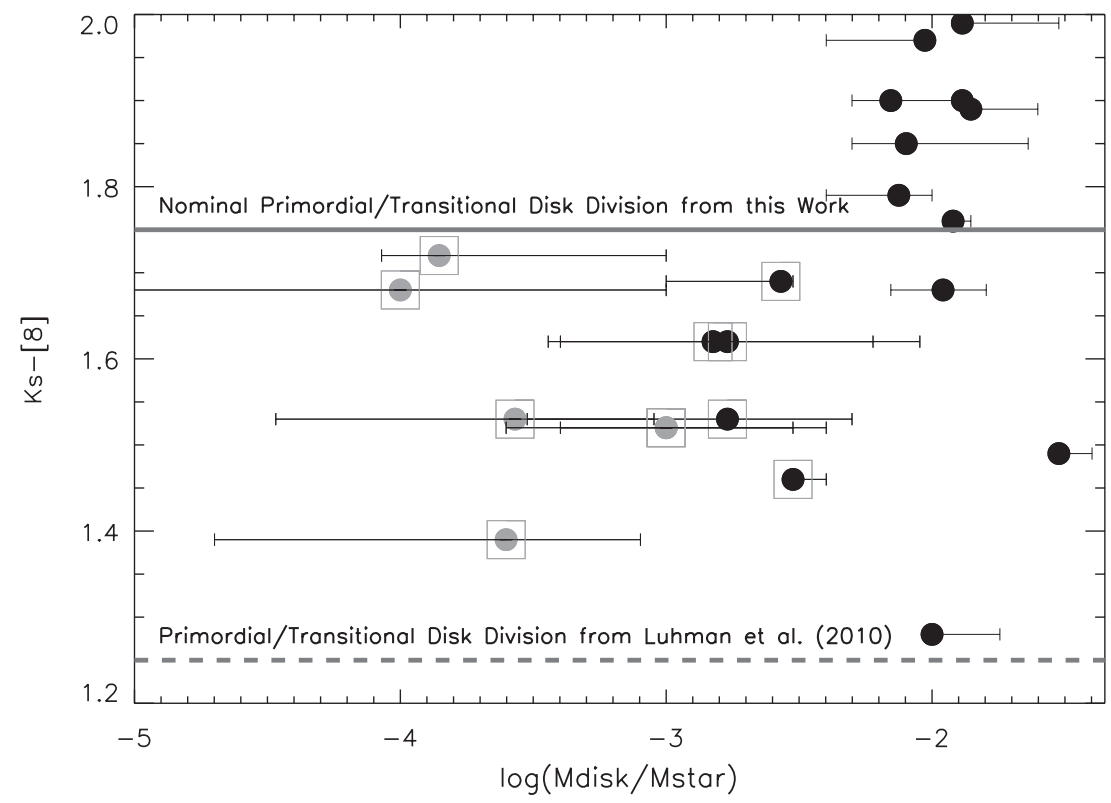

Figure 13. $K_{s}-[8]$ vs. fractional disk mass for homologously depleted transitional disks (gray circles surrounded by squares), "borderline" cases (black dots surrounded by squares), and primordial disks (black circles). The error bars identify the interquartile range of disk masses for each star. For reference, we show the threshold in $K_{s}-[8]$ color below which the Taurus transitional disk population begins (horizontal gray line, $K_{s}-[8]=1.75$ ) and that assumed in Luhman et al. $(2010)$ for K5-M2 stars (horizontal black dotted line, $K_{s}-[8]=1.25$ ). This plot shows that the ranges of disk masses for the homologously depleted transitional disk and primordial disk populations are distinct. The interquartile range of disk masses for the borderline cases slightly overlaps with the range for primordial disks.

modeled, 17 are consistent with being transitional disks based on 1-24 $\mu \mathrm{m}$ data, while eight are primordial disks.

To determine the frequency of transitional disks in IC 348, we use contamination estimates based on mid-IR colors from Taurus. Following the Taurus results, we adopt $K_{s}-[8]=1.75$ as our fiducial division between primordial disks and transitional disks and derive a provisional frequency for transitional disks. Then we derive a revised frequency taking into account contamination by primordial disks with $K_{s}-[8]=1.25-1.75$.

Based on this method, 8/33 K5-M2 stars and 43/81 M2.5-M6 stars have evidence for a disk with $K_{s}-[8]<1.75$, and thus fit our nominal near-to-mid-IR transitional disk criteria. Of the 8 (44) disks around K5-M2.5 (M2.5-M6) stars provisionally classified as transitional, 4 (19) have mid-IR colors within the "contaminated" region. Thus, corrected for contamination, about 32\%-40\% of K5-M6 IC 348 disks are transitional disks. The transitional disk population is heavily weighted toward later-type stars: $18 \%-24 \%$ of disks around K5-M2 stars have transitional disks $(6 / 33$ to $8 / 33)$, while $\sim 42 \%-51 \%$ of M2.5-M6 stars have transitional disks (34/81 to 41/81).

\subsection{Frequency of Transitional Disks in NGC 2362}

Table 9 summarizes our modeling results for NGC 2362. Near-to-mid-IR emission from most cluster members lies well below the optically thick, flat reprocessing disk limit, indicating that the mid-IR emission is at least marginally optically thin $(\tau \lesssim 1)$. Comparing this modeling to radiative transfer modeling performed for NGC 2362 in Currie et al. (2009) and 

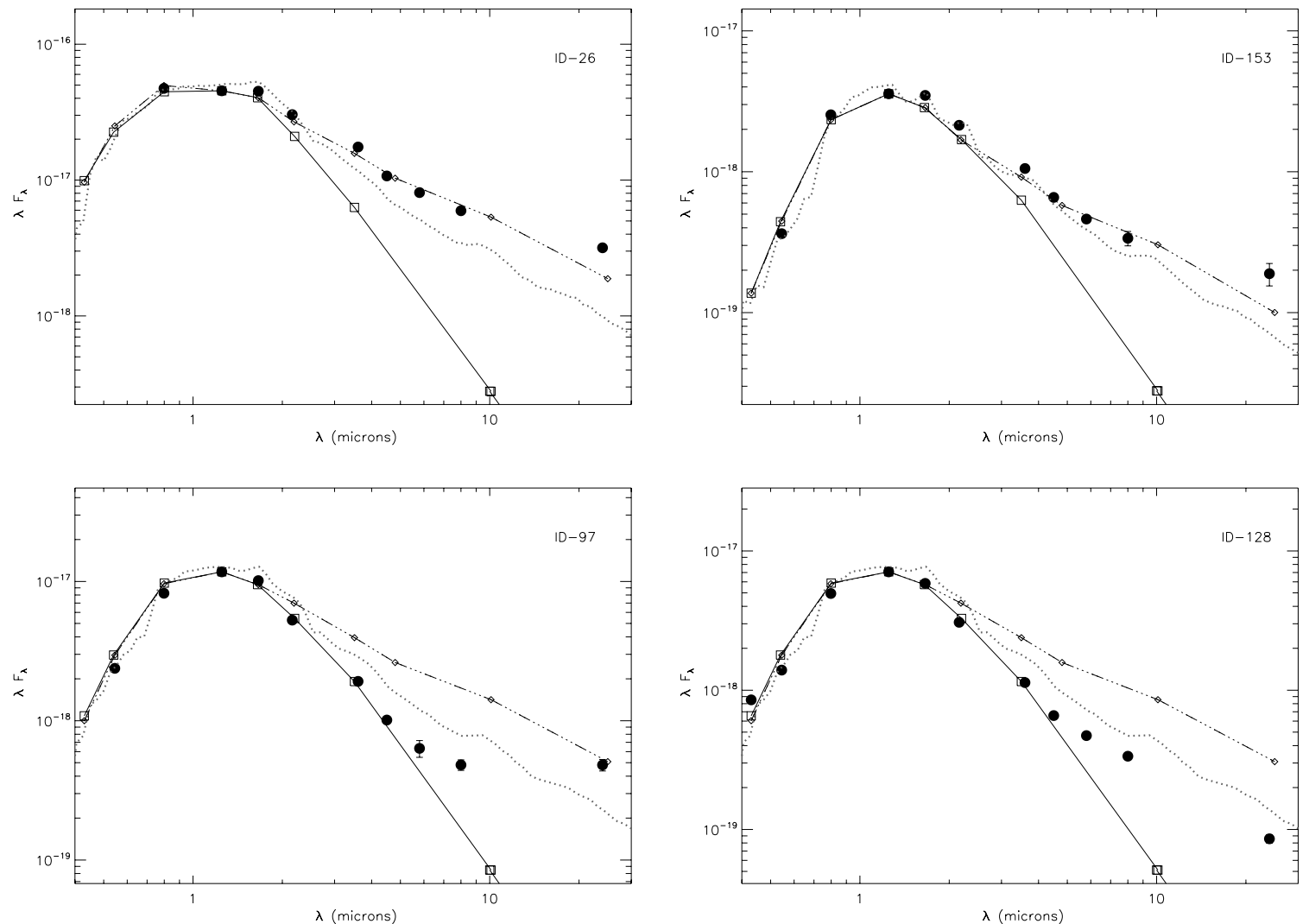

Figure 14. Representative SEDs for IC 348 sources. The provisional disk states for these members are (clockwise from top left): primordial disk, primordial disk, homologously depleted transitional disk, and transitional disk with an inner hole.

Table 8

Provisional Disk Evolutionary States for Selected Late-Type IC 348 Members Based on 1-24 $\mu \mathrm{m}$ Data

\begin{tabular}{|c|c|c|c|c|c|c|c|c|c|}
\hline ID & ST & $A_{v}($ Best $)$ & $K_{s}-[8]$ & $K_{s}-[24]$ & $\tau_{\text {mid-IR }}$ & Inner Hole? & CK09 & $\begin{array}{c}\text { Disk State Classification } \\
\text { Lu10 }\end{array}$ & This Work \\
\hline 21 & K0 & 5.4 & 1.03 & 5.43 & thin & $\mathrm{y}$ & $\mathrm{TD}(\mathrm{IH})$ & TD & $\mathrm{TD}(\mathrm{IH})$ \\
\hline 26 & K7 & 8 & 2.03 & 4.90 & thick & $\mathrm{n}$ & PD & PD & PD \\
\hline 32 & K7 & 4.6 & 1.95 & 4.81 & thick & $\mathrm{n}$ & PD & PD & PD \\
\hline 40 & K8 & 4 & 1.58 & 5.68 & thin & $?$ & PD & PD & $\mathrm{TD}(\mathrm{HD}) / \mathrm{TD}(\mathrm{IH})$ \\
\hline 55 & M0.5 & 11 & 1.93 & 4.86 & thick & $\mathrm{n}$ & PD & PD & PD \\
\hline 58 & M1.25 & 3.7 & 1.81 & 5.81 & thin & $?$ & PD & PD & $\mathrm{TD}(\mathrm{HD}) / \mathrm{TD}(\mathrm{IH}) ?$ \\
\hline 67 & M0.75 & 1.2 & 1.14 & 6.12 & thin & $\mathrm{y}$ & $\mathrm{TD}(\mathrm{IH})$ & TD & $\mathrm{TD}(\mathrm{IH})$ \\
\hline 68 & M3.5 & 2.25 & 1.43 & 4.14 & thin & $?$ & PD & PD & $\mathrm{TD}(\mathrm{HD}) / \mathrm{TD}(\mathrm{IH}) ?$ \\
\hline 72 & $\mathrm{M} 2.5$ & 1.7 & 0.75 & 5.65 & thin & $\mathrm{y}$ & $\mathrm{TD}(\mathrm{IH})$ & TD & $\mathrm{TD}(\mathrm{IH})$ \\
\hline 76 & M3.75 & 3.0 & 1.48 & 4.41 & thin & $\mathrm{n}$ & PD & TD & $\mathrm{TD}(\mathrm{HD})$ \\
\hline 97 & M2.25 & 5.5 & 1.30 & 4.85 & thin & y & $\mathrm{PD}$ & PD & $\mathrm{TD}(\mathrm{IH})$ \\
\hline 110 & M2 & 5.0 & 1.62 & 5.90 & thin & $\mathrm{y}$ & PD & PD & $\mathrm{TD}(\mathrm{IH})$ \\
\hline 128 & M2 & 2.75 & 1.47 & 3.54 & thin & $\mathrm{n}$ & $\mathrm{PD}$ & PD & $\mathrm{TD}(\mathrm{HD})$ \\
\hline 129 & M2 & 2.0 & 1.88 & 4.32 & thin & $\mathrm{n}$ & PD & PD & $\mathrm{TD}(\mathrm{HD})$ \\
\hline 135 & M4.5 & 0.9 & 1.41 & 3.48 & thin & $\mathrm{n}$ & PD & TD & TD(HD) \\
\hline 153 & M4.75 & 2.85 & 1.84 & 4.76 & thick & $\mathrm{n}$ & PD & PD & PD \\
\hline 194 & M4.75 & 3.1 & 1.46 & 4.88 & thin & $\mathrm{n}$ & PD & PD & $\mathrm{TD}(\mathrm{HD})$ \\
\hline 213 & M4.75 & 1.4 & 1.48 & 4.04 & thin & $\mathrm{n}$ & PD & PD & $\mathrm{TD}(\mathrm{HD})$ \\
\hline 214 & M4.75 & 1.1 & 1.48 & 4.69 & thin & $\mathrm{n}$ & PD & PD & TD(HD) \\
\hline 301 & M4.75 & 6.2 & 1.24 & 4.93 & thin & $\mathrm{y}$ & TD & TD & $\mathrm{TD}(\mathrm{IH})$ \\
\hline 308 & M4 & 11.8 & 1.31 & 4.92 & thin & y & PD & TD & $\mathrm{TD}(\mathrm{IH})$ \\
\hline 1928 & M5.5 & 1.4 & 1.74 & 3.68 & thick & $\mathrm{n}$ & PD & PD & PD \\
\hline 8078 & M0.5 & 5.8 & 1.82 & 5.22 & thick & $\mathrm{n}$ & PD & PD & PD \\
\hline 9024 & M0 & 3.75 & 1.94 & 4.72 & thick & $\mathrm{n}$ & PD & PD & PD \\
\hline 10352 & M1 & 3.5 & 1.88 & 5.24 & thick & $\mathrm{n}$ & PD & PD & PD \\
\hline
\end{tabular}

Notes. $A_{V}$ (Best) corresponds to the extinction that produces the best fit to the observed SED. The range in $A_{V}$ used for SED modeling with the Robitaille models follows the method described for Taurus stars. The identification "Lu10" refers to Luhman et al. (2010), where the classification listed is based on applying the Luhman et al. (2010) color criteria. The classification listed for CK09, Currie \& Kenyon (2009), is based on applying their color criteria. The disk states listed here are considered to be "provisional" since IC 348 lacks sensitive far-IR and submillimeter data. 
Table 9

Provisional Disk Evolutionary States for Late-Type NGC 2362 Members Based on 1-24 $\mu$ m Data

\begin{tabular}{|c|c|c|c|c|c|c|c|c|c|}
\hline ID & ST & $A_{v}$ (Best) & $K_{s}-[8]$ & $K_{s}-[24]$ & $\begin{array}{c}\text { Disk State Classification } \\
\tau_{\text {mid-IR }} \\
\end{array}$ & Inner Hole? $\left(R_{\text {sub }}, \mathrm{AU}\right)$ & CL09 & Lu10 & This Work \\
\hline 3 & $\mathrm{M}^{\mathrm{a}}$ & 0.6 & 1.45 & 5.89 & Thin & $\mathrm{y}, 4.10$ & $\mathrm{TD}(\mathrm{IH})$ & $\mathrm{PD}$ & $\mathrm{TD}(\mathrm{IH})$ \\
\hline 36 & $\mathrm{~K} 5^{\mathrm{a}}$ & 0.5 & 1.70 & 4.51 & Thin & $\mathrm{n}$ & $\mathrm{TD}(\mathrm{HD})$ & $\mathrm{PD}$ & $\mathrm{TD}(\mathrm{HD})$ \\
\hline $41^{\mathrm{b}}$ & $\mathrm{K} 1$ & 0.3 & 0.19 & 1.34 & Thin & $\mathrm{n}$ & $\mathrm{TD}(\mathrm{HD})$ & $\ldots$ & $\mathrm{TD}(\mathrm{HD})$ \\
\hline $63^{b}$ & $\mathrm{M} 0^{\mathrm{a}}$ & 0.7 & 0.52 & 2.76 & Thin & $\mathrm{n}$ & $\mathrm{TD}(\mathrm{HD})$ & $\cdots$ & $\mathrm{TD}(\mathrm{HD})$ \\
\hline 85 & $\mathrm{M} 2^{\mathrm{a}}$ & 1.5 & 1.55 & 4.58 & Thin & $\mathrm{n}$ & $\mathrm{TD}(\mathrm{HD})$ & PD & $\mathrm{TD}(\mathrm{HD})$ \\
\hline 111 & $\mathrm{~K} 7$ & 0.75 & 2.5 & 5.34 & Thick & $\mathrm{n}$ & PD & PD & PD \\
\hline 139 & M2 & 0.4 & 3.48 & 6.82 & Thick & $\mathrm{n}$ & PD & PD & PD \\
\hline 168 & K3 & 0.5 & 0 & 3.48 & Thin & $\mathrm{y}, 5.20$ & $\mathrm{TD}(\mathrm{IH})$ & TD & $\mathrm{TD}(\mathrm{IH})$ \\
\hline 177 & $\mathrm{M} 0^{\mathrm{a}}$ & 0.8 & 1.82 & 5.33 & Thin & $?^{\mathrm{c}}$ & $\mathrm{TD}(\mathrm{HD})$ & PD & $\mathrm{PD} / \mathrm{TD}(\mathrm{HD}) / \mathrm{TD}(\mathrm{IH}) ?$ \\
\hline 187 & M0.5 & 1.5 & 2.64 & 4.81 & Thick & $\mathrm{n}$ & PD & PD & PD \\
\hline 194 & K5 & 1.2 & 1.06 & 5.46 & Thin & $\mathrm{y}, 7.57$ & $\mathrm{TD}(\mathrm{IH})$ & TD & $\mathrm{TD}(\mathrm{IH})$ \\
\hline 196 & $\mathrm{~K} 5^{\mathrm{a}}$ & 0.1 & 0.22 & 2.35 & Thin & $\mathrm{y}, 3.63$ & $\mathrm{TD}(\mathrm{IH})$ & $\mathrm{TD}$ & $\mathrm{TD}(\mathrm{IH})$ \\
\hline 202 & M2.5 & 0.9 & 2.53 & 5.92 & Thick & $\mathrm{n}$ & PD & PD & PD \\
\hline 204 & M2.5 & 2 & 1.46 & 4.69 & Thin & $\mathrm{n}$ & $\mathrm{TD}(\mathrm{HD})$ & PD & $\mathrm{TD}(\mathrm{HD})$ \\
\hline 213 & $\mathrm{M}^{\mathrm{a}}$ & 0.8 & 1.28 & 3.84 & Thin & $\mathrm{n}$ & $\mathrm{TD}(\mathrm{HD})$ & $\mathrm{TD}$ & $\mathrm{TD}(\mathrm{HD})$ \\
\hline 214 & $\mathrm{~K} 7^{\mathrm{a}}$ & 0.35 & 1.5 & 4.17 & Thin & $\mathrm{n}$ & $\mathrm{TD}(\mathrm{HD})$ & $\mathrm{PD}$ & $\mathrm{TD}(\mathrm{HD})$ \\
\hline 219 & M0 & 0.8 & 1.65 & 3.87 & Thin & $\mathrm{n}$ & $\mathrm{TD}(\mathrm{HD})$ & PD & $\mathrm{TD}(\mathrm{HD})$ \\
\hline 229 & M1.5 & 0.95 & 1.38 & 3.92 & Thin & $\mathrm{n}$ & $\mathrm{TD}(\mathrm{HD})$ & PD & $\mathrm{TD}(\mathrm{HD})$ \\
\hline 251 & $\mathrm{M} 2^{\mathrm{a}}$ & 1.25 & 1.7 & 4.68 & Thin & $\mathrm{n}$ & $\mathrm{TD}(\mathrm{HD})$ & PD & $\mathrm{TD}(\mathrm{HD})$ \\
\hline 267 & $\mathrm{M} 2^{\mathrm{a}}$ & 0.85 & 1.33 & 4.19 & Thin & $\mathrm{n}$ & $\mathrm{TD}(\mathrm{HD})$ & PD & $\mathrm{TD}(\mathrm{HD})$ \\
\hline 295 & $\mathrm{M} 2^{\mathrm{a}}$ & 0.4 & 0.34 & 4.20 & Thin & $\mathrm{y}, 1.29$ & $\mathrm{TD}(\mathrm{IH})$ & TD & $\mathrm{TD}(\mathrm{IH})$ \\
\hline
\end{tabular}

Notes. The identification "Lu10" refers to Luhman et al. (2010), where the classification listed is based on applying the Luhman et al. (2010) color criteria. The identification CL09 refers to the disk evolutionary states listed in Currie et al. (2009). The disk states listed from this work are considered to be provisional since NGC 2362 lacks sensitive far-IR/submillimeter data. To compare our results with those presented in Currie et al. (2009), we list the inner hole sizes in AU.

${ }^{a}$ Denotes approximate spectral types assigned based on fitting the optical and near-IR data. Likewise, $A_{V}$ (best) corresponds to the extinction that produces the best fit to the observed SED.

$\mathrm{b}$ The detections of these sources were confirmed by a rereduction of the MIPS $24 \mu \mathrm{m}$ data and PRF-fitting photometry.

c The best-fit models are evenly divided between those that have an AU-scale inner hole and those that do not. For members with disks that have an inner hole (marked "y"), we add the inner hole size in AU as determined in Currie et al. (2009).

for Taurus in this work shows that many sources with weak/ negligible $1-8 \mu \mathrm{m}$ emission and optically thick $24 \mu \mathrm{m}$ emission require inner holes while many with marginally optically thin near-to-mid-IR emission have low inferred disk masses. Figure 15 displays four representative SEDs comparing the observed emission to the optically thick, flat disk limit.

Our results are consistent with those from Currie et al. (2009) who argue that transitional disks are equal to or greater in number than primordial disks. Currie et al. (2009) find that $81 \%$ of disks around $\mathrm{K} 0-\mathrm{M} 3$ members are transitional disks $(17 / 21)$. Here four disks are primordial disks (IDs 111, 139, 187, and 202), and one (ID 177) appears to be a borderline case (since it may have an inner hole) but is counted as a primordial disk in the analysis below to be conservative (since it has $\left.K_{s}-[8]>1.75\right)$. The rest $(16 ; 14$ of which are K5-M3 stars) have near-to-mid-IR emission consistent with being transitional disks. Of these, five K5-M3 stars either have $K_{s}-[8] \leqslant 1.25$ (thus lying outside the range of colors contaminated by primordial disks) or show evidence for inner holes. The other nine K5-M3 stars have mid-IR colors between $K_{s}-[8]=1.25$ and 1.75 , within the contaminated region. Based on our Taurus analysis, two K5-M2 stars and 0-1 M2.5-M3 stars provisionally identified as having transitional disks instead have primordial disks with dust settling. Therefore, we arrive at a final transitional disk frequency of 58\%-63\% (11/19-12/ 19) for K5-M3 stars. The frequency for K5-M2 stars (63\%) and M2.5-M3 stars (33\%-66\%) is statistically indistinguishable given the small number in the latter group (3).

\subsection{Frequency of Transitional Disks in $\eta$ Cha}

Table 10 summarizes our modeling results for $\eta \mathrm{Cha}$. Of the 15 late-type members with IRAC and MIPS photometry, eight have evidence for a disk. Only three sources (RECX-11, J0843, and J0844) fulfill our definition of a primordial disk. Three members have transitional disks with inner holes (RECX-3, 4, and 5); two have homologously depleted transitional disks (RECX-9 and J0841; Figure 16). Thus, $\eta$ Cha has a high transitional disk frequency $(5 / 8$ or $63 \%)$. Our results agree with those of Sicilia-Aguilar et al. (2009) who separately analyze Spitzer IRS data to find that transitional disks comprise $50 \%-75 \%$ of the disk population. A "probabilistic" estimate of the transitional disk fraction (e.g., as in Section 4.3) yields the same answer: $n(\mathrm{TD})=4.95$ or $\sim 5$.

Gas diagnostics are consistent with our argument that most disks in $\eta$ Cha are transitional disks. RECX-5 and 9 are accreting at extremely low rates $\left(4-5 \times 10^{-11} M_{\odot} \mathrm{yr}^{-1}\right)$ (Lawson et al. 2004). RECX-3, RECX-4, and J0841 lack evidence for accretion circumstellar gas. RECX-3, 5, and 9 lack evidence for rovibrational $\mathrm{H}_{2}$ emission from warm circumstellar gas (Ramsay-Howat \& Greaves 2007). Only J0843, identified as a primordial disk from SED modeling, shows evidence for $\mathrm{H}_{2}$ emission and for substantial accretion $\left(10^{-9} M_{\odot} \mathrm{yr}^{-1}\right)$.

\subsection{Revised Transitional Disk Frequency for the Coronet Cluster}

Based on our analysis of Taurus data in Section 4, some fraction of Coronet Cluster stars determined to have transitional 

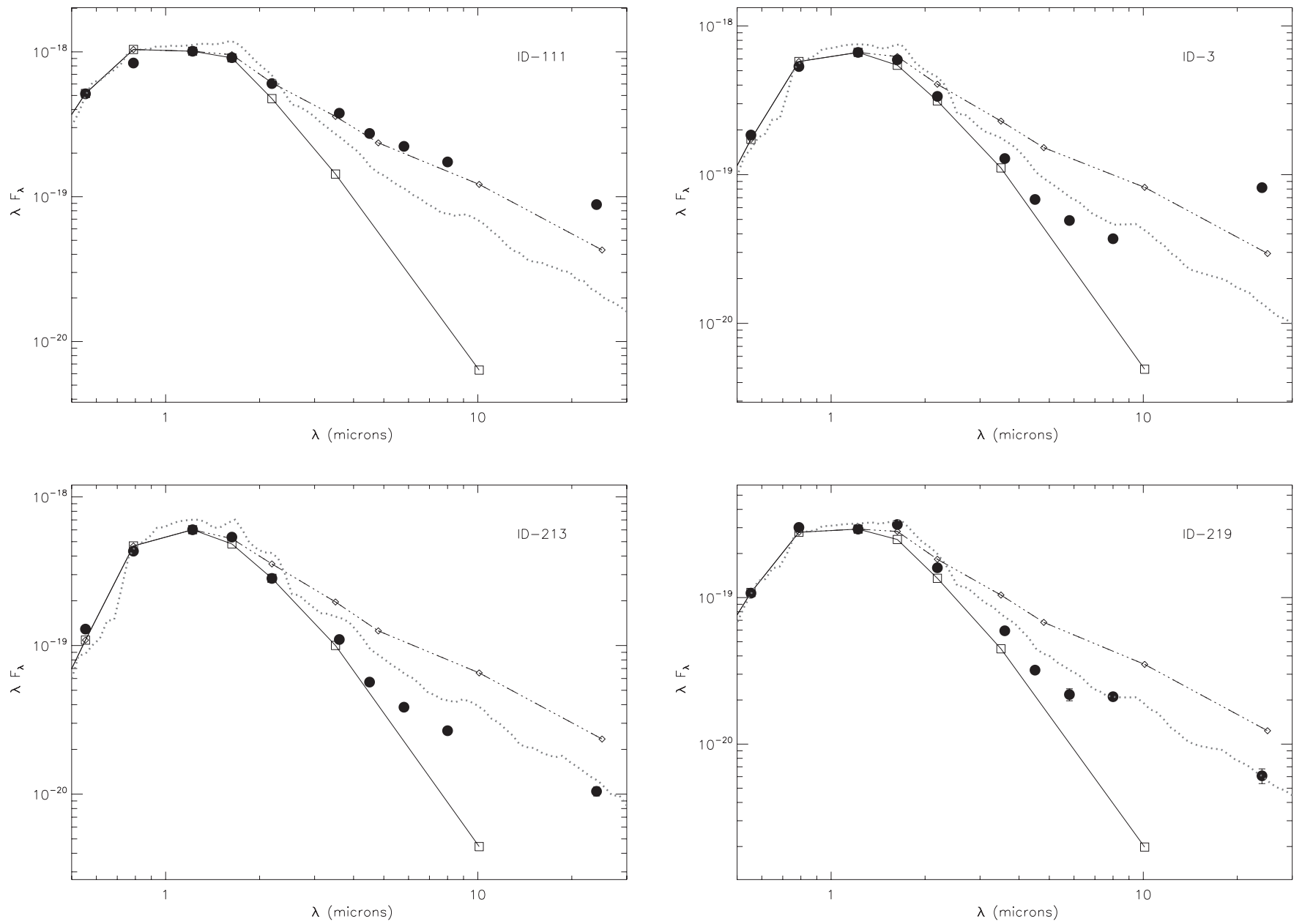

Figure 15. Representative SEDs for NGC 2362 sources. The provisional disk states for these members are (clockwise from top left): primordial disk, transitional disk with inner hole, homologously depleted transitional disk, and homologously depleted transitional disk.

Table 10

Disk Evolutionary States for Late-Type $\eta$ Cha Sources

\begin{tabular}{|c|c|c|c|c|c|c|c|c|c|c|c|c|}
\hline Name & $\begin{array}{l}\mathrm{ST}^{\mathrm{a}} \\
\left(M_{\odot}\right)\end{array}$ & $M_{\star}$ & $K_{s}-[8]$ & $K_{s}-[24]$ & $\tau_{\text {mid-IR }}$ & Inner Hole? & $M_{\text {disk }}$ & $\begin{array}{c}\text { Disk Classification } \\
\frac{M_{\text {disk }}}{M_{\star}}\end{array}$ & $f\left(<1.3 \times 10^{-3}\right)$ & $\mathrm{Si09}$ & Lu10 & This Work \\
\hline RECX-1 & $\mathrm{K} 7+\mathrm{M} 0, \mathrm{~K} 6$ & 0.95 & 0.23 & 0.38 & & $\mathrm{n}$ & & $\ldots$ & . & Star & Star & Star \\
\hline RECX-3 & M3, M3.25 & 0.35 & 0.26 & 0.92 & Thin & $\mathrm{y}$ & $3.5 \times 10^{-8}$ & $1 \times 10^{-7}$ & 1,1 & $\mathrm{TD}(\mathrm{IH})$ & TD & $\mathrm{TD}(\mathrm{IH})$ \\
\hline RECX-4 & M1.75, M1.3 & 0.6 & 0.30 & 0.89 & Thin & $\mathrm{y}$ & $1.8 \times 10^{-7}$ & $3 \times 10^{-7}$ & 1,1 & $\mathrm{TD}(\mathrm{IH})$ & $\mathrm{TD}$ & $\mathrm{TD}(\mathrm{IH})$ \\
\hline RECX-5 & M3.8, M4 & 0.25 & 0.97 & 4.81 & Thin & $\mathrm{y}$ & $7 \times 10^{-4}$ & $2.8 \times 10^{-3}$ & $0.14,0.73$ & $\mathrm{TD}(\mathrm{IH})$ & TD & $\mathrm{TD}(\mathrm{IH})$ \\
\hline RECX-6 & M3, M3 & 0.375 & 0.25 & 0.41 & $\ldots$ & $\mathrm{n}$ & $\ldots$ & $\ldots$ & $\ldots$ & Star & Star & Star \\
\hline RECX-7 & K6.9+M1, K6 & 0.95 & 0.17 & 0.26 & $\ldots$ & $\mathrm{n}$ & $\ldots$ & $\ldots$ & $\ldots$ & Star & Star & Star \\
\hline RECX-9 & M4.4+M4.7, M4.5 & 0.225 & 1.37 & 3.96 & Thin & $\mathrm{n}$ & $1.4 \times 10^{-4}$ & $6 \times 10^{-4}$ & $0.53,0.75$ & $\mathrm{TD}(\mathrm{HD})$ & PD & $\mathrm{TD}(\mathrm{HD})$ \\
\hline RECX-10 & M0.3, M1 & 0.6 & 0.24 & 0.28 & $\ldots$ & $\mathrm{n}$ & $\ldots$ & $\ldots$ & $\ldots$ & Star & Star & Star \\
\hline RECX-11 & K6.5, K5.5 & 1 & 1.69 & 3.98 & Thin & $\mathrm{n}$ & $3.3 \times 10^{-3}$ & $3.3 \times 10^{-3}$ & $0.13,0.50$ & PD & PD & $\mathrm{PD}$ \\
\hline RECX-12 & M3.2, M3.25 & 0.35 & 0.34 & 0.46 & $\ldots$ & $\mathrm{n}$ & $\ldots$ & $\ldots$ & $\ldots$ & Star & Star & Star \\
\hline J0841 & M5.5, M4.75 & 0.2 & 1.50 & 3.91 & Thin & $\mathrm{n}$ & $3 \times 10^{-5}$ & $2 \times 10^{-4}$ & 1,1 & $\mathrm{TD}(\mathrm{HD})$ & $\mathrm{PD}$ & $\mathrm{TD}(\mathrm{HD})$ \\
\hline J0843 & M5, M3.25 & 0.35 & 2.92 & 5.91 & Thick & $\mathrm{n}$ & $1 \times 10^{-3}$ & $3 \times 10^{-3}$ & $0.31,0.50$ & PD & PD & PD \\
\hline J0844 & M5.3, M5.75 & 0.1 & 1.86 & 4.51 & Thin? & $\mathrm{n}$ & $\ldots$ & $\ldots$ & $\ldots$ & PD & $?$ & $\mathrm{PD} / \mathrm{TD}(\mathrm{HD})^{\mathrm{b}}$ \\
\hline J0838 & M5, M5.25 & 0.15 & 0.53 & $\ldots$ & $\ldots$ & $\mathrm{n}$ & $\ldots$ & $\ldots$ & $\ldots$ & Star & Star & Star \\
\hline J0836 & M5.3, M5.5 & 0.12 & 0.58 & $\ldots$ & $\ldots$ & $\mathrm{n}$ & $\ldots$ & $\ldots$ & $\ldots$ & Star & Star & Star \\
\hline
\end{tabular}

Notes. The identification "Lu10" refers to Luhman et al. (2010), where the classification listed is based on applying the Luhman et al. (2010) color criteria. The identification "Si09" refers to Sicilia-Aguilar et al.'s (2009) disk classification translated into our nomenclature.

${ }^{a}$ The first entry for spectral type comes from Lyo et al. (2004) and the second one comes from Luhman \& Steeghs (2004). We compute the stellar masses and fractional disk masses using the Luhman \& Steeghs (2004) spectral types and the stellar masses for a given effective temperature at 6 Myr from the Baraffe (1998) isochrones. Our results do not leverage on whose spectral types we adopt.

${ }^{\mathrm{b}}$ For our statistics, we classify this disk as a primordial disk since it lacks far-IR data to needed to derive a meaningful disk mass estimate. We omit a column for optical extinction since it is negligible for this cluster (e.g., Lyo et al. 2004; Luhman \& Steeghs 2004). 

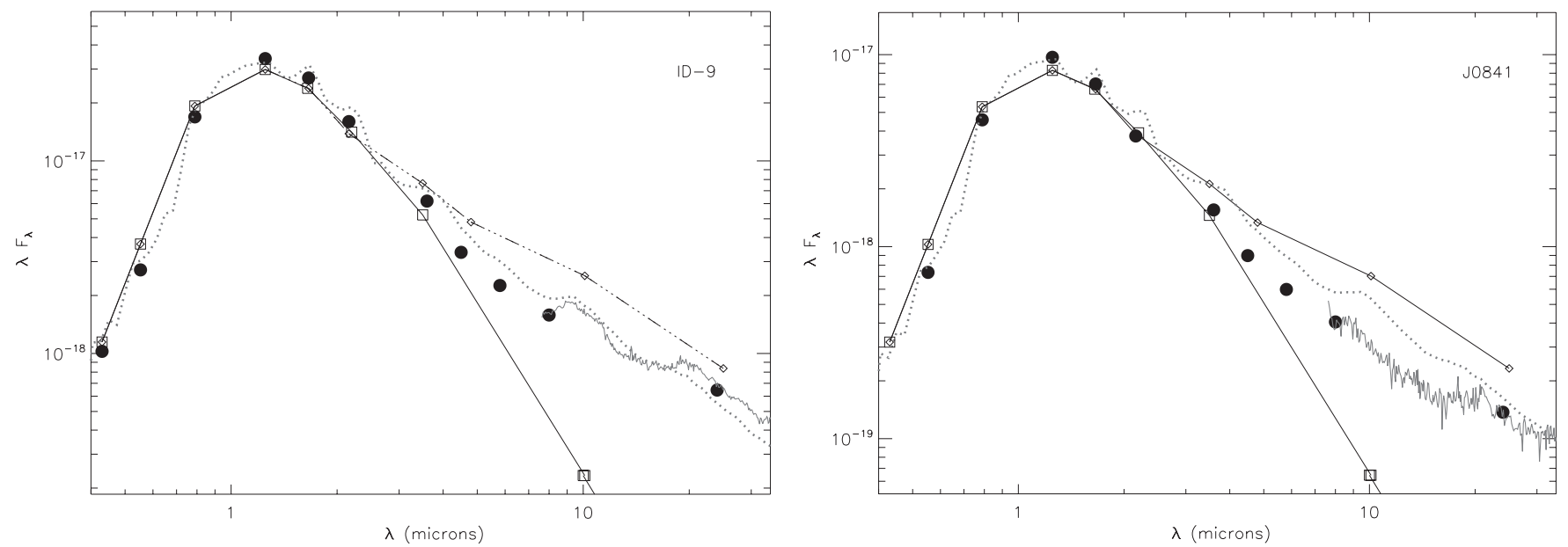

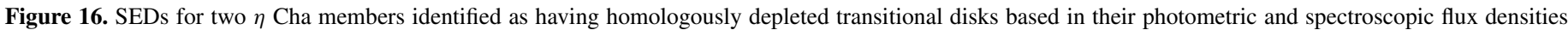
(dots and thick gray line, respectively) compared to the appropriate flat, reprocessing disk models.

Table 11

Frequency of Transitional Disks With Time (Corrected for Contamination)

\begin{tabular}{lccll}
\hline \hline Cluster & Age $(\mathrm{Myr})$ & Spectral-Type Range & \multicolumn{1}{c}{$f(\mathrm{TD})_{\text {lower }}$} & \multicolumn{1}{c}{$f(\mathrm{TD})_{\text {upper }}$} \\
\hline Taurus & $1-2$ & K5-M6 & $0.18(25 / 143)$ & $0.22(32 / 145)$ \\
& & K5-M2 & $0.17(13 / 75)$ & $0.21(16 / 77)$ \\
& & M2.5-M6 & $0.18(12 / 68)$ & $0.24(16 / 68)$ \\
Coronet & \multirow{2}{*}{$1-3$} & K5-M6 & $0.21(4 / 19)$ & $0.36(8 / 22)$ \\
& & K5-M2 & $0.12(1 / 9)$ & $0.22(2 / 9)$ \\
IC 348 & \multirow{2}{*}{2.5} & M2.5-M6 & $0.30(3 / 10)$ & $0.50(6 / 12)$ \\
& & K5-M6 & $0.32(40 / 124)$ & $0.40(49 / 124)$ \\
& & K5-M2 & $0.18(6 / 33)$ & $0.24(8 / 33)$ \\
NGC 2362 & 5 & M2.5-M6 & $0.42(34 / 81)$ & $0.51(41 / 81)$ \\
$\eta$ Cha & $6-8$ & K5-M3 & $0.58(11 / 19)$ & $0.63(12 / 19)$ \\
& & K5-M6 & $0.63(5 / 8)$ &
\end{tabular}

Notes. The transitional disk frequency is defined as $f(\mathrm{TD}) /(f(\mathrm{TD})+f(\mathrm{PD}))$. The range in transitional disk frequencies $(f(\mathrm{TD}))$ for Taurus accounts for disks with uncertain/indeterminable states. The range in frequencies for the Coronet, IC 348, and NGC 2362 also accounts for the range of contamination estimates based on SED modeling of Taurus data.

disks based on mid-IR data probably are primordial disks with dust settling. In particular, G-1 (M0), G-14 (M4.5), and G-87 (M1.5) have $K_{s}-[8]$ colors placing them within the contaminated region: other transitional disks have inner holes or lie within the uncontaminated region. Accounting for contamination, the size of the transitional disk population drops to $11 \%-22 \%$ for K5-M2 stars $(1 / 9,2 / 9)$ and 30\%-50\% for M2.5-M6 stars (3/ $10,6 / 12)$, where the ranges account both for contamination by primordial disks with substantial dust settling and for possible misclassification of debris disk candidates. Thus, our transitional disk frequencies for all five clusters now account for uncertainties in disk classification based on mid-IR data alone.

\subsection{The Evolution of the Transitional Disk Population}

Combining our results for the Coronet Cluster, Taurus, IC 348, NGC 2362, and $\eta$ Cha, we now investigate the frequency of transitional disks as a function of age and stellar properties. Table 11 lists the relative frequency of transitional disks as a function of time in three spectral-type bins: mid-K to mid-M stars (K5-M6), K5-M2 stars, and M3-M6 stars. To compare with theoretical predictions, we overplot the approximate loci (Figure 17, top panel, solid and dotted lines) expected from the Alexander \& Armitage (2009) disk evolution/planet formation models, which yield frequencies for transitional disks as a function of time and assume a disk clearing timescale of $\sim 0.5 \mathrm{Myr}$.

The transitional disk frequency for K5-M6 stars increases as a function of time from $\sim 20 \%$ at $1-2 \mathrm{Myr}$ to $\sim 50 \%-60 \%$ by $5 \mathrm{Myr}$. The frequencies are consistently 1.5-3 times higher than those predicted by Alexander \& Armitage (2009) for a $0.5 \mathrm{Myr}$ transition timescale. Within the context of the Alexander \& Armitage (2009) study, our results indicate that the transitional disk phase on average lasts longer than $0.5 \mathrm{Myr}$.

The bottom panel of Figure 17 compares our frequencies with those from Muzerolle et al. (2010) who use a more empirically based approach to identify transitional disks based primarily on IRAC and MIPS flux slopes (bottom panel). Our transitional disks frequencies are substantially larger than those Muzerolle et al. derive based on their self-described classical definition for what constitutes a transitional disk (diamonds): a disk with an inner hole and optically thick outer disk. However, Muzerolle et al. argues for an expanded definition for a transitional disk, including disks whose mid-IR emission plausibly identifies a more homologous depletion of emitting dust and disks with inner holes/cavities and more optically thin outer disks. Adopting this definition, their transitional disk frequencies (triangles) are in good agreement with those we derive for clusters older than 2 Myr. For the two clusters we both analyze, IC 348 and $\eta$ Cha, our frequencies are nearly identical.

To derive a timescale for the transitional disk phase, we follow a parametric, Monte Carlo approach, similar to that from Muzerolle et al. (2010), evolving a population of $10^{5}$ stars to simulate the frequency of disks in different states for clusters with ages less than 10 Myr. We adopt a 3 Myr $e$ folding timescale for the protoplanetary disk stage, consistent with Spitzer observations (Figure 18, top panel). Some fraction of the protoplanetary disk lifetime is spent in the transitional disk phase. To assess the duration of this phase, we vary the transitional disk $e$-folding timescale $\left(t_{\mathrm{TD}}\right)$; correspondingly, the $e$-folding timescale for the primordial disk phase is $3 \mathrm{Myr}-t_{\mathrm{TD}}$. Through the first $0.54 \mathrm{Myr}$, we fix the disk fraction at 1 to account for the lifetime of Class I protostars (Evans et al. 2009). Finally, we assume a $1 \mathrm{Myr}$ age dispersion at each cluster age.

As shown by Figure 18 (bottom panel), transitional disk frequencies in all clusters are most consistent with a $1 \mathrm{Myr}$ transition timescale, lying well above predictions for a $0.1-0.5 \mathrm{Myr}$ 



Figure 17. Frequency of transitional disks vs. time. Top panel: we compare our frequencies to the theoretical models of Alexander \& Armitage (2009) who determine the frequency of transitional disks vs. time from a disk evolution/planet formation model with a disk clearing timescale of $0.5 \mathrm{Myr}$. The dashed line corresponds to the reference model, while the solid line includes a dispersion in disk properties (initial angular momentum, disk lifetime, disk mass). See Alexander \& Armitage (2009) for details. Bottom panel: our frequencies compared to those derived by Muzerolle et al. (2010). Their loci correspond to frequencies assuming that all transitional disks have inner holes and optically thick outer regions (lower dashed line/diamonds) and allowing for a broader definition of transitional disk that would include many identified from this work (upper dashed line/triangles). In this figure and the next one, the dots identify the average of the lower and upper limits for the transitional disk frequency as listed in Table 11.

timescale and below predictions for a $2 \mathrm{Myr}$ timescale. Thus, our analysis confirms earlier results based on NGC 2362 and $\eta$ Cha from Currie et al. (2009) and Sicilia-Aguilar et al. (2009) that the mean transitional disk lifetime must be an appreciable fraction of the total protoplanetary disk lifetime, estimated to be 3-5 Myr (Hernandez et al. 2007a; Currie et al. 2009). Adopting Muzerolle et al.'s (2010) model for the frequency of transitional disks with time yields the same qualitative result. They predict smaller transitional disk frequencies at 3-10 Myr than we do for a $1 \mathrm{Myr}$ transition timescale. The identification of a long transition timescale based on our predictions is then conservative. On the other hand, our results are in conflict with other recent claims, particularly those of Luhman et al. (2010) who argue for a transitional disk lifetime less than 0.5 Myr. In
Section 5, we compare our methodology with theirs and those from other recent studies.

We emphasize that our disk frequencies account for disks with both transitional disk morphologies: disks with inner holes and homologously depleted disks. Homologously depleted transitional disks generally appear to be more numerous than those with inner holes, up to a factor of 1.5-2 larger in number for the Coronet Cluster and NGC 2362. However, these differing frequencies do not constrain the relative lifetimes of disks following these evolutionary paths: we cannot tell between a morphology with a shorter lifetime and one that operates less frequently. We also note that these results are statistical. In other words, while there is some uncertainty in the state for individual objects, these uncertainties are averaged out at the end. 



Figure 18. Top: the frequency of protoplanetary disks vs. time as probed by Spitzer observations. For NGC 1333, IC 348 , and 25 Ori the frequencies are taken from the literature (Gutermuth et al. 2008; Lada et al. 2006; Hernandez et al. 2007b). The frequency for Taurus is taken from the number of "Class II" objects in Taurus reported by Luhman et al. (2010) and the number of "Class III" objects showing evidence for weak IR excess (e.g., FW Tau). The frequency for NGC 2362 is taken from data presented in Currie et al. (2009), which is in agreement with estimates from Dahm \& Hillenbrand (2007). Bottom: the transitional disk frequency vs. time for different clusters in our study. Overplotted are the predicted transitional disk frequencies from our parametric model, assuming a typical protoplanetary disk lifetime of $3 \mathrm{Myr}$ and varying amounts of that time spent as transitional disks (0.1-2 Myr).

At least for the Coronet Cluster and IC 348, the transitional disk frequency is spectral type dependent (Figure 19). The transitional disk frequencies for M3-M6 stars in these clusters is marginally but consistently higher than those for K5-M2 stars by about a factor of $\sim 1.5$. Formally, M3-M6 stars in Taurus also more frequently have transitional disks than do K5-M2 stars, though the difference is not statistically significant. Muzerolle et al. (2010) also find that the transitional disk population is dominated by later-type stars, though their correlation is stronger than what we find.

A spectral-type-dependent transitional disk frequency may help explain the high transitional disk frequency found by Sicilia-Aguilar et al. (2008). Since the Sicilia-Aguilar et al. (2008) sample is drawn heavily from stars later than M2, their sample is predisposed toward having a high transitional disk fraction compared to cluster-averaged values and compared to those for brighter, more easily detectable $\mathrm{K}$ to $\mathrm{M} 2$ stars. The transitional disk frequency for the bulk cluster population (e.g., including members in Table 3) is similar to that for other 1-3 Myr old clusters. At least one other cluster, IC 348, also has a very high transitional disk fraction for very low mass stars.

\section{DISCUSSION}

\subsection{Summary of Results}

This paper expands upon previous Spitzer analysis of the Coronet Cluster disk population around very low mass stars performed by Sicilia-Aguilar et al. (2008), presenting new 




Figure 19. Transitional disk frequency vs. time for different clusters separated by spectral type: K5-M2 stars (lower locus) and later stars (upper locus). The error bars simply define the range in values from Table 11, taking into account disks with uncertain states.

photometry for other cluster members, including many with higher stellar masses. We use SED modeling and comparisons with simple disk models/empirical metrics to assess the Coronet Cluster disk population, focusing on identifying and characterizing candidate transitional disks. By analyzing the disk population for other 1-8 Myr old clusters, we investigate the utility of using mid-IR data to probe disk evolutionary states, determine how the frequencies of transitional disks change with time, and investigate how this lifetime may depend on stellar properties. Our study yields the following major results.

1. The Coronet Cluster contains a high frequency of transitional disks: $\sim 30 \%$ (formally, $21 \%-36 \%$ ) of the disk population around K5-M6 stars. Although the samples are small, our analysis hints at a spectral-type-dependent frequency for transitional disks, confirming earlier suggestions by Sicilia-Aguilar et al. (2008, 2009): they appear more frequently around the very low mass stars that were the focus of Sicilia-Aguilar et al. (2008). IC 348 exhibits the same spectral-type/stellar-mass-dependent transitional disk frequency.

2. Based on optical-to-submillimeter SED analysis of Taurus sources with high-quality photometry, we confirm that many disks lacking clear evidence for inner holes also have a low dust mass. Their mid-IR emission is due to a depletion of dust, not dust settling: these represent a more homologous depletion of disk material with time instead of an inside-out dispersal. Many transitional disks can only be identified from SED modeling as their mid-IR colors overlap with primordial disks. About 22\%-33\% (10\%-50\%) of disks around K5-M2 (M3-M6) stars identified as homologously depleted transitional disks based on optical-tomid-IR data alone may in fact be primordial disks with a heavy depletion of submicron-sized dust but a large total dust mass. Disks with very different morphologies can occupy the same region of color-color space: SED modeling is required to avoid misclassifying disks.

3. Combining analysis of the Coronet Cluster disk population with that for Taurus, IC 348, NGC 2362 and $\eta$ Cha shows that the relative frequency of transitional disks around K5-M6 stars increases from $\sim 20 \%$ at 1-2 Myr to 50-60\% by 5-8 Myr. Parametric modeling shows that this trend is implies a mean transitional disk lifetime near $1 \mathrm{Myr}$, not $\sim 0.1-0.5 \mathrm{Myr}$.

\subsection{Transitional Disk Identification in Other Recent Work: The Importance of SED Modeling}

Other recent studies of transitional disks provide evidence that the weak-IR-to-submillimeter dust emission from homologously depleted transitional disks is likely due to disk clearing, consistent with our results. Cieza et al. (2008) selected transitional disks as those with mid-IR emission weaker than the lower-quartile Taurus SED and low levels of accretion, including many that lack any evidence for inner holes. They found that disks with weak mid-IR emission have low submillimeterinferred disk masses. Cieza et al. (2008) interpret this trend as evidence in favor of UV photoevaporation models for disk clearing: photoevaporation should begin to clear the inner disk once the total disk mass (probed by submillimeter data) drops significantly. More generally, though, this trend implies that for some disks the mass of emitting dust drops simultaneously over a wide range of stellocentric distance, consistent with a homologous depletion of disk material.

Cieza et al. (2010) found that the disk mass and accretion rate for transitional disks, including those we would identify as homologously depleted, appear to be correlated (Cieza et al. 2010): disks with lower accretion rates or small upper limits have lower inferred disk masses. There is no clear reason why the accretion rate should drop because submicron-sized grains had grown; however, disks undergoing a homologous depletion of their total mass in gas and dust should have low inferred masses and a lower frequency and rate of accretion.

Other studies, particularly the C2D survey of transitional disks presented in Merin et al. (2010), conclusively show that SED modeling is needed to properly characterize disks. By modeling optical through submillimeter photometry and spectroscopy of a mid-IR selected sample of candidate transitional disks ("cold disks" in their terminology), they determine which 
disks have inner holes and which lack evidence for an inner hole and investigate typical mid-IR colors for both kinds of disks. Their analysis shows that the mid-IR colors of transitional disks overlap with primordial disks (e.g., their Figure 15). Computing the frequency of transitional disks using mid-IR colors is then error prone unless SED modeling of well-characterized sources is used to identify uncontaminated regions of color-color space and quantitatively assess contamination where transitional disks and primordial disks have the same colors. This finding is qualitatively consistent with our Taurus modeling results.

Luhman et al. (2010) compile data for numerous 1-10 Myr old clusters to uniformly identify transitional disks and quantify their population self-consistently. Their modeling follows a different approach than that followed here and in some previous work (e.g., Currie et al. 2009; Merin et al. 2010), as they select synthetic $K_{s}-[8]$ and $K_{s}-[24]$ colors produced from the D'Alessio et al. (2006) 1+1D disk code as fiducial colors separating primordial disks from transitional disks. The model they use has two grain populations: where the "big" grain population extends from submicron to millimeter sizes and is confined to the disk midplane, while the "small" grains lie above the midplane and are $\lesssim 1 \mu \mathrm{m}$ in size. It assumes a negligible accretion rate $\left(\dot{M}=10^{-10} M_{\odot} \mathrm{yr}^{-1}\right)$ and a "depletion factor," $\epsilon=0.001$, which removes $99.9 \%$ of the small dust grains. They find lower transitional disk frequencies and thus derive a shorter lifetime for the transitional disk phase. They claim that previous work (e.g., Currie et al. 2009; Sicilia-Aguilar et al. 2009) arguing in favor of a longer transitional disk lifetime improperly identifies sources with emission weaker than the median Taurus SED as transitional disks or conflates a lack of near-IR emission with evidence for disk clearing. ${ }^{9}$

We agree with Luhman et al. (2010) that some primordial disks can have very blue colors due to dust settling. However, they did not quantitatively consider the possibility that some transitional disks may (in some color-color diagrams) have redder colors than the bluest primordial disks. Based on a more detailed, multiwavelength analysis, we derive higher transitional disk frequencies than they do for nearly all clusters, especially for those older than Taurus. Our disagreement primarily arises because many disks that Luhman et al. (2010) identify as being optically thick primordial disks from their color criteria are consistent with having optically thin/marginally optically thin emitting dust in the mid-IR and low inferred disk masses. If their weak mid-IR emission were due only to the growth and settling of the smallest grains, their far-IR to submillimeter emission (and thus inferred disk masses) should be comparable to known primordial disks, which is not observed. Therefore, these disks are more consistent with being transitional disks.

There are other aspects of Luhman et al.'s analysis that weaken or undermine their conclusions. First, while Luhman et al.'s (2010) adopted model results from a sophisticated code, their conclusions are prone to large uncertainties, mostly because they use color comparisons from a single model to identify transitional disks, not full SED modeling. Within the context of their model, the Luhman et al. conclusions are extremely sensitive to assumed values for their free parameters, $\epsilon$ and $\dot{M}$, and thus highly selective. Assuming either that disks remove $99 \%$ of their small dust instead of $99.9 \%$ or have a small accretion rate of $\dot{M}=10^{-9} M_{\odot} \mathrm{yr}^{-1}$ revises their fiducial mid-

\footnotetext{
9 As can be seen from a simple inspection of the Currie et al. (2009) analysis, their disk classification was instead based on comparing SEDs to the

lower-quartile Taurus SED. This was clarified in later work (Currie \& Kenyon 2009).
}

IR colors redward by $\sim 0.5$ mag (e.g., $K_{s}-[8] \sim 1.75, K_{s}-[24] \sim$ 4.5 for K5-M2 stars). Using these colors to classify disks yields results similar to those presented here and in Currie et al. (2009) for 15 of the 16 model combinations of $\epsilon(0.001-1)$ and $M$ $\left(10^{-10}-10^{-7} M_{\odot}\right)$ studied in D'Alessio et al. (2006, e.g., their Figure 13).

Second, while $\epsilon$ may be a true free parameter, measured accretion rates for many sources are much higher than that from the disk model used by Luhman et al. (2010), rendering their adopted model inapplicable. For example, ID 85 in NGC 2362, identified as a transitional disk by Currie et al. (2009), is a likely accretor based on its $\mathrm{H}_{\alpha}$ emission. Using relations from Dahm (2008) and Herczeg \& Hillenbrand (2008), ${ }^{10}$ its accretion rate is $\sim(4-7) \times 10^{-9} M_{\odot} \mathrm{yr}^{-1}$, or $40-70$ times greater than what Luhman et al. assume. From comparing the SED of ID 85 to output from D'Alessio et al. disk models with realistic accretion rates, this source should be classified as a transitional disk, contrary to Luhman et al.'s results. Taurus-Auriga, Trumpler 37, and other clusters analyzed by Luhman et al. include many sources with weak mid-IR emission (transitional disks/borderline cases) whose measured accretion rates render Luhman et al.'s analysis inapplicable, such as ZZ Tau $\left(1.3 \times 10^{-9} M_{\odot} \mathrm{yr}^{-1}\right.$; White \& Ghez 2001 , see also SiciliaAguilar et al. 2010).

Third, the model that Luhman et al. (2010) adopt likely (1) has an extremely low disk mass which (2) is almost never optically thick and thus cannot distinguish optically thick primordial disks from other disks. As shown in Tables 2.2 and 2.4 of Espaillat (2009), where the D'Alessio et al. input parameters are detailed, the disk mass for models with parameters adopted by Luhman is $\sim(1.38-1.45) \times 10^{-4} M_{\odot}$ : an order-of-magnitude (or more) smaller than our primordial/transitional disk division for all but the latest-type stars and the median primordial disk mass (Andrews \& Williams 2005; see also the Appendix). Furthermore, Figure 2.6 of Espaillat (2009) shows that almost none of this disk is optically thick to its own radiation $\left(\tau_{R} \gg\right.$ $1)$, unlike our adopted model and other D'Alessio et al. models. Thus, a major reason why the Luhman's adopted model yields weak mid-IR emission is because of its low dust mass, not just because of dust settling. To better parse disks into different states, Luhman et al. should have instead adopted a model yielding an optically thick disk with a dust mass comparable to typical primordial disk masses (e.g., the fiducial model in D'Alessio et al. 2006 except with $\epsilon=0.001$ and the accretion rate set to a negligible value).

Finally, SED modeling and imaging of individual sources shows that the Luhman et al. color criteria fail to uniquely identify primordial/transitional disks. For example, UX Tau and $\mathrm{LkCa} 15$ are identified as primordial disks according to Luhman et al.'s color criteria as they are redder than the authors' primordial disk limit. But SED modeling and/or high-contrast imaging show that both have large gaps with optically thin dust/ no dust extending from $\approx 0.1$ AU to $\approx 50$ AU from the star (Thalmann et al. 2010; Mulders et al. 2010; Espaillat et al. 2007). SED modeling also shows that sources in NGC 2362 (e.g., ID 3), IC 348 (e.g., ID 97), and Trumpler 37 (e.g., ID 14 11) classified by Luhman et al. as primordial disks probably

\footnotetext{
${ }_{10}$ Here, we assume $E(B-V)=0.01(1 / 10$ th the cluster average listed by Moitinho et al. 2001), an age of $5 \mathrm{Myr}$, a stellar radius for an M2 star of this age from Baraffe (1998), and $R_{\text {in }}=5 R_{\star}$. The intrinsic uncertainty in the accretion rate is $<0.5$ dex (see Dahm 2008). According to SED modeling presented here, the reddening is probably higher, meaning that the intrinsic $H_{\alpha}$ luminosity is higher and the resulting accretion rate is also higher.
} 
have inner holes and thus are transitional disks (e.g., Currie et al. 2009; Sicilia-Aguilar et al. 2007; this work).

Even though we disagree with Luhman et al.'s conclusions for reasons described above, their study reinforces a major point made here-mid-IR colors of disks can be highly degenerate. The degeneracy highlighted from both of our studies shows that full SED modeling is needed to accurately assess the frequency of transitional disks and the duration of the transitional disk phase. While time intensive, this approach provides de facto more information about disk properties than can be provided by comparing source colors with synthetic colors resulting from a single disk model in a single color-color diagram.

\subsection{Transitional Disks in Young Clusters: Why the Paucity of Transitional Disks in Taurus Does Not Imply a Short Timescale for Disk Clearing}

Many pre-Spitzer studies have argued that the small number of transitional disks compared to optically thick primordial disks and diskless stars in young clusters like Taurus provides evidence in favor of a short $(\lesssim 0.1 \mathrm{Myr})$ transition disk phase due to rapid disk clearing (e.g., Skrutskie 1990; Simon \& Prato 1995; Wolk \& Walter 1996; and later work). Though transitional disks are infrequent compared to primordial disks and diskless stars in the youngest clusters (e.g., 20\% for Taurus), it does not follow that the transitional phase must be very short lived (e.g., $\lesssim 0.1 \mathrm{Myr}$ ). Here, we review why this is the case, describe how our work and other recent studies support this position, and consider recent work that revives older claims.

As argued by Currie et al. (2009), stars in Taurus are typically too young compared to the mean protoplanetary disk lifetime (3-5 Myr) for the majority of them to begin clearing. Thus, it is unsurprising that relatively few show evidence of active clearing. Our parametric modeling quantitatively supports this argument, showing that transitional disks do not comprise the majority of disks in the youngest clusters even for lengthy transitional disk phases (e.g., $t_{\mathrm{TD}}=1 \mathrm{Myr}$ ). Thus, the size of the transitional disk population at $\sim 1-2$ Myr does not, by itself, clarify how long protoplanetary disks show signs of active clearing over a typical lifetime of 3-5 Myr. To explain the large population of diskless stars in Taurus ( $\sim 30 \%$ of the total population), Currie et al. (2009) argue that stars with close binary companions lose their protoplanetary disks far more rapidly than stars without close binary companions (e.g., Ireland \& Kraus 2008).

Luhman et al. disagree with both of these claims. They argue that age cannot explain Taurus's paucity of transitional disks, since diskless stars in Taurus are more dispersed and are thus older than stars with disks. We agree with Luhman et al. (2010) that an age spread may also explain the large number of diskless sources in Taurus. However, this fact would simply confirm that older stars are less likely to have disks (e.g., Sicilia-Aguilar et al. 2006b; Hernandez et al. 2007a; Currie et al. 2007, 2009) and thus does not provide evidence in favor of rapid disk clearing.

Luhman et al. also argue that binarity cannot explain the large diskless population in Taurus. They note that the Taurus binary frequency declines with mass. They claim that if many Taurus disks are cleared by binaries then the disk fraction should be highest at low masses, which is not observed. However, the drop in binary frequency for low-mass stars is heavily weighted toward a drop in wide binary companions with separations of $\sim 200$ AU or greater, which are irrelevant here (Kraus et al. 2006, 2011). Moreover, many diskless stars in Taurus are tight binaries (Kraus et al. 2011). With tight binaries removed, the population of diskless stars shrinks significantly. Cieza et al. (2009) also find that disks in short-period binary systems are less frequent than those around wide-separation binaries or single stars (see also Bouwman et al. 2006).

In summary, either binarity or an age spread explains the smaller number of Taurus stars with transitional disks relative to primordial disks and diskless stars. Moreover, parametric modeling shows that the frequency of protoplanetary disks in the transitional disk phase is perfectly consistent with an extended transitional disk phase. Thus, Taurus data provides no evidence that the transitional disk phase must be rapid.

\subsection{Limitations and Uncertainties of Our Study and Future Work}

\subsubsection{Qualifications on Our Identification of Transitional Disks}

Our identification of some transitional disks (those labeled as homologously depleted) is predicated on (1) our assumptions about the range of primordial disk masses, (2) the reliability of the Robitaille models in estimating disk masses, and (3) the reliability of our assumed stellar masses. Current data show that the lower quartile of masses for optically thick primordial disks is $0.003 M_{\star}$ and nearly all primordial disks have masses greater than $0.001 M_{\star}$. Since not all Taurus members have been tagged by submillimeter observations, it is possible that future surveys will uncover more disks with optically thick near-tomid-IR emission but inferred masses that are systematically lower than the interquartile range/full range from current observations.

Additionally, as shown in Figure 11, the best-fit disk models for homologously depleted transitional disks have median values for absolute disk masses that yield fractional disk masses lying below our adopted primordial disk limit. However, some of the models for each disk (typically, 10\%-30\%) yield masses lying above $0.001 M_{\star}$. Thus, it is possible, albeit unlikely, that some of these disks are not low mass.

However, submillimeter data constrain the mass of emitting, optically thin dust to be less than these limits. Moreover, the bestfit models for some primordial disks and all borderline cases include those with masses below $0.001 M_{\star}$. Thus, uncertainties in determining disk masses do not necessarily bias our results in favor of finding a high frequency of transitional disks. Similarly, the classification of some sources with optically thin near-tomid-IR emission but large inferred disk masses (e.g., V836 Tau) as primordial disks is conservative. While these sources lack clear evidence for cleaned inner holes/gaps, their inner regions may be optically thin, and they may be undergoing the early stages of inner disk clearing.

More generally, the intrinsic sampling of the Robitaille et al. grid is non-uniform across all fitting parameters, which in principle could undermine our conclusions. As mentioned previously, disks without inner holes are more frequently represented than those without holes and massive disks $\left(M_{\text {disk }} \sim 0.01 M_{\odot}\right)$ are also better represented than lower-mass disks. Figure 3 of Robitaille et al. indicates the presence of "coupled" non-uniform sampling between parameters: there are many more models with an envelope accretion rate greater than $10^{-6} M_{\odot} \mathrm{yr}^{-1}$ and that these models predominantly have disk masses greater than $10^{-3} M_{\odot}$.

However, our disk mass estimates are not undermined by nonuniform sampling. The lower two panels of Robitaille et al.'s Figure 3 and our Figure 20 indicate that simply including farIR and submillimeter data eliminates vast swaths of parameter space and overcomes these sampling biases. The intrinsic 

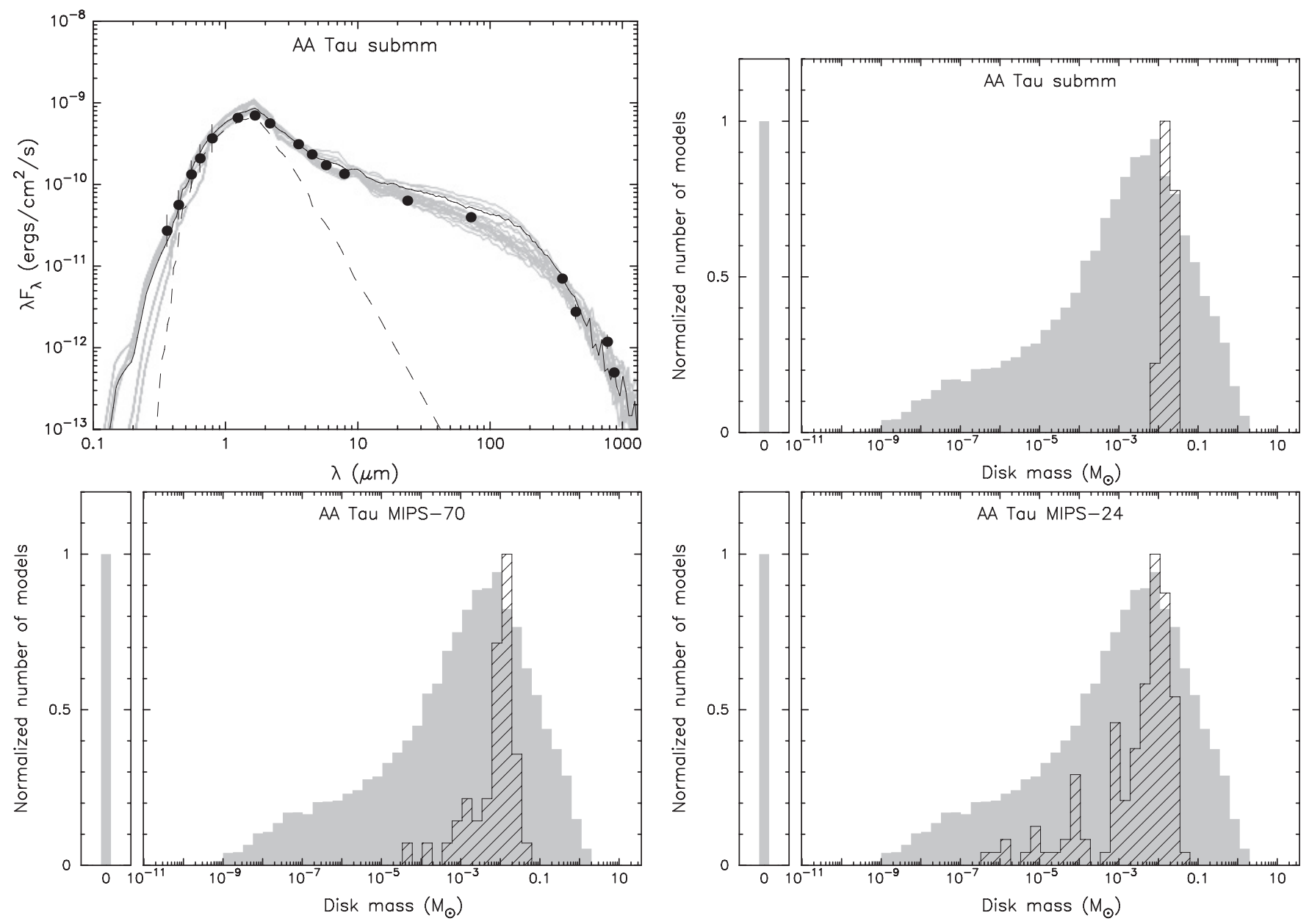

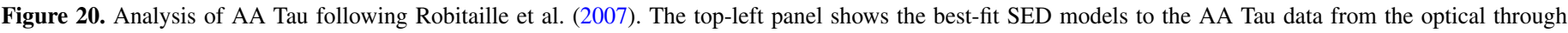

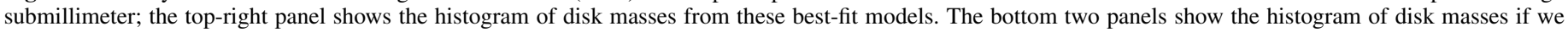
restricted our data to $70 \mu \mathrm{m}$ or less (bottom-left panel) and $24 \mu \mathrm{m}$ or less (bottom-right panel).

sampling of the Robitaille grid is weighted toward massive disks, yet we find numerous homologously depleted disks in spite of this bias because far-IR/submillimeter non-detections rule out nearly all massive disks. Conversely, the submillimeter detections for primordial disks provide strict limits on the inferred disk mass (see also Beckwith et al. 1990; Andrews $\&$ Williams 2005) and make the interquartile range of $M_{\text {disk }} /$ $M_{\star}$ for these objects nearly single valued in many cases (see Figure 13). On the other hand, uncertainties in deriving other disk parameters (e.g., flaring power) have not been fully explored here and may be seriously affected by non-uniform sampling.

Our analysis leverages not just on the precision with which we can estimate the mass of disks but also our accuracy. Estimating a mass of emitting dust from submillimeter data alone requires assuming a value for the dust opacity, $\kappa$ (e.g., Beckwith et al. 1990; Beckwith \& Sargent 1991; Henning \& Stognienko 1996; and later references). Since the dust opacity is not strictly known (and is affected by grain growth), what can be constrained from such data is the product of the dust mass and the opacity, not simply the dust mass. On the other hand, grain growth/dust settling (which affect $\kappa$ ) have far stronger effects on the near-to-mid-IR portion of disk SEDs than in the submillimeter (e.g., Wood et al. 2002; D'Alessio et al. 2006). For instance, "settled disk" models from D'Alessio et al. (2006) have 250-1000 $\mu \mathrm{m}$ emission equal to or larger than that for disks without grain growth/settling (see Figure 13, top two sets of panels in D'Alessio et al. 2006).

The total disk mass is also uncertain. Since continuum IR to submillimeter emission comes predominantly from dust, a gasto-dust ratio must be assumed in converting a submillimeterinferred dust mass to a total disk mass. The models used here, in Currie et al. (2009), and other work (e.g., Cieza et al. 2010; Andrews \& Williams 2005) assume standard values from $\kappa$ and a solar gas-to-dust ratio (see Beckwith \& Sargent 1991). However, comparisons with disk masses derived from accretion rates (assuming steady-state accretion) indicate that our methods may systematically underestimate true disk masses (e.g., Andrews \& Williams 2007). This may be due either to an inaccurate assumed value for $\kappa$ or a substantially non-solar gas-to-dust ratio, which may occur from grain growth and planetesimal formation. Our analysis provides evidence that masses for homologously depleted transitional disks are systematically lower by a factor of 10-1000 compared to primordial disks; other work provides evidence that gas and small dust deplete on similar timescales for disks in general and for homologously depleted transitional disks in particular (Fedele et al. 2010; Kennedy \& Kenyon 2009; Cieza et al. 2010). However, given the uncertainties in determining $\kappa$ and the gas-to-dust ratio, our analysis does not provide an absolute calibration for disk masses that can inform models for planet formation (e.g., Kenyon \& Bromley 2009; Bromley \& Kenyon 2011). 
Accurately quantifying uncertainties in stellar mass for each star is another challenge. Between $1 \mathrm{Myr}$ and $5 \mathrm{Myr}$, the Baraffe (1998) isochrones with a mixing length parameter of $L_{p}=1.9 H_{p}$ ("required to fit the Sun") predict that $1.4 M_{\odot}$ stars increase in $T_{\text {eff }}$ by $\sim 125 \mathrm{~K}$ from $4663 \mathrm{~K}$ to $4786 \mathrm{~K}$, thus changing in spectral type by $\sim 1$ subclass (cf. Currie et al. 2010). The variation is smaller $(\sim 100 \mathrm{~K})$ for solar-mass stars and inconsequential for very low mass stars $\left(M_{\star} \lesssim 0.7 M_{\odot}\right)$. Adopting the conversion from spectral type to mass from Baraffe, our stellar masses are then intrinsically uncertain by $\approx 0.1 M_{\odot}$ or less due to the different mapping between spectral type and mass as a function of age. The uncertainties in measured spectral types are up to 1-2 subclasses, considering the range of spectral types reported in the literature for various Taurus members (e.g., White \& Hillenbrand 2004). The age and spectral-type uncertainties listed above indicate that our fractional disk masses could be uncertain by an additional $10 \%-20 \%$, and that considering these uncertainties could slightly broaden the interquartile range of fractional disk masses for some objects.

Finally, our analysis may miss some sources whose gaps cannot be inferred from photometric data alone. Among our sample, the SED for DH Tau shows evidence for a $\lambda F_{\lambda} \propto \lambda^{-3}$ decline from $3.6 \mu \mathrm{m}$ to $8 \mu \mathrm{m}$, similar to that for transitional disks UX Tau and $\mathrm{LkCa} 15$, which have large (tens of $\mathrm{AU}$ ) gaps separating a small optically thick, $\sim 1500 \mathrm{~K}$ inner disk from an optically thick outer disk. The SED fit to this source from the Robitaille et al. grid is poor. Since the Robitaille et al. grid provides good sampling for full disks with a range of masses (and thus mid-IR emission) and disks with cleaned inner holes, it is plausible that DH Tau's disk has a different morphology such as a disk with a developing gap. Since not all Taurus members have been targeted by mid-IR spectrographs, more disks with small holes/gaps may be identified by future observations. Indeed, Espaillat et al. (2011) argue that IP Tau, which we identify as having a primordial disk in spite of its weak mid-IR emission, has a $\sim 2$ AU gap. Thus, the true size of the transitional disk population in Taurus, especially the subset with small gaps/inner holes, is plausibly larger than what we find.

\subsubsection{Limits on Our Study as a Probe of Transitional Disk Lifetimes/Disk Clearing}

While our analysis provides strong evidence in favor of a long transitional disk phase, especially for very low mass stars, several important caveats qualify this conclusion. First, many different mechanisms may be responsible for explaining the morphologies of transitional disks (Alexander \& Armitage 2009; Cieza et al. 2010; Sicilia-Aguilar et al. 2010). Therefore, a "transition timescale/transitional disk lifetime" derived from the relative frequency of all transitional disks is only an averaged value of the timescales from a number of different mechanisms like gas giant planet formation and UV photoevaporation. Some of these mechanisms may operate on fast, $\lesssim 0.1-0.5 \mathrm{Myr}$ timescales but less frequently whereas others take $\gtrsim 1 \mathrm{Myr}$ to clear dust in disks and operate more frequently.

The disk clearing time for many candidate mechanisms (e.g., gas giant planet formation and UV photoevaporation) depends on properties such as disk viscosity, initial disk mass, and initial angular momentum that are poorly constrained and likely have a large intrinsic dispersion. This dispersion in disk properties may produce a large dispersion in the duration of the transition phase similar to the observed dispersion in total protoplanetary disk lifetimes. While the mean transition timescale may be $\sim 1 \mathrm{Myr}$ as found here, in Currie et al. (2009), and Sicilia-Aguilar et al. (2009), the transitional disk phase for individual disks may last much shorter or longer.

Quantitatively assessing how transitional disks probe the clearing of both gas and dust requires diagnostics of circumstellar gas sensitive to the bulk gas content. The Herschel Space Observatory offers a sensitive probe of far-IR line emission to identify cool gas in planet-forming regions of the disk. Herschel programs such as GASPS will survey many 1-10 Myr old stars for evidence of circumstellar gas and thus provide strong constraints on gas dissipation as a function of time. Comparing the gas properties of transitional disks with those for primordial disks will more definitively determine how transitional disks are clearing their gas, complementing studies of dust clearing investigated in our work.

The anonymous referee provided comments and suggestions that greatly improved the organization and content of this paper. We thank Cornelis Dullemond, Thomas Henning, Scott Kenyon, James Muzerolle, Carol Grady, Adam Kraus, and Meredith Hughes for useful conversations and suggestions. We also thank Richard Alexander for providing us with output from his disk evolution models presented in Alexander \& Armitage (2009). Finally, we thank Jeroen Bouwman for assistance with our IRS data reduction. T.C. is supported by a NASA Postdoctoral Fellowship; A.S-A. acknowledges support from the Deutsche Forschungsgemeinschaft (DFG) grant SI-1486/1-1. This work made extensive use of the SIMBAD Astronomical Database.

\section{APPENDIX}

\section{IDENTIFYING TRANSITIONAL DISKS}

\section{A.1. Wavelength Range Required to Meaningfully Constrain Disk Masses}

To determine the wavelength range necessary to meaningfully constrain disk masses, we follow Robitaille et al. (2007) and display SED modeling results for AA Tau, a "typical" optically thick primordial disk (Figure 20). For modeling, we assume an extinction range of $A_{V}=1.45-2.45$ - the average between the Furlan et al. (2006) and Rebull et al. (2010) estimates $\pm 0.5 \mathrm{mag}$ - and use the optical, 2MASS, Spitzer-IRAC/MIPS, and submillimeter data as listed by Robitaille et al. (2007). We adopt the same photometric errors as well.

The best-fit SED including all these data (top-left panel) reproduces the Robitaille et al. results. Our distribution of masses (top right) is sharply peaked about $M_{\text {disk }}=0.015 M_{\odot}$ with a median value of $\sim 0.02 M_{\odot}$, perfectly overlapping with their results. Now we remove longer wavelength data to see if the predicted range of disk masses changes. Restricting ourselves to data equal to or shortward of $70 \mu \mathrm{m}$, the dispersion in disk masses is larger, but clearly and sharply peaked about $M_{\text {disk }} \sim 0.015 M_{\odot} \quad$ (bottom left) with a median value of $\sim 0.01 M_{\odot}$. Modeling optical through MIPS 24 data yields a peak noticeably shifted toward slightly lower disk masses $(\sim 8 \times$ $\left.10^{-3} M_{\odot}\right)$. More generally, the distribution of disk masses traces the shape of the distribution for all possible models (light gray shaded region).

While submillimeter data are required to yield the most precise disk mass estimates, this exercise indicates that farIR wavelengths effectively probe the bulk disk mass. Disk mass estimates derived from modeling optical through far-IR data (e.g., $70 \mu \mathrm{m})$ show good agreement with modeling that 




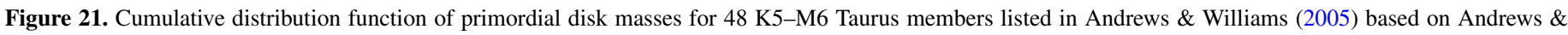

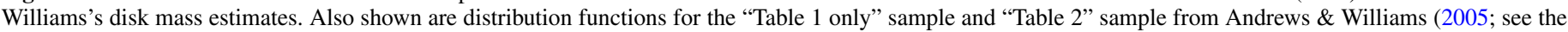

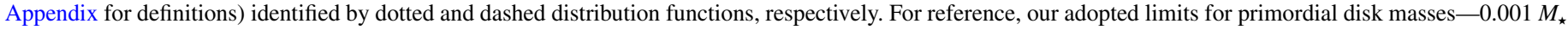
and $0.003 M_{\star}$ - are shown as vertical solid and dotted gray lines, respectively.

includes submillimeter data. Therefore, we conclude that a submillimeter detection is preferred but not required to estimate disk masses with a precision useful for our study. On the other hand, if far-IR/submillimeter data are not available, disk mass estimates are very imprecise and likely not useful for our study.

\section{A.2. Masses of Primordial Disks and Transitional Disks}

SED modeling of optical-to-submillimeter data for stars in 1-2 Myr old clusters like Taurus provides useful benchmark values for disk properties that can be utilized to assess disk evolution. For instance, comparing near-to-mid-IR emission from disks to the median and upper/lower-quartile Taurus SED is often used to identify sources showing evidence for warm dust depletion and/or settling in the inner disk (cf. Hartmann et al. 2005; Furlan et al. 2006; Currie et al. 2009). Similarly, the median and upper/lower quartile of masses for optically thick primordial disks compared to stellar masses as inferred from submillimeter measurements ("fractional disk masses") can be used to assess dust depletion in the outer disk, which traces the total mass of dust (Beckwith \& Sargent 1991; Wood et al. 2002; Andrews \& Williams 2005). Submillimeter data are particularly important in this paper since identifying homologously depleted transitional disks - those with low disk masses compared to primordial disks - presupposes that we can identify appropriate lower limits for primordial disk masses. In this section, we revisit and expand upon the study of Andrews \& Williams (2005) to identify the range of fractional disk masses for primordial disks, showing that the appropriate lower limit is $M_{\text {disk }}=$ $0.001-0.003 M_{\star}$.

The Andrews \& Williams (2005) study includes data for 153 Taurus members (their Table 1). Andrews \& Williams (2005) estimated disk masses for 44 of the members from SED modeling (their Table 2), since they have submillimeter detections and high-quality near-to-far IR photometry: we hereafter refer to this sample as the "Table 2 sample." Disk masses for the other 109 members were estimated solely from their submillimeter fluxes/upper limits: we hereafter refer to this sample as the "Table 1 only sample."

To determine fractional disk masses for disks in both samples, we adopt spectral types from Luhman et al. (2010), use our stellar-mass estimates, and restrict the samples to K5-M6 stars. Because our goal is identifying the masses of optically thick primordial disks, we further trim the sample of Class I protostars, Class III diskless stars, known transitional disks with inner holes (e.g., GM Aur and UX Tau), and other disks with optically thin near-to-mid-IR emission listed in Table 6 (e.g., V836 Tau and JH 223). Finally, we remove foreground nonmembers (St 34) and other stars lacking MIPS data reported in Luhman et al. (2010) and Rebull et al. (2010) that is necessary for SED modeling (e.g., CIDA-11, CIDA-12, and FV Tau/c).

Our selection criteria leave 22 stars in the Table 1 only sample and 26 in the Table 2 sample. The sample of stars that were not modeled by Andrews \& Williams (2005), and thus listed in Table 2, is dominated by Class III diskless members. This characteristic explains the precipitous drop in the number of Table 1 only stars analyzed (22) compared to the nominal sample (109).

Figure 21 shows the cumulative distribution functions for both samples, adopting Andrews \& Williams (2005) disk mass estimates and our stellar-mass estimates. For the Table 2, the median and mean fractional disk masses are $M_{\text {disk }} \sim$ $0.011 M_{\star}$ and $M_{\text {disk }} \sim 0.039 M_{\star}$. The interquartile range covers $0.003-0.033 M_{\star}$. About $88 \%(23 / 26)$ have masses greater than or equal to $0.001 M_{\star}$ : the low-mass outliers are DF Tau, HN Tau, and V955 Tau. For the Table 1 only sample, about $82 \%$ $(18 / 22)$ have masses greater than or equal to $0.001 M_{\star}$ : the low-mass outliers are CIDA-3, CoKu Tau/3, CZ Tau, and DP Tau. To more definitively determine whether any member in either sample has a low-mass disk, we fit their SEDs using the Robitaille et al. (2007) model grid and data reported from Spitzer (Luhman et al. 2010; Rebull et al. 2010) and groundbased studies, following the methods described in Section 4 of the main text. 



Figure 22. SED modeling results for Class II objects with optically thick near-to-mid-IR emission (primordial disks) in Taurus studied in Andrews \& Williams (2005) that have $M_{\text {disk }}<0.001 M_{\star}$ based on the Andrews \& Williams (2005) SED modeling: DF Tau, HN Tau, and V955 Tau. The division corresponding to $M_{\text {disk }} / M_{\star}=$ $10^{(-3)}$ for DF Tau, HN Tau, V955 Tau occurs at $M_{\text {disk }}=1 \times 10^{-3}, 8.5 \times 10^{-4}$, and $5.5 \times 10^{-4}$, respectively. Using the Robitaille models, we find that all of these sources have disk masses larger than $0.001 M_{\star}$

As listed in Table 12 and shown by Figure 22, inferred disk masses for DF Tau, HN Tau, and V955 Tau using the Robitaille models are substantially higher than values listed by Andrews \& Williams (2005). The median masses of the best-fit models are (5-9) $\times 10^{-3} M_{\star}$, exceeding both the $0.001 M_{\star}$ limit and the previously quoted lower-quartile fractional disk mass. Thus, we conclude that all of the optically thick primordial disks modeled by Andrews \& Williams (2005) have inferred masses greater than $M_{\text {disk }}=0.001 M_{\star}$.

We obtain similar results for the low-mass outliers in our second sample. Figure 23 shows the SEDs and distribution of masses from best-fit Robitaille models for CIDA-3, CoKu Tau/ 3, CZ Tau, and DP Tau. CoKu Tau/3 and DP Tau have inferred masses greater than $0.001 M_{\star}$. CZ Tau and CIDA-3 have much 

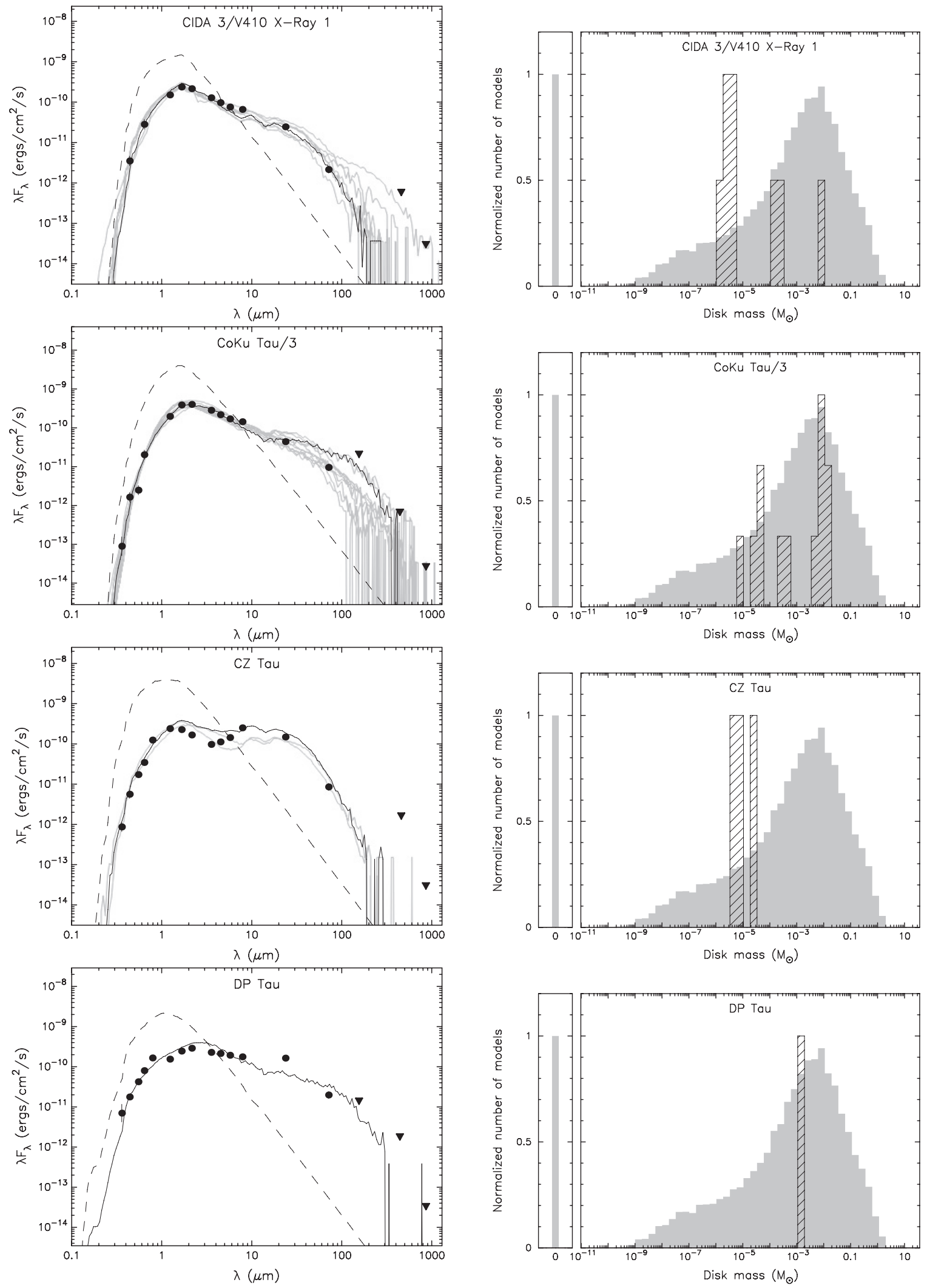

Figure 23. SED fits and distribution of masses from best-fitting models for other primordial disks in Taurus with data from Andrews \& Williams (2005). The division corresponding to $M_{\text {disk }} / M_{\star}=10^{(-3)}$ for CIDA-3/V410 X-Ray 1 , CoKu Tau/3, CZ Tau, and DP Tau occurs at $M_{\text {disk }}=6 \times 10^{-4}, 6 \times 10^{-4}, 5.5 \times 10^{-4}$, and $4 \times$ $10^{-4}$, respectively. Based on the Andrews \& Williams (2005) submillimeter estimates, all of these have disk masses less than $0.001 M_{\star}$. Our analysis finds that only two of the four have such low masses. 


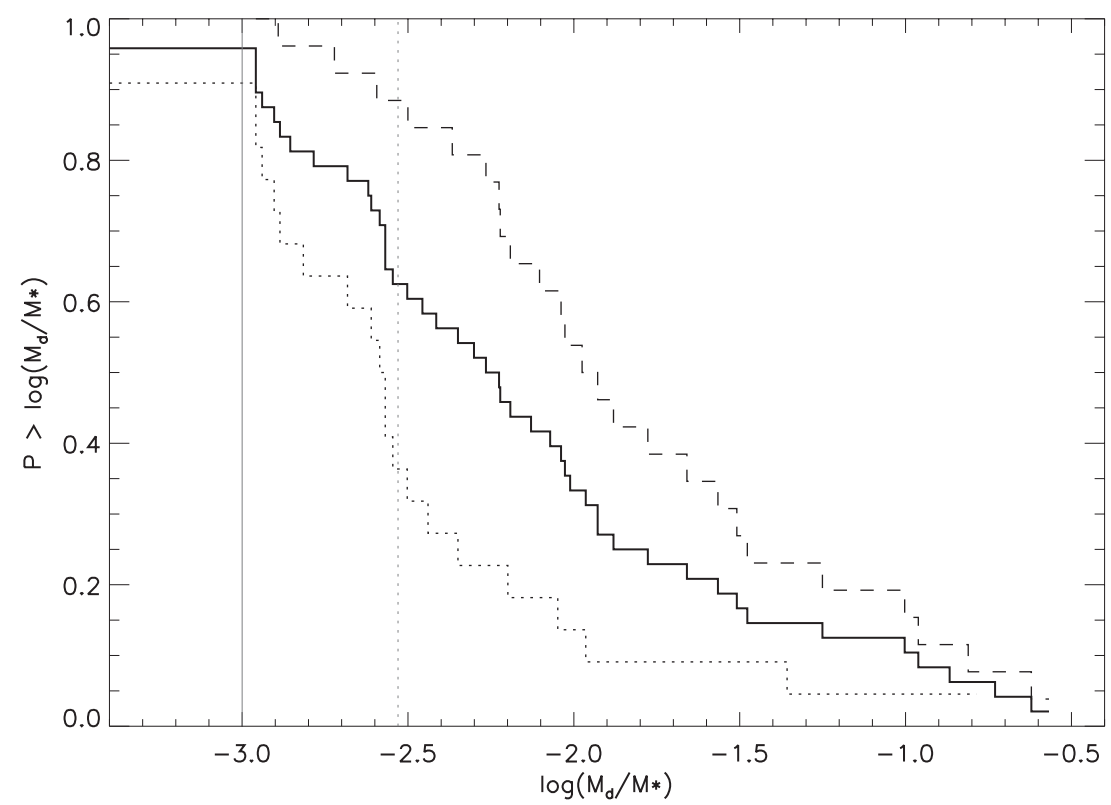

Figure 24. Cumulative distribution function of primordial disk masses for 48 K5-M6 Taurus members listed in Andrews \& Williams (2005) taking into account revised mass estimates for Taurus members discussed in the Appendix. Our results show that the disk mass range of $0.001-0.003 M_{\star}$ defines a meaningful lower limit for the masses of optically thick primordial disks.

Table 12

Optically Thick Primordial Disks Around K5-M6 Stars in Taurus Listed as having Low Masses in Andrews \& Williams (2005)

\begin{tabular}{lcrrrrr}
\hline \hline \multicolumn{1}{c}{ Name } & ST & $M_{\star}$ & $M_{\text {disk }}\left(M_{\odot}\right)($ AW05 $)$ & $M_{\text {disk }} / M_{\star}(\text { AW05 })^{\mathrm{a}}$ & $M_{\text {disk }}\left(M_{\odot}\right.$, This Work $)$ & $M_{\text {disk }} / M_{\star}($ This Work $)$ \\
\hline HN Tau & K5 & 1.0 & $8 \times 10^{-4}$ & $8 \times 10^{-4}$ & $6 \times 10^{-3}$ & $6 \times 10^{-3}$ \\
V955 Tau & K7 & 0.85 & $5 \times 10^{-4}$ & $5.9 \times 10^{-4}$ & $4 \times 10^{-3}$ & $5 \times 10^{-3}$ \\
DF Tau & M2 & 0.55 & $4 \times 10^{-4}$ & $7.3 \times 10^{-4}$ & $5 \times 10^{-3}$ & $9 \times 10^{-3}$ \\
CoKu Tau/3 & M1 & 0.6 & $<4 \times 10^{-4}$ & $<6.7 \times 10^{-4}$ & $5 \times 10^{-3}$ & $8 \times 10^{-3}$ \\
DP Tau & M1 & 0.6 & $<5 \times 10^{-4}$ & $<8.3 \times 10^{-4}$ & $2 \times 10^{-3}$ & $3 \times 10^{-3}$ \\
CIDA-3/V410 X-Ray 1 & M2 & 0.55 & $<4 \times 10^{-4}$ & $<7.3 \times 10^{-4}$ & $2 \times 10^{-5}$ & $4 \times 10^{-5}$ \\
CZ Tau & M3 & 0.4 & $<4 \times 10^{-4}$ & $<10^{-3}$ & $2 \times 10^{-5}$ & $5 \times 10^{-5}$ \\
\hline
\end{tabular}

Note. ${ }^{a}$ Fractional disk mass is derived using our estimates for stellar mass.

lower masses and represent the only optically thick primordial disks from the Andrews \& Williams (2005) sample with inferred masses lower than $0.001 M_{\star}$.

Figure 24 shows the revised cumulative distribution functions for the fractional masses of optically thick primordial disks surrounding K5-M6 stars in Taurus. Except for the sources individually modeled in this section (listed in Table 12), we adopt disk mass values from Andrews \& Williams (2005) for simplicity. Of these members, $\sim 96 \%(46 / 48)$ have masses greater than or equal to $0.001 M_{\star}$. The median and interquartile range for the Table 2 sample is $0.011 M_{\star}$ and $0.006-0.033 M_{\star}$. For the combined sample of 48 stars, these values are $5 \times$ $10^{-3} M_{\star}$ and $0.0024-0.015 M_{\star}$; however, most of the masses are derived from submillimeter fluxes alone, so individual mass estimates accounting for the entire disk should be larger and the median and quartile disk masses should likewise be larger.

Since nearly all primordial disks in the Andrews \& Williams (2005) sample have masses greater than $0.001 M_{\star}$ and the lowerquartile disk mass for the modeled sample is $>0.003 M_{\text {star }}$, we conclude that disks with masses lying below $0.001-0.003 M_{\star}$ lie below the range expected for primordial disks. Thus, based on modeling current optical through submillimeter data, defining the lower-mass limit for optically thick primordial disks at $0.001-0.003 M_{\star}$ is justified.

\section{REFERENCES}

Adams, F., Lada, C. J., \& Shu, F. 1987, ApJ, 312, 788

Alexander, R., \& Armitage, P. 2009, ApJ, 704, 989

Andre, P., \& Montmerle, T. 1994, ApJ, 420, 837

Andrews, S., \& Williams, J. 2005, ApJ, 631, 1134

Andrews, S., \& Williams, J. 2007, ApJ, 671, 1800

Audard, M., et al. 2007, A\&A, 468, 379

Backman, D., \& Paresce, F. 1993, in Protostars and Planets III, ed. E. H. Levy \& J. I. Lunine (Tucson, AZ: Univ. Arizona Press), 1253

Baraffe, I. 1998, A\&A, 337, 403

Beckwith, S., \& Sargent, A. 1991, AJ, 381, 250

Beckwith, S., et al. 1990, AJ, 99, 924

Bouwman, J., et al. 2006, ApJ, 653, 57

Bouwman, J., et al. 2008, ApJ, 683, 479

Bromley, B., \& Kenyon, S. J. 2011, ApJ, submitted (arXiv:1012.0574)

Calvet, N., et al. 2002, ApJ, 568, 1008

Calvet, N., et al. 2005, ApJ, 630, 185L

Carmona, A., et al. 2007, A\&A, 464, 687

Carpenter, J., et al. 2009, ApJ, 705, 1646

Casey, B., et al. 1998, AJ, 115, 1617

Chen, C., et al. 2005, ApJ, 623, 493

Chiang, E., \& Goldreich, P. 1997, ApJ, 490, 368

Chiang, E., \& Goldreich, P. 1999, ApJ, 519, 279

Chini, R., et al. 2003, A\&A, 409, 235

Cieza, L., et al. 2008, ApJ, 686, 115L

Cieza, L., et al. 2009, ApJ, 696, 84L

Cieza, L., et al. 2010, ApJ, 712, 925

Currie, T. 2010, arXiv:1002.1715

Currie, T., \& Kenyon, S. J. 2009, AJ, 138, 703 
Currie, T., et al. 2007, ApJ, 659, 599

Currie, T., et al. 2008, ApJ, 672, 558

Currie, T., et al. 2009, ApJ, 698, 1

Currie, T., et al. 2010, ApJS, 186, 131

Dahm, S. 2005, AJ, 130, 1805

Dahm, S. 2008, AJ, 136, 521

Dahm, S., \& Hillenbrand, L. 2007, AJ, 133, 2072

D’Alessio, P., et al. 2006, ApJ, 638, 314

Ercolano, B., Clarke, C., \& Robitaille, T. 2009, MNRAS, 394, L141

Espaillat, C. 2009, $\mathrm{PhD}$ thesis, Univ. Michigan

Espaillat, C., et al. 2007, ApJ, 670, L135

Espaillat, C., et al. 2011, ApJ, 728, 49

Evans, N., et al. 2009, ApJS, 181, 321

Fedele, D., et al. 2010, A\&A, 510, 72

Flaherty, K., et al. 2007, AJ, 663, 1069

Forbrich, J., \& Preibisch, T. 2007, A\&A, 475, 959

Furlan, E., et al. 2006, ApJS, 165, 568

Gautier, T., et al. 2008, ApJ, 683, 813

Groppi, C., et al. 2007, ApJ, 670, 489

Gustafsson, B., et al. 2008, A\&A, 486, 951

Gutermuth, R., et al. 2008, ApJ, 674, 336

Hartmann, L., et al. 2005, ApJ, 629, 881

Hartmann, L., et al. 2006, ApJ, 648, 484

Henning, Th., \& Stognienko, R. 1996, A\&A, 311, 291

Henning, Th., et al. 1994, A\&A, 291, 546

Herczeg, G., \& Hillenbrand, L. 2008, ApJ, 681, 594

Hernandez, J., et al. 2007a, ApJ, 662, 1067

Hernandez, J., et al. 2007b, ApJ, 671, 1784

Higdon, S., et al. 2004, PASP, 116, 975

Houck, J. R., et al. 2004, ApJS, 154, 18

Hughes, A. M., et al. 2008, ApJ, 698, 131

Hughes, A. M., et al. 2010, AJ, 140, 887

Indebetouw, R., et al. 2005, ApJ, 619, 931

Ireland, M., \& Kraus, A. 2008, ApJ, 678, L59

Irwin, J., et al. 2008, MNRAS, 384, 675

Jensen, E., et al. 1994, ApJ, 429, L29

Jewitt, D. 1994, AJ, 108, 661

Kennedy, G., \& Kenyon, S. J. 2009, ApJ, 695, 1210

Kenyon, S. J., \& Bromley, B. 2008, ApJS, 179, 451

Kenyon, S. J., \& Bromley, B. 2009, ApJ, 690, L140

Kenyon, S. J., \& Hartmann, L. 1987, ApJ, 323, 714

Kenyon, S. J., \& Hartmann, L. 1995, ApJS, 101, 117

Kenyon, S. J., et al. 2008, in Handbook of Starforming Regions, Vol. 1: The Northern Sky, ed. B. Reipurth (ASP Monograph Publ. Vol. 4; San Francisco, CA: ASP), 405

Kraus, A., et al. 2006, ApJ, 649, 306

Kraus, A., et al. 2011, ApJ, in press (arXiv:1101.4016)

Lada, C. J., et al. 2006, AJ, 131, 1574

Lawson, W., Lyo, A-R., \& Muzerolle, J. 2004, MNRAS, 351, L39
Lopez-Marti, B., et al. 2005, A\&A, 444, 175

Lopez-Marti., B., et al. 2010, A\&A, 515, 31

Luhman, K., \& Steeghs, D. 2004, ApJ, 609, 917

Luhman, K., et al. 2003, ApJ, 593, 1093

Luhman, K., et al. 2009, ApJ, 703, 399

Luhman, K., et al. 2010, ApJS, 186, 111

Lyo, A-R., et al. 2004, MNRAS, 355, 363

Marraco, H., \& Rydgren, A. 1981, AJ, 86, 62

Merin, B., et al. 2010, ApJ, 718, 1200

Meyer, M., \& Wilking, B. 2009, PASP, 121, 350

Moitinho, A., et al. 2001, ApJ, 563, 73

Muench, A., et al. 2007, AJ, 134, 411

Mulders, G. D., Dominik, C., \& Min, M. 2010, A\&A, 510, 11

Muzerolle, J. 2010, HiA, 15, 732

Muzerolle, J., et al. 2010, ApJ, 708, 1107

Neuhauser, R., \& Forbrich, J. 2008, in Handbook of Starforming Regions, Vol.

2: The Southern Sky, ed. B. Reipurth (ASP Monograph Publ. Vol. 5; San Francisco, CA: ASP), 735

Nisini, B., et al. 2005, A\&A, 429, 543

Pascucci, I., \& Sterzik, M. 2009, ApJ, 702, 724

Patten, B. M. 1998, PASP, 154, 1755

Plavchan, P., et al. 2009, ApJ, 698, 1068

Ramsay-Howat, S., \& Greaves, J. 2007, MNRAS, 379, 1658

Rebull, L., et al. 2010, ApJS, 186, 259

Rieke, G. H., et al. 2005, ApJ, 620, 1010

Robitaille, T., et al. 2006, ApJS, 167, 256

Robitaille, T., et al. 2007, ApJS, 169, 328

Salyk, C., et al. 2009, ApJ, 699, 330

Sicilia-Aguilar, A., Henning, Th., \& Hartmann, L. 2010, ApJ, 710, 597

Sicilia-Aguilar, A., et al. 2005, AJ, 130, 188

Sicilia-Aguilar, A., et al. 2006a, AJ, 132, 2135

Sicilia-Aguilar, A., et al. 2006b, ApJ, 638, 897

Sicilia-Aguilar, A., et al. 2007, ApJ, 659, 1637

Sicilia-Aguilar, A., et al. 2008, ApJ, 687, 1145

Sicilia-Aguilar, A., et al. 2009, ApJ, 701, 1188

Simon, M., \& Prato, L. 1995, ApJ, 450, 824

Skrutskie, M. 1990, AJ, 99, 1187

Skrutskie, M., et al. 2006, AJ, 131, 1163

Strom, K., et al. 1989, AJ, 97, 1451

Thalmann, C., et al. 2010, ApJ, 718, 87L

Torres, C., et al. 2006, A\&A, 460, 695

White, R. J., \& Ghez, A. M. 2001, ApJ, 556, 265

White, R. J., \& Hillenbrand, L. A. 2004, ApJ, 616, 998

Whitney, B., et al. 2003a, ApJ, 591, 1049

Whitney, B., et al. 2003b, ApJ, 598, 1079

Wilking, B., et al. 1985, ApJ, 293, 165

Wilking, B., et al. 1992, ApJ, 397, 520

Willner, S., et al. 1982, ApJ, 253, 174

Wolk, S. J., \& Walter, F. 1996, AJ, 111, 2066

Wood, K., et al. 2002, ApJ, 567, 1183 